\title{
Conformal Mapping in Linear Time
}

\author{
Christopher J. Bishop
}

Received: 29 April 2008 / Revised: 19 March 2010 / Accepted: 13 May 2010 /

Published online: 15 June 2010

(C) Springer Science+Business Media, LLC 2010

\begin{abstract}
Given any $\epsilon>0$ and any planar region $\Omega$ bounded by a simple $n$-gon $P$ we construct a $(1+\epsilon)$-quasiconformal map between $\Omega$ and the unit disk in time $C(\epsilon) n$. One can take $C(\epsilon)=C+C \log \frac{1}{\epsilon} \log \log \frac{1}{\epsilon}$.

Keywords Numerical conformal mappings · Schwarz-Christoffel formula · Hyperbolic 3-manifolds · Sullivan's theorem · Convex hulls · Quasiconformal mappings · Quasisymmetric mappings · Medial axis · CRDT algorithm
\end{abstract}

\section{Introduction}

If $\Omega$ is a proper, simply connected plane domain, then by the Riemann mapping theorem there is a conformal map $f: \mathbb{D} \rightarrow \Omega$, but for most domains there is no simple, explicit formula. In this paper, we will show that there is "almost" such a formula in the sense that there is a linear time algorithm for computing the conformal map with estimates on time and accuracy that are independent of the geometry of the particular domain. Thus the computational complexity of conformal mapping is linear in the following sense.

Theorem 1 Given a simply connected domain $\Omega$ bounded by an $n$-gon we can compute the conformal map $f: \mathbb{D} \rightarrow \Omega$ to within quasiconformal error $\epsilon$ in time $O(n \cdot p \log p)$ where $p=O\left(\log \frac{1}{\epsilon}\right)$.

The phrases "can compute" and "quasiconformal error" require some explanation in order to make this a precise mathematical statement. A unit of work consists of an infinite precision arithmetic operation or an evaluation of exp or log.

The author is partially supported by NSG Grant DMS 10-06309.

C.J. Bishop $(\bowtie)$

Mathematics Department, SUNY at Stony Brook, Stony Brook, NY 11794-3651, USA

e-mail: bishop@math.sunysb.edu 
We will cover the unit disk by $O(n)$ regions (disks and annuli) and in each region approximate the conformal map using a $p$-term power or Laurent series and some elementary functions. Combining these using a partition of unity will give a $(1+\epsilon)$ quasiconformal map from $\mathbb{D}$ to $\Omega$. Our series converge geometrically fast on the associated regions, and so each series has $p \sim \log \frac{1}{\epsilon}$ terms in general. The fastest known methods for multiplication, division, composition or inversion of power series use the fast Fourier transform, and the time to perform an FFT on a $p$-term power series is $\operatorname{FFT}(p)=O(p \log p)$, so Theorem 1 says we only need $O(1)$ such operations per vertex.

The Schwarz-Christoffel formula (see Appendix A) provides a formula for the conformal map onto a polygon, but involves unknown parameters (the conformal preimages of the vertices). Thus, it is not really a solution of the mapping problem, but simply reduces it to finding the $n$ conformal prevertices. Suppose $\Omega$ is bounded by a simple $n$-gon with vertices $\mathbf{v}=\left\{v_{1}, \ldots, v_{n}\right\}$, let $f: \mathbb{D} \rightarrow \Omega$ be conformal and let $\mathbf{z}=f^{-1}(\mathbf{v})$ be the conformal prevertices. A more concrete version of Theorem 1 is:

Theorem 2 Given any $\epsilon>0$ there is a $C=C(\epsilon)<\infty$ so that if $\Omega$ is bounded by a simply polygon $P$ with $n$ vertices we can find points $\mathbf{w}=\left\{w_{1}, \ldots, w_{n}\right\} \subset \mathbb{T}$ so that

(1) All $n$ points in $\mathbf{w}$ can be computed in at most $C n$ steps.

(2) $d_{Q C}(\mathbf{w}, \mathbf{z})<\epsilon$ where $\mathbf{z}$ are the true conformal prevertices.

Here $d_{Q C}(\mathbf{w}, \mathbf{z})=\inf \{\log K: \exists K$-quasiconformal $h: \mathbb{D} \rightarrow \mathbb{D}$ such that $h(\mathbf{z})=\mathbf{w}\}$. The constant $C(\epsilon)$ may be taken to be $C+C \log \frac{1}{\epsilon} \cdot \log \log \frac{1}{\epsilon}$ where $C$ is independent of $\epsilon$ or $n$.

Note that $d_{Q C}(\mathbf{w}, \mathbf{z})=0$ iff the $n$-tuples are Möbius images of each other. It is not hard to see that this happens iff the corresponding polygons are linear images of each other, and so this is a natural metric for the problem. Quasiconformal approximation implies uniform approximation but is stronger; not only are the points of $\mathbf{w}$ within $O(\epsilon)$ of the corresponding points of $\mathbf{z}$, but the relative arrangement of $\mathbf{w}$ approximates the corresponding arrangement of $\mathbf{z}$ equally well at every scale (see Lemma 45).

We will define a quadratically convergent iteration on $n$-tuples in $\mathbb{T}$ and provide a starting point from which it is guaranteed to converge with an estimate independent of the domain. Although there are various details to check, each of basic ideas involved is fairly easy to explain and involves a geometric construction. We will discuss these briefly here, leaving the details and difficult cases for the rest of the paper.

The first idea is to consider the so called "iota-map", $\iota: P \rightarrow \mathbb{T}$, to obtain an $n$-tuple $\mathbf{w}=\iota(\mathbf{v}) \subset \mathbb{T}$ that is only a bounded $d_{Q C}$-distance $K$ from the true prevertices (it is known from [14] that we can take $K \leq 7.82$ ). The definition of this map and the proof that it has the desired approximation properties are motivated by results from hyperbolic 3-dimensional geometry, but we can give a simple, geometric description in the plane. We approximate our polygon by a finite collection of medial axis disks (these are subdisks of the domain whose boundary hits the boundary of the domain in at least two points). The union of these disks, $\Omega$, can be written as a union of a single $D$ disk and a collection of disjoint crescents; see Fig. 1. Each crescent is 

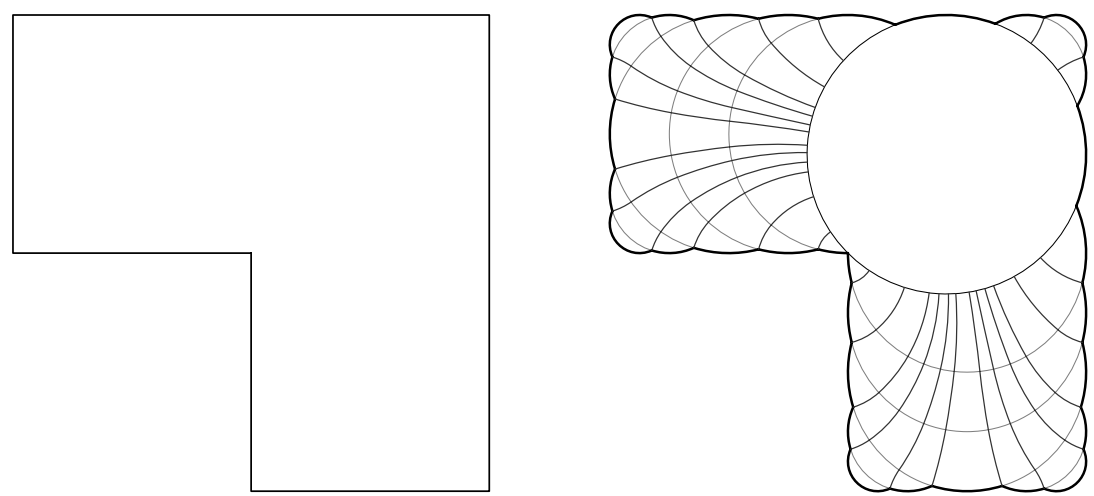

Fig. 1 An example where we have approximated a domain by a union of disks; written the new domain $\Omega$ as a disjoint union of one disk $D$ and several crescents; and used circular arcs orthogonal to the crescents to define a flow from $\partial \Omega$ to $\partial D$. The resulting map is close to the Riemann map with estimates independent of the domain
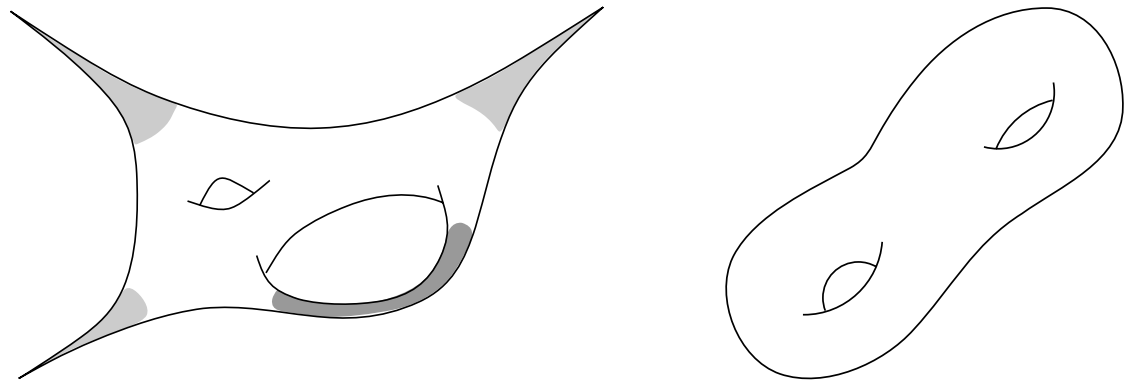

Fig. 2 On the left is a surface with one hyperbolic thin part (darker) and three parabolic thin parts (lighter). On the right is a "thick" surface with no thin parts

foliated by circular arcs orthogonal to its two boundary arcs. Following leaves of this foliation gives the desired map $\iota: \partial \Omega \rightarrow \partial D$. The initial approximation by a union of disks is unnecessary, but convenient for various reasons (the $\iota$ map for a polygon can be computed directly, using the medial axis of the polygon; see, e.g., [17]). The construction of $\iota$ in linear time depends on the fact that the medial axis of a $n$-gon can be computed in linear time, a result of Chin, Snoeyink and Wang [33].

The next idea is to decompose polygons into pieces, again following a motivation from hyperbolic geometry. A standard technique in the theory of hyperbolic manifolds is to partition the manifold into its thick and thin parts (based on the length of the shortest non-trivial loop through each point); see Fig. 2. Thin parts often cause technical difficulties, but this is partially compensated for by the fact that there are only a few possible types of thin parts and each has a well understood shape. Thus we can think of the manifold as consisting of some "interesting" thick parts attached to some annoying, but explicitly described, thin parts. The manifold is considered especially nice if it is thick, i.e., no thin parts occur. 
Fig. 3 A polygon with one hyperbolic thin part (darker) and six parabolic thin parts, which we further divide into two groups corresponding to interior vertex angles $<\pi$ and $>\pi$
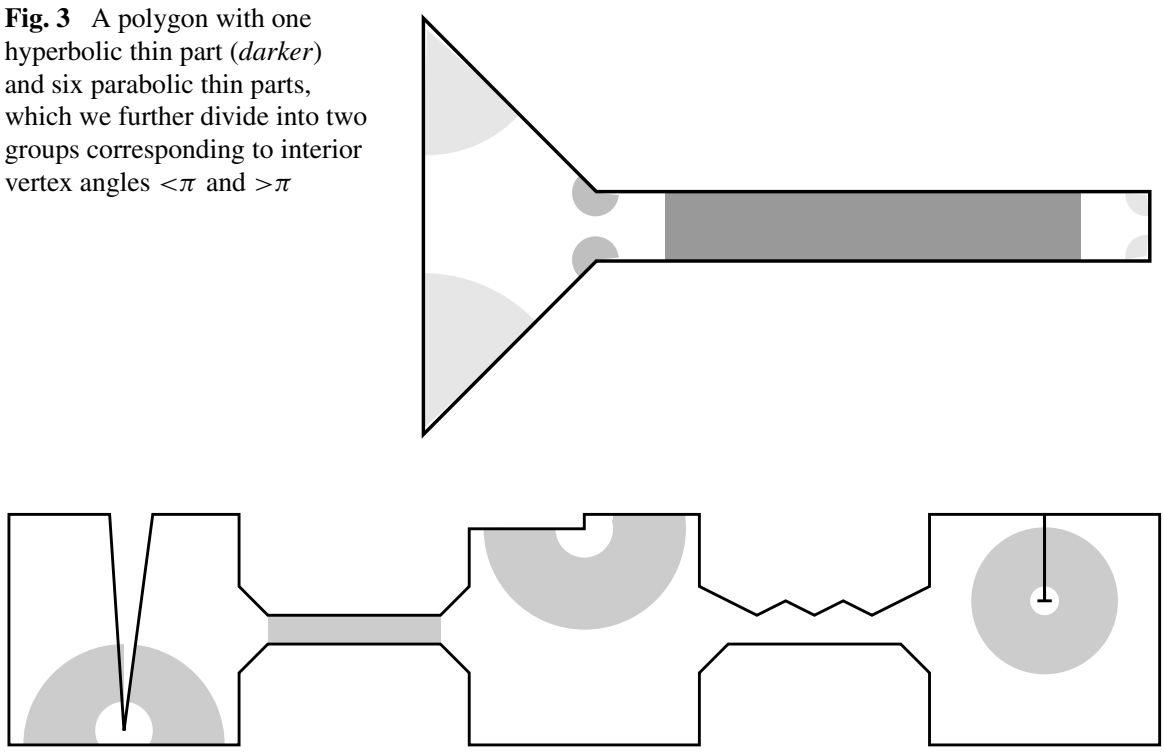

Fig. 4 The five hyperbolic thin parts of this polygon are shaded gray. The channel on the right is not thin because there are many vertices lining one side of it. The complementary white regions are "thick"; one of our strategies is to compute mappings onto thick domains and "glue" them together across the thin connecting regions

We will describe an analogous decomposition of a polygon into thick and thin parts. The thin parts occur when the extremal length between two edges is very small (roughly this means the Euclidean distance inside the domain between the edges is small compared to their Euclidean diameters). This occurs whenever the edges are adjacent, but we shall be mostly interested in thin parts corresponding to non-adjacent edges and we denote the two cases as parabolic and hyperbolic, respectively, in analogy to the thin parts of a Riemann surface (in that case, parabolic thin parts are noncompact and have one boundary component attached to the thick part of the surface; hyperbolic thin parts are compact and have two boundary components, both attaching to the thick part of the surface); see Fig. 3. The parabolic thin parts look like sectors, and the hyperbolic thin parts look like generalized quadrilaterals (with two sides on the boundary of the given polygon). We say the polygon is thick if no hyperbolic thin parts occur; see Fig. 4 for various ways hyperbolic thin parts can arise.

As with manifolds, the thin parts of polygons cause technical difficulties. However, our thin parts can only have a small number of simple shapes and the conformal maps from the disk into a thin part can be well approximated by explicit formulas. Thus they are "well understood". Indeed, much of the algorithm described in this paper will only be applied to the remaining thick parts, making them the "interesting" part of the polygon. Thus, as with hyperbolic manifolds, polygons will be divided into interesting thick parts, attached to annoying, but well understood, thin parts.

The next idea concerns how to represent a map onto $\Omega$. A conformal map onto a polygon has a convergent power series on $\mathbb{D}$, but since $f^{\prime}$ is discontinuous at the prevertices, it converges slowly and the number of terms needed for a given accuracy 


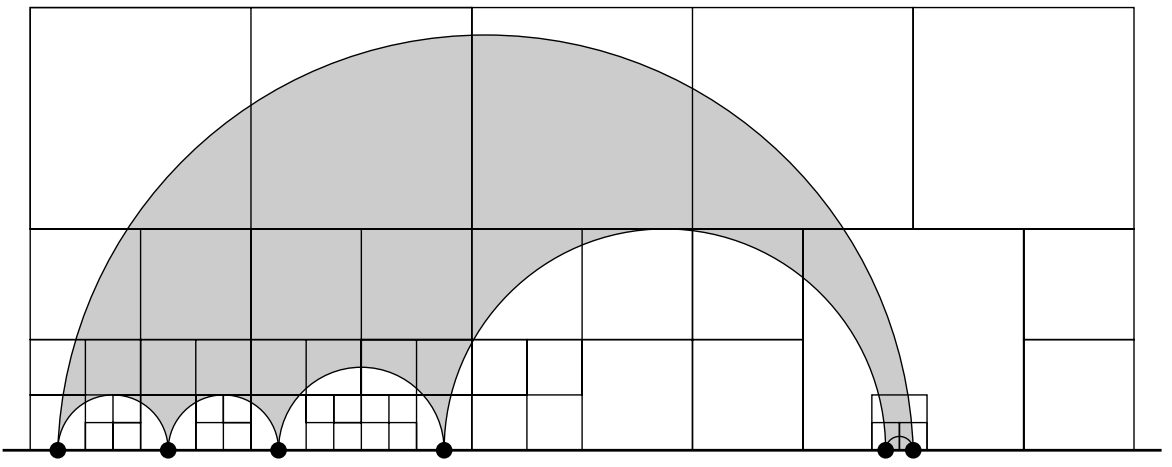

Fig. 5 Decompose the plane by first covering the convex hull of the prevertices. This picture has one arch on the right hand side. Arches correspond to clusters of two or more prevertices which are isolated in a precise way

depends on the geometry of the image domain. For convenience, we will replace the disk by the upper half-plane $\mathbb{H}$, and we will represent a map $f: \mathbb{H} \rightarrow \Omega$ by breaking $\mathbb{H}$ into $O(n)$ simple pieces and using a $p$-term power series or Laurent expansion on each piece that represents $f$ with error $\leq 2^{-p}$, independent of the geometry of $\Omega$. The series are combined using a partition of unity to give a single quasiconformal map whose dilatation can be computed and corrected for to give an improved approximation. The decomposition $\mathcal{W}$ of $\mathbb{H}$ is accomplished by taking the hyperbolic convex hull of the point set $S$ (our current prevertex approximation) and covering it by $O(n)$ Whitney boxes, Carleson squares and regions we call arches and then dividing the remaining regions, which all lie outside the convex hull, into $O(n)$ Carleson boxes; see Fig. 5.

More precisely, an $\epsilon$-representation of a polygon is a triple $(S, \mathcal{W}, \mathcal{F})$, where $S$ is a $n$-tuple in $\mathbb{R}$ (the prevertices), $\mathcal{W}$ is a decomposition of $\mathbb{H}$ into $O(n)$ simple pieces and $\mathcal{F}$ is a collection of functions, one for each piece of our decomposition. These functions consist of $p=O(|\log \epsilon|)$ terms of a series expansion on each piece, and a choice of a certain elementary function for each piece, which is the identity or a power function in most cases. Moreover, we require that functions for adjacent pieces agree to within $\epsilon$ (in a certain metric) along the common boundary.

We will prove a Newton type iteration for improving $\epsilon$-representations. We will show that there is an absolute constant $\epsilon_{0}>0$ (independent of $n$ and $\Omega$ ) so that if $\epsilon<\epsilon_{0}$, then we can quickly improve a $\epsilon$-representation to a $\epsilon^{2}$-representation. Thus starting with a $n$-tuple at distance $\epsilon_{0}$ from the true answer, it only takes $O\left(\log \log \frac{1}{\epsilon}\right)$ iterations to reach accuracy $\epsilon$. The main problem is to estimate the time needed to perform each iteration.

Combining the functions in $\mathcal{F}$ with a piecewise polynomial partition of unity gives a $(1+\epsilon)$-quasiconformal map from $F: \mathbb{H} \rightarrow \Omega$. Let $\mu=\bar{\partial} F / \partial F$ be the Beltrami dilatation of $F$. Then $\|\mu\|_{\infty}=O(\epsilon)$ and $\mu$ can be explicitly computed from the series expansions in $\mathcal{F}$ and the partition of unity. If we could solve a Beltrami equation to find a mapping $H$ of the upper half-plane to itself so that $\mu_{H}=\mu_{F}$, then $F \circ H^{-1}$ would be the desired conformal map. We can't solve this equation 
exactly in finite time, but we can solve $\mu_{H}=\mu_{F}+O\left(\left\|\mu_{F}\right\|_{\infty}^{2}\right)$ in linear time using the fast multipole method of Greengard and Rokhlin. Thus $F \circ H^{-1}$ will be $\left(1+O\left(\epsilon^{2}\right)\right)$-quasiconformal, and this is the improved representation. Each iteration consists of approximately solving an equation $\bar{\partial} H=\mu$ by evaluating $p=O\left(\log \frac{1}{\epsilon}\right)$ terms of the power series of a Beurling transform of $\mu$ on $n$ disks. Using a fast multipole method and fast manipulation of power series, we can do each iteration in time $O(n p \log p)=O\left(n \log \frac{1}{\epsilon} \cdot \log \log \frac{1}{\epsilon}\right)$. Moreover, since $p=|\log \epsilon|$ increases geometrically with each iteration, the total work is dominated by the final iteration, which gives the desired estimate.

Another basic idea of the paper deals with how to improve our initial $n$-tuple (provided by the $\iota$ map) that is at most distance $K$ from the correct answer to an $n$-tuple that is within the distance $\epsilon_{0}$ required by the Newton type iteration. This is accomplished by connecting our domain to the unit disk by a chain of $N+1=$ $O\left(1 / \epsilon_{0}\right)$ regions $\mathbb{D}=\Omega_{0}, \ldots, \Omega_{N}=\Omega$. As before, it is convenient to work with a domain that is a finite union of disks (such domains are also called "finitely bent" for reasons that will be clear when we discuss the dome of a domain later).

In this case, there is a "normal crescent" decomposition of $\Omega$. If $\Omega=\bigcup D_{k}$ is a finite union of disks and $\partial D_{1} \cap \partial D_{2} \cap \partial \Omega \neq \emptyset$, then the corresponding crescent is the subregion of $\Omega$ bounded by circular arcs perpendicular to $\partial D_{1}$ and $\partial D_{2}$ at their two intersection points. Removing every such crescent from a finitely bent domain leaves a collection of "gaps"; see Fig. 6. This decomposition has a natural interpretation in terms of 3-dimensional hyperbolic geometry. Each planar domain $\Omega$ is associated to a surface $S$ (called the dome of $\Omega$ ) in the upper have space that is the upper envelope of all hemispheres with base disk in $\Omega$. If $\Omega$ is a finite union of disks then the dome is a finite union of geodesic faces that meet along hyperbolic geodesics called the bending lines. There is a map $R: \Omega \rightarrow S$ (the hyperbolic nearest point projection onto $S$ ) and the gaps in $\Omega$ are simply the points that map to faces of $S$ and the crescents are points that map to bending lines.

Given the normal crescent decomposition of the domain, we can build a one parameter family of regions by varying the angles of the crescents (this procedure is called "angle scaling"). When the angles have all been collapsed to zero, the resulting domain is the disk; see Fig. 7 for an example of such a chain. More examples are illustrated in Figs. 23 to 26.

We shall see that each domain in the chain is mapped to the next by an explicit map $g_{k}: \Omega_{k} \rightarrow \Omega_{k+1}$ with small quasiconformal constant. This will allow us to convert an $\epsilon$-representation for one domain in our chain into a $2 \epsilon$-representation for the next one. We can then use our improvement iteration to improve $2 \epsilon$ to $\epsilon$ and repeat the process. In this way, we can start with a representation of the disk (which is easy to find) and finish with one for $\Omega$ (which is what we want), in a uniformly bounded number of steps.

There are (at least) two alternatives for approximating a conformal map $f: \mathbb{D} \rightarrow$ $\Omega$ : approximate it by conformal maps $f_{n}: \mathbb{D} \rightarrow \Omega_{n}$ where $\Omega_{n}$ converges to $\Omega$, or approximate by maps $f_{n}: \mathbb{D} \rightarrow \Omega$ which are not conformal, but converge to the conformal map. The first approach is natural when dealing with Schwarz-Christoffel maps since a choice of parameters defines a conformal map onto a region with the right angles, but perhaps the wrong side lengths. We then adjust the parameters to get 

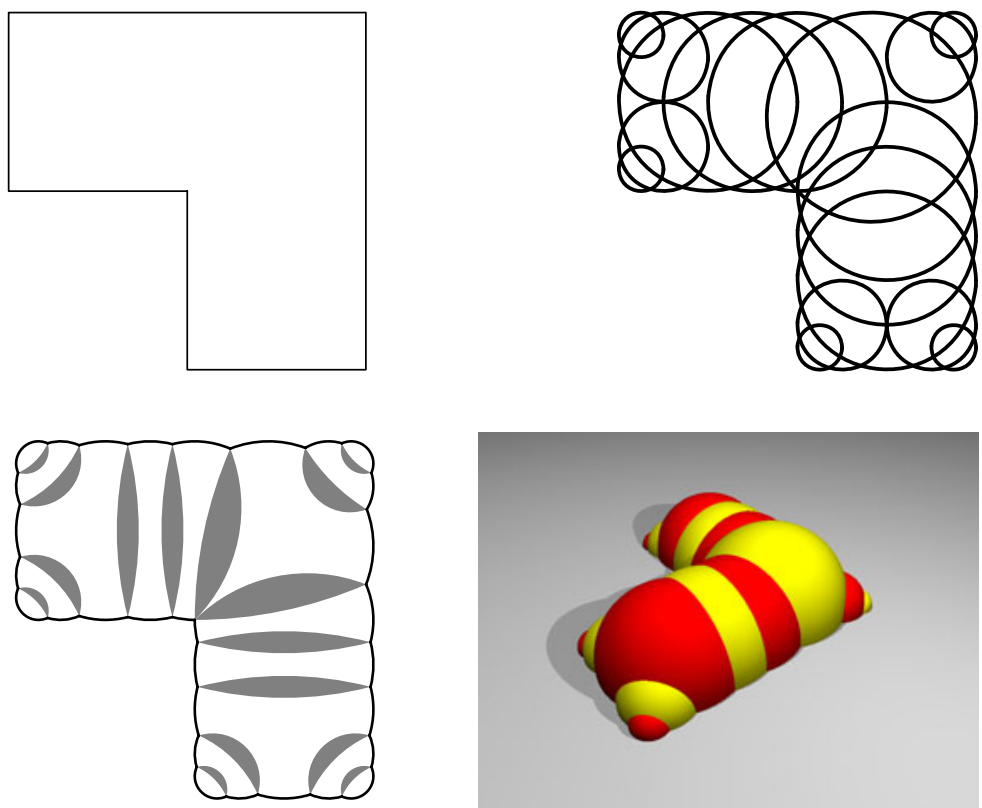

Fig. 6 A polygon, an approximation by a finite union of disks, its normal crescent decomposition and the corresponding dome
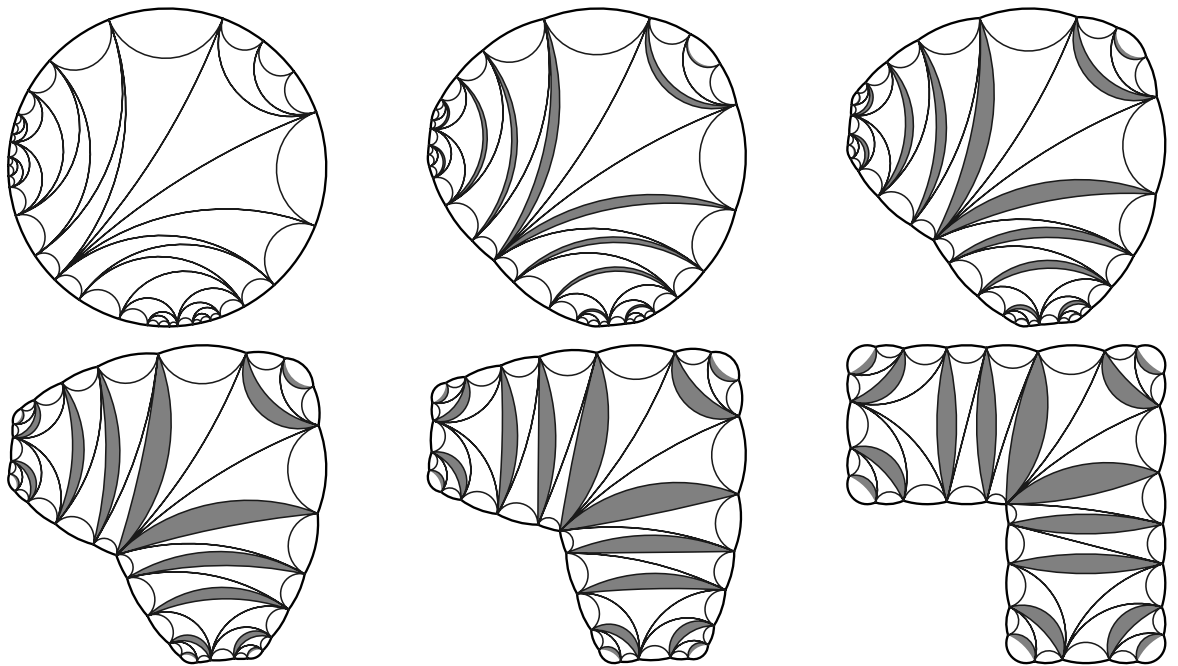

Fig. 7 Deforming the disk to an approximate polygon. The gaps have been subdivided to make our decomposition into a hyperbolic triangulation of the disk

a better approximation to the target domain. There are various heuristics for doing this that work in practice, but the relation between the parameters and the geometry of the image can be subtle and I have not seen how to prove convergence for any 
such method. In this paper, I take the second choice above. Information about the geometry of $\Omega$ is built directly into our approximating functions, and our iteration merely has to force the approximation to be "more conformal"; this can be done without reference to $\Omega$, and hence with estimates independent of $\Omega$. This choice also leads naturally to the representation of these maps using power series on WhitneyCarleson decompositions to enforce the desired boundary conditions.

The $\epsilon$-representations used to approximate conformal maps onto polygons can also approximate maps onto other domains, as long as each boundary point has a neighborhood which is an image of a half-disk by an explicit conformal map. The algorithm is just a way of computing a global conformal map from knowing the local maps around each boundary point; we deal with polygons since the local maps are trivial. The work needed in general is $O(N)$, where $N$ is the number of simple disks needed to cover the boundary (a simple disk is one so that $2 D \cap \Omega$ can be explicitly mapped to a half-disk, with the boundary going to the line segment). In the case of polygons, we can reduce $O(N)$ to $O(n)$ by using arches, but this requires "conformally straightening" two boundary arcs simultaneously. For polygons, we do this with 3-parameter Schwarz-Christoffel maps, but it may not be easy to do for curved boundary segments. For example, local boundary maps are also easy to find for circular arc polygons, but I don't know how to "straighten" pairs of circular arcs (unless they happen to lie on intersecting circles). Thus the method of this paper will compute an $(1+\epsilon)$-quasiconformal map onto a circular arc $n$-gon in time $O(N|\log \epsilon \log \log \epsilon|)$, but I don't yet see how to reduce $N$ to $n$. The special case of finitely bent domains (unions of disks) will be discussed later in detail, and conformal maps onto such domains will be computed as part of the proof of Theorems 1 and 2.

This paper is part of a series of papers that have studied hyperbolic geometry and its relation to conformal mappings [11, 13, 14, 16, 17]. Along the way, many people have contributed helpful comments, advice and encouragement including Raphy Coifman, Tobin Driscoll, David Epstein, John Garnett, Peter Jones, Al Marden, Vlad Markovic, Joe Mitchell, Nick Trefethen, Jack Snoeyink and Steve Vavasis. Many thanks to them and the others who helped me reach the results described here.

Also special thanks to the referees who made a tremendous effort reading and evaluating the manuscript. Their thoughtful and extensive remarks touched on everything from typos to the overall strategy of the proof, and prompted a rewriting which simplified parts of the proof and improved the exposition. The longer the paper, the more important (and more difficult) good writing becomes, and I very much appreciate their help in making this a better paper.

\section{Summary of the Proof}

Now we will summarize our method for computing conformal maps. I hope that even without the precise definitions, this sketch will help motivate what follows and give a "map" for reading the rest of the paper.

Suppose $\Omega$ is a simply connected domain with a polygonal boundary with $n$ sides. Let $\epsilon_{0}$ be the radius of convergence of our Newton-type iteration for representations 
(see Lemma 31). Compute the medial axis of $\Omega$ and use it to break $\Omega$ into $O(n)$ thick and thin pieces (see Sect. 12). Fix a thick piece $\Omega^{\text {thick }}$ and approximate it by a finitely bent region $\Omega^{\mathrm{fb}}$ using Lemma 26 with a "flattening map" that is $(1+\delta)$ quasiconformal. Compute the corresponding bending lamination (Sect. 8), normal crescent decomposition (Sect. 5) and the chain of finitely bent angle scaling domains $\Omega_{0}=\mathbb{D}, \ldots, \Omega_{N}=\Omega^{\mathrm{fb}}$. We will prove that if $\delta$ is small enough and $N$ is large enough (depending only on $\epsilon_{0}$ ), then:

(1) (Starting point) We can construct an $\epsilon_{0} / 2$ representation of $\Omega_{0}=\mathbb{D}$ (trivial).

(2) (Composition step) Given an $\epsilon_{0} / 2$ representation of $\Omega_{k}$ we can construct an $\epsilon_{0}$ representation of $\Omega_{k+1}$ (Lemma 29).

(3) (Improvement step) Given an $\epsilon_{0}$ representation of $\Omega_{k}$ we can compute an $\epsilon_{0} / 2$ representation of $\Omega_{k}$ (Lemma 31).

(4) (Final conversion) Given an $\epsilon_{0} / 2$-representation of $\Omega_{N}=\Omega^{\mathrm{fb}}$ we can construct a $\epsilon_{0}$ representation of $\Omega$ (Lemma 30).

(5) (Iterate to desired accuracy) Given any $\epsilon<\epsilon_{0}$ and a $\epsilon_{0}$-representation of $\Omega$, we can compute an $\epsilon$-representation of $\Omega$ (Lemma 31).

It is important to note that in steps (1)-(4) we only compute maps to a fixed accuracy; just enough to use it as a good starting point for the map onto the next element. Thus the precise timing of these steps is unimportant, as long as it is linear in $n$ with a constant depending only on $\epsilon_{0}, \delta, N$. All these constants will be chosen independent of $n$ and the geometry of $\Omega$, so the total work to get an $\epsilon_{0}$-representation of $\Omega$ is $O(n)$ with an constant independent of $n$ and $\Omega$.

At the final step we use Lemma 31 to iterate until we reach the desired $\epsilon$. By Lemma 31, the $k$ th iteration gives accuracy $\epsilon_{0}^{2^{k}}$ and takes time $O\left(n 2^{k} k\right)$ to perform (with constant depending only on the fixed number $\left.\epsilon_{0}\right)$. Thus $O\left(\log \frac{1}{\epsilon}\right)$ iterations are needed to reach accuracy $\epsilon$. Since the time per iteration grows exponentially at each step, the total time is dominated by the final step, which is $O\left(n \log \frac{1}{\epsilon} \log \log \frac{1}{\epsilon}\right)$.

In an earlier version of this paper, the chain of domains consisted of polygons inscribed in the angle scaling family of finitely bent domains. This was awkward, but avoided some complications of extending the idea of $\epsilon$-representations from polygons to finitely bent domains. This version deals with these complications, in return for a cleaner presentation of the angle scaling chain and inductive steps.

The paper divides roughly into five parts: (1) an expository introduction to the medial axis, $\iota$-map and angle scaling, (2) the construction of the bending lamination, the associated decomposition of $\mathbb{H}$ and our representation of conformal maps, (3) the thick/thin decomposition of polygons and the special properties of thin polygons, (4) constructing the chain of domains connecting $\mathbb{D}$ to $\Omega$ and implementing the composition step on representations, and (5) our iteration for improving representations based on finding approximate solutions of the Beltrami equation by the multipole method. More precisely, the remaining sections are:

Sect. 3 We introduce the medial axis and the hyperbolic dome.

Sect. 4 We discuss Thurston's observation that the dome of a simply connected domain $\Omega$ is isometric to the hyperbolic disk. We show how this gives a mapping $\iota$ from $\partial \Omega$ to $\mathbb{T}$. 
Sect. 5 We introduce the gap/crescent decomposition of a finitely bent domain, the corresponding bending lamination on the disk and construct the angle scaling chain of domains that connects the disk to $\Omega$.

Sect. 6 We show that elements of the angle scaling chain are close in a uniform quasiconformal sense. This is one of the key ideas that makes the whole method work with uniform estimates.

Sect. 7 We prove a technical result used in Sect. 6. We introduce the idea of piecewise Möbius maps and $\epsilon$-Delaunay triangulations to prove that a map which is close to Möbius transformations locally has a global approximation by a hyperbolic biLipschitz function.

Sect. 8 We show the bending lamination of a finitely bent domain can be computed in linear time.

Sect. 9 We cover the bending lamination by $O(n)$ "simple" regions.

Sect. 10 We refine this covering and extend it to a decomposition of $\mathbb{H}$.

Sect. 11 We define an $\epsilon$-representation of a polygonal domain and show such a representation corresponds to a $(1+O(\epsilon))$-quasiconformal map onto the domain.

Sect. 12 We define thick and thin polygons and show that any polygon with $n$ sides can be decomposed into thick and thin pieces with a total of $O(n)$ sides, and with certain estimates on the overlaps of the pieces. We also record some approximation results for conformal maps onto thin polygons.

Sect. 13 We show how to approximate thick polygons by finitely bent domains and define $\epsilon$-representations of conformal maps onto such domains. We use the approximation to define an angle scaling family.

Sect. 14 We show that if a polygon satisfies a strong form of thickness, its finitely bent approximation satisfies a weak form. We use this to show how a representation of one element of the angle scaling family can be used to construct a representation of the next element.

Sect. 15 Assuming we can approximately solve a certain Beltrami equation we show how to update a $\epsilon$-representation to a $\epsilon^{2}$-representation.

Sect. 16 We reduce solving the Beltrami problem to solving a $\bar{\partial}$ problem.

Sect. 17 We show how to quickly solve the $\bar{\partial}$-problem by computing the Beurling transform of a function using the fast multipole method.

Appendix A Background on conformal maps, hyperbolic geometry and quasiconformal mappings. Non-analysts may wish to review some of this material before reading the rest of the paper.

Appendix B We review known results about power series and show that $O(p \log p)$ suffices for all the manipulations needed by the algorithm.

\section{Domes and the Medial Axis: an Introduction}

Here we introduce two closely related geometric objects associated to any planar domain $\Omega$ : the dome of $\Omega$ and the medial axis of $\Omega$. We start with the dome.

Given a closed set $E$ in the plane, we let $C(E)$ denote the convex hull of $E$ in the hyperbolic upper half-space, $\mathbb{H}^{3}=\mathbb{R}_{+}^{3}$. This is the convex hull in $\mathbb{H}^{3}$ of all the infinite hyperbolic geodesics that have both endpoints in $E$ (recall these are exactly 


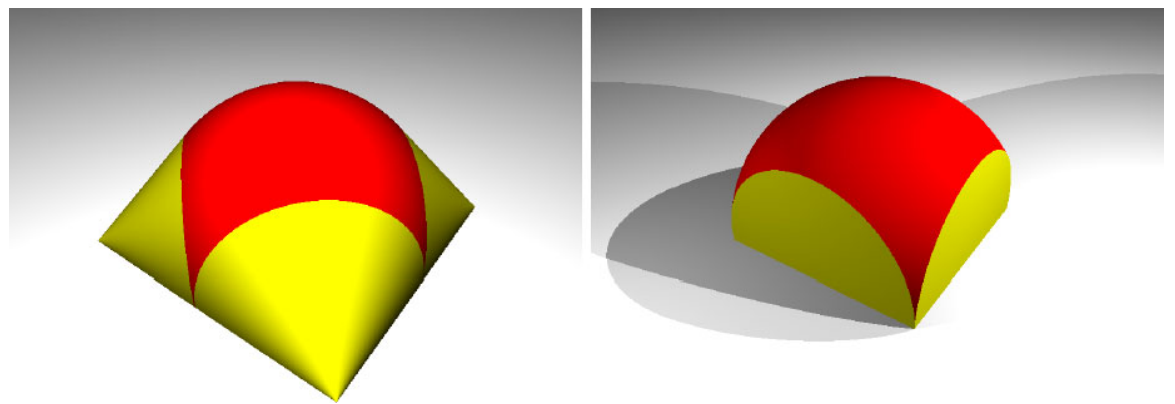

Fig. 8 The lower and upper boundaries of the hyperbolic convex hull of the boundary of a square (left and right figures, respectively). The lower boundary consists of one geodesic face (dark) and four Euclidean cones (lighter). The upper boundary has five geodesic faces (one hemisphere and four vertical). The outside of the square is a finitely bent domain, but the inside is not

the circular arcs in $\mathbb{H}^{3}$ that are orthogonal to $\mathbb{R}^{2}=\partial \mathbb{H}^{3}$ ). One really needs to take the convex hull of the geodesics ending in $E$ and not just the union of these geodesics; for example, if $E$ consists of three points, then there are three such geodesics and these form the "boundary" of an ideal triangle whose interior is also in the convex hull of $E$.

The complement of $C(E)$ is a union of hyperbolic half-spaces. There is one component of $\mathbb{H}^{3} \backslash C(E)$ for each complementary component $\Omega$ of $E$ and this component is the union of hemispheres whose bases are disks in $\Omega$ (also include half-planes and disk complements if $\Omega$ is unbounded). For example, when $E$ is the boundary of a square, the lower and upper boundaries of $C(E)$ are illustrated in Fig. 8.

In this paper, we will focus exclusively on the case of a single, bounded, simply connected domain $\Omega$. In this case, the dome of $\Omega$ is the unique boundary component of the convex set $C\left(\Omega^{c}\right)$. The dome is fairly easy to draw because of the description of $W$ as a union of Euclidean hemispheres with bases in $\Omega$. Moreover,

Lemma 3 Suppose $S_{\Omega}$ is the dome of a simply connected, proper plane domain $\Omega$. Then for every $x \in S_{\Omega}$ there is an open hyperbolic half-space $H$ disjoint from $S_{\Omega}$ so that $x \in \partial H \cap S_{\Omega}$. For any such half-space, $\partial H \cap S_{\Omega}$ contains an infinite geodesic, and its base disk (or half-plane) has boundary that hits $\partial \Omega$ in at least two points.

Proof Let $W=C\left(\Omega^{c}\right)$ be the hyperbolic convex hull of $\Omega^{c}$, so $S_{\Omega}=\partial W$. By definition, $W$ is the intersection of all closed half-spaces that contain it, and from this it is easy to see that any boundary point on $W$ is on the boundary of some closed halfspace that contains $W$. Thus $x$ is also on the boundary of the complementary open half-space $H$ (which must be disjoint from $W$ ). The base of $H$ on $\mathbb{R}^{2}$ is a half-plane or a disk and by conjugating by a Möbius transformation, if necessary, we assume it is the unit disk $D=\mathbb{D}$ and that $H$ contains the point $z=(0,0,1) \in S_{D}$. Clearly, $\partial D$ hits $\partial \Omega$ in at least one point, for otherwise its closure would be contained in another open disk in $\Omega$, whose dome would be strictly higher than $S_{D}$, contradicting that $z \in S_{D} \cap S_{\Omega}$. In fact, $\partial D$ must hit $\partial \Omega$ in at least two points. For suppose it only hit at one point, say $(1,0) \in \mathbb{R}^{2}$. Then for $\epsilon>0$ small enough the disk $D(-2 \epsilon, 1+\epsilon)$ 

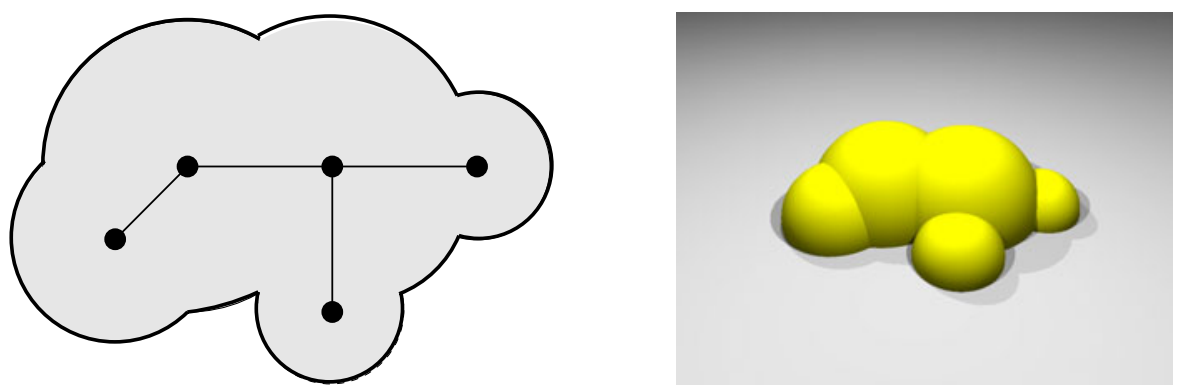

Fig. 9 A finitely bent domain, its medial axis and its dome

would also be in $\Omega$ and its dome would strictly separate $z$ from $S_{\Omega}$. Thus $\partial D$ hits $\partial \Omega$ in at least two points and the geodesic in $\mathbb{R}_{+}^{3}$ between these points lies on the $\partial H \cap S_{D}$, as desired.

Thus each point on the dome is also on the dome of a disk in $\Omega$ whose boundary hits $\partial \Omega$ in at least two points. Such a disk is called a "medial axis disk" for $\Omega$ and the set of centers of such disks is called the medial axis of $\Omega$, denoted $\operatorname{MA}(\Omega)$. (The centers, together with the radii, is usually called the medial axis transform of $\Omega$, $\operatorname{MAT}(\Omega)$.) It is easy to see that the $\Omega$ is the union of its medial axis disks, and so it determined by $\operatorname{MAT}(\Omega)$.

The dome is easiest to visualize when $\Omega$ is a finite union of disks, e.g., see Fig. 9. Such a domain will be called "finitely bent" because the dome consists of a finite union of geodesic faces (each contained on a geodesic plane in $\mathbb{H}^{3}$, i.e., a Euclidean hemisphere or vertical plane) which are joined along infinite geodesics called the bending geodesics.

When we are given a finitely bent domain $\Omega$ we shall always assume we are given a complete list of disks in $\Omega$ whose boundaries hit $\partial \Omega$ in at least three points. Then every face of the dome corresponds to a hemisphere that has one of these disks as its base. This is slightly different than just giving a list of disks whose union is $\Omega$; in Fig. 10 we show a domain that is a union of four disks $\Omega=D(1,1) \cup D(i, 1) \cup$ $D(-1,1) \cup D(-i, 1)$ but that contains a fifth disk, $D(0, \sqrt{2})$, which also corresponds to a face on the dome of $\Omega$.

The faces of the dome of a finitely bent domain form the vertices of a finite tree, with adjacency defined by having an infinite geodesic edge in common. This induces a tree structure on the maximal disks in the base domain: disks that hit exactly two boundary points are interior points of edges of the tree and disks that hit three or more points are the vertices.

Lemma 4 For any tree the number of vertices of degree three or greater is less than the number of degree one vertices.

The proof is easy and left to the reader (remove a degree one vertex and use induction). So if $\Omega$ can be written as a union of $n$ disks in any way, there are at most $2 n$ vertices of the medial axis. 

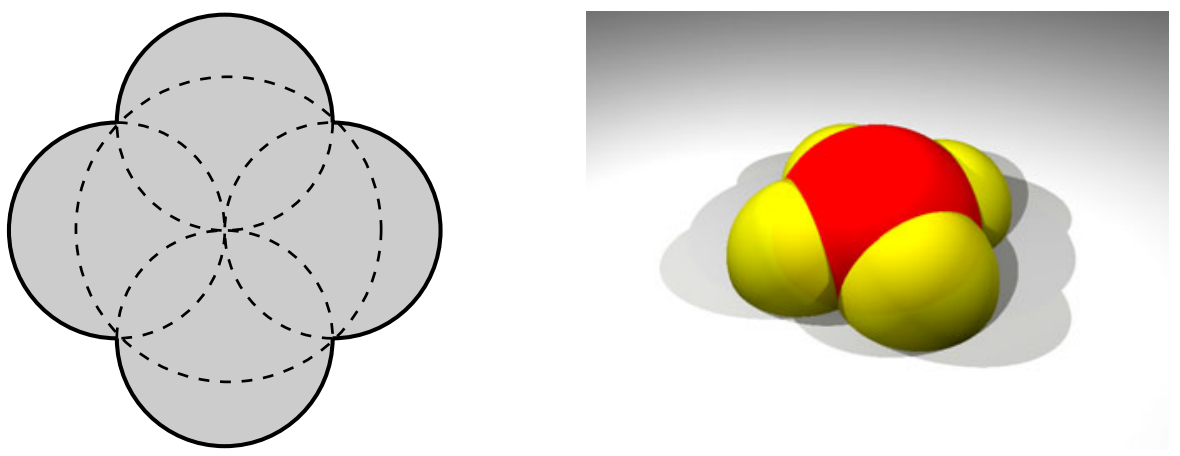

Fig. 10 A domain that is a union of four disks, but which has five faces on the dome because of a "hidden" maximal disk

For polygons the medial axis is also a finite tree, but now there are three types of edges: (1) edge-edge bisectors that are straight line segments equidistant from two edges, (2) point-point bisectors, which are straight line segments equidistant from two vertices, or (3) point-edge bisectors, which are parabolic arcs equidistant from a vertex and an edge. For an $n$-gon the medial axis has at most $O(n)$ vertices (it is not hard to show $2 n+3$ works).

To illustrate these ideas we show a few polygons, along with their medial axes and their domes. The dome of a polygon is naturally divided into kinds of pieces: (1) a hyperbolic geodesic face corresponding to a vertex of the medial axis of degree three or more, (2) a cylinder or cone corresponding to sweeping a hemisphere along a bisector of two edges, or (3) sweeping a hemisphere along the parabolic arc of a point-edge bisector. Disks corresponding to the interiors of point-point bisector edges do not contribute to the dome since the union of the two disks at the endpoints of this edge contain all the disks corresponding to the interior points.

In the dome of a convex polygon, only the first two types of pieces can occur. These are illustrated in Fig. 11. The third type of medial axis arc can occur in nonconvex domains, as illustrated in the polygonal "corner" in Fig. 12.

The medial axis also suggests a way of approximating any domain by a finite union of disks; simply take a finite subset of the medial axis so that the corresponding union of medial axis disks is connected. The medial axis of such a union consists of one vertex for each geodesic face in the dome and straight lines connecting the vertices corresponding to adjacent faces. A polygon, its medial axis and a finitely bent approximation are shown in Fig. 13. In Fig. 14, we show the domes of the polygon and its approximation. The process of approximating a polygon by a finitely bent region will be discussed in greater detail in Sect. 13. Alternate approximations of polygons by disks coming from circumcircles of a triangulation of the polygon are used in [16, 49].

The medial axis is a fundamental concept of geometry that seems to have been rediscovered many times and goes by several names: medial axis, skeleton, symmetry set, cut locus (defined as the closure of the medial axis in [134]), equidistant set, ridge set (think of an island where the elevation is proportional to the distance to the sea), wildfire set (think of a fire started simultaneously along the boundary that burns 

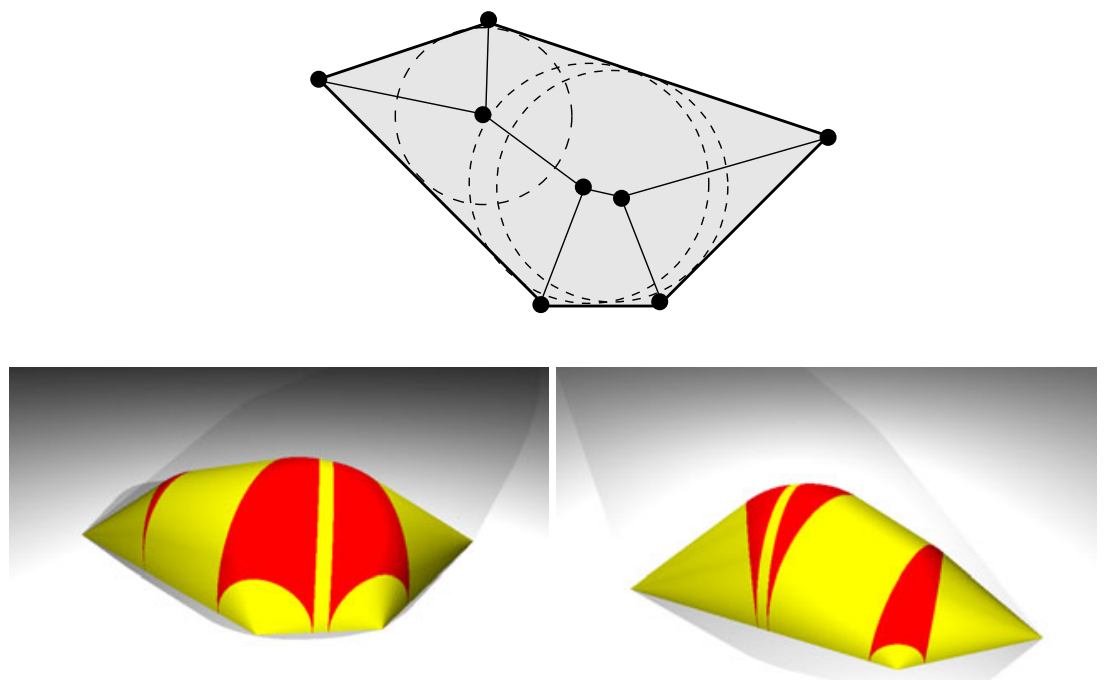

Fig. 11 The medial axis and dome of a convex region. This dome has three geodesic faces that are shaded darker (these correspond to vertices of the medial axis); the lighter parts of the dome are Euclidean cones that correspond to edges of the medial axis. The dome is shown from two different directions
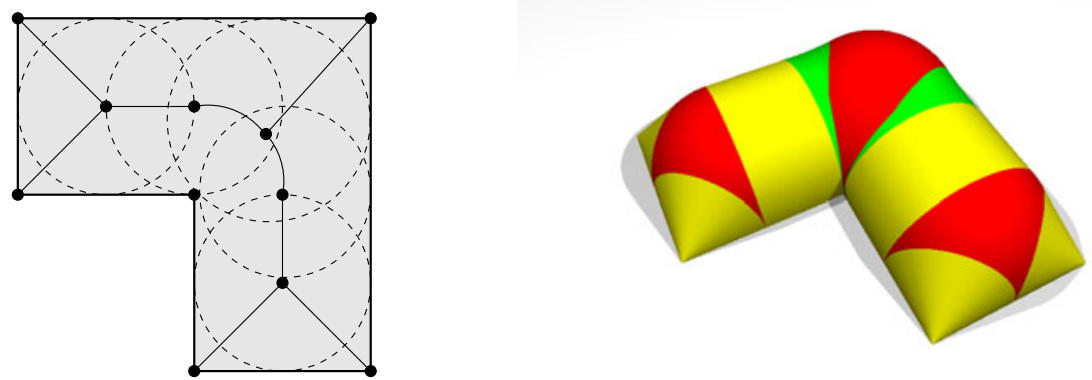

Fig. 12 The dome of a "corner". The darkest shading are geodesic faces (vertices of the medial axis); the lightest are Euclidean cones or cylinders (edge-edge bisectors in the medial axis). The medium shading illustrates the third type of medial axis edge that can occur: the parabolic bisector of a point and a line

inward at a constant rate). The earliest reference I am aware of is a 1945 paper of Erdős [57], where he proves the medial axis (he calls it " $M_{2}$ ") of a planar domain has Hausdorff dimension 1.

In some parts of the literature, the medial axis is confused with the set of centers of maximal disks in $\Omega$, that, following [62], we will call the central set of $\Omega$. For polygons the two sets are the same, but in general they are not (e.g., the parabolic 

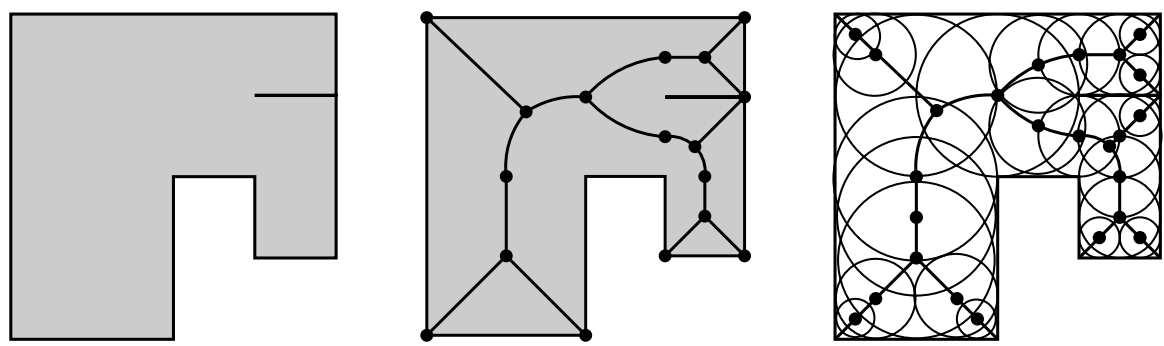

Fig. 13 A (non-simple) polygon, its medial axis and a finitely bent approximation
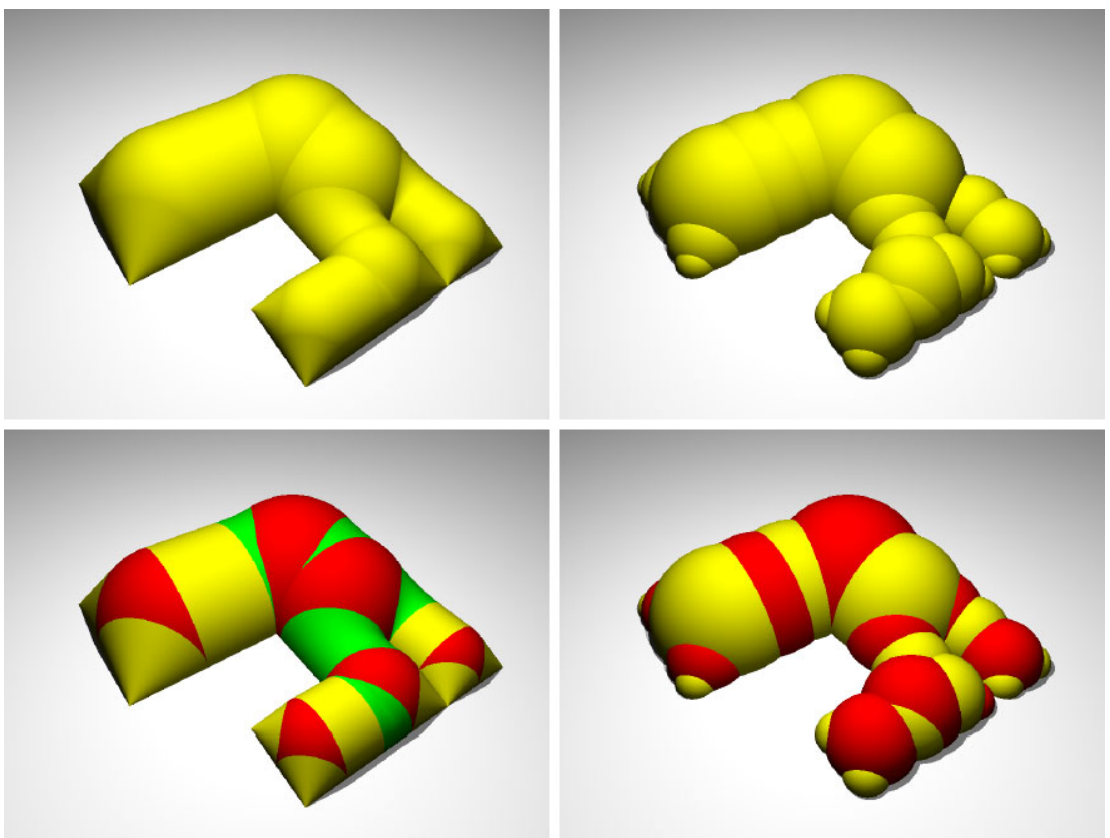

Fig. 14 On the top left is the dome of the polygon $P_{2}$ and on the top right is the dome of the finitely bent approximation $\Omega_{2}$. Below each, we have redrawn the domes, but with different sections shaded differently. For $P_{2}$, regions corresponding to different edges of the medial axis colored differently. For $\Omega_{2}$, the dome is a union of geodesic faces (which form the vertices of a tree) and adjacent faces are shaded in alternating dark and light

region $\Omega=\left\{(x, y): y>x^{2}\right\}$ contains a maximal disk that is only tangent at the origin). More dramatically, the medial axis of a planar domain always has $\sigma$-finite 1-dimensional measure [62], but the central set can have Hausdorff dimension 2 [15]. Some papers in the mathematical literature that deal with the medial axis include $[6,25,50,59,68,69,80,94,95,123]$.

In the computer science literature, the medial axis is credited to Blum who introduced it to describe biological shapes [19-21]. A few papers consider the theory of the medial axis (e.g., [34-37, 114, 134]), but most deal with algorithms for computing it and with applications to areas like pattern recognition, robotic motion, control of 
Fig. 15 This shows the Voronoi cells when all the edges and vertices are sites. However, the dashed edges must be removed to give the medial axis

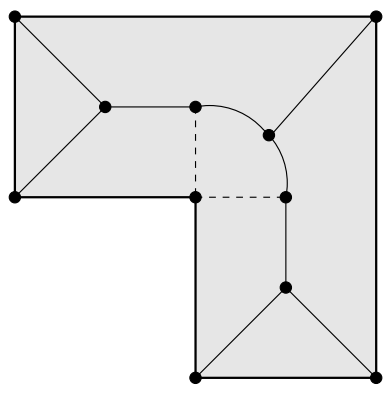

cutting tools, sphere packing and mesh generation. A sample of such papers includes: [28, 29, 32, 41, 58, 65, 71, 77, 78, 84, 88-92, 102-104, 112, 113, 131, 135, 137].

Given a finite collection of disjoint sets (called sites), the corresponding Voronoi diagram divides the plane according to which site a point is closest to. The medial axis of a polygon $P$ is a Voronoi diagram for the interior of $P$ where the sites are the complementary arcs in $P$ of the convex vertices (i.e., interior angle $<\pi$ ) and distance is measured within $P$. Equivalently, one can compute the medial axis by taking the Voronoi diagram for the polygon with all edges and vertices as sites and then removing the cell boundaries that terminate at a concave vertex (one with angle $\leq \pi)$; see Fig. 15. Thus the medial axis can be computed by using algorithms for computing generalized Voronoi diagrams. Voronoi diagrams were defined by Voronoi in [130], but go back at least to Dirichlet [47] (indeed, in the theory of Kleinian groups the Voronoi cells of an orbit are called Dirichlet fundamental domains). For more about Voronoi diagrams, see, e.g., [4, 5, 8, 60, 61, 100, 105].

It is a theorem of Chin, Snoeyink and Wang that the medial axis of a simple $n$-gon can be computed in $O(n)$ time. I am not aware that their $O(n)$ algorithm has been implemented, since it depends on the intricate algorithm of Chazelle that triangulates polygons in linear time. However, other asymptotically slower methods (e.g., $O(n \log n))$ have been implemented, and in practice the computation of the medial axis in $\mathbb{R}^{2}$ is not considered a "bottleneck"; see [136, 137].

The basic strategy of the linear time algorithm of Chin, Snoeyink and Wang is fairly simple (although the details are not): (1) decompose the polygon into simpler polygonal pieces called monotone histograms using at most $O(n)$ new edges, (2) compute the Voronoi diagram for each piece with work $O(k)$ for a piece with $k$ sides, and finally (3) merge the Voronoi diagrams of the pieces using at most $O(n)$ work.

The first step is accomplished using a celebrated result of Chazelle [30] that one can cut interior of $P$ into trapezoids with vertical sides in linear time (this is equivalent to triangulating the polygon in linear time). Klein and Lingas [86] showed how to use Chazelle's result to cut a polygon into "pseudo-normal histograms"; Chin, Snoeyink and Wang then show how to cut these into monotone histograms.

The next step is to show that the Voronoi diagram of a monotone histogram can be computed in linear time. The argument given in [33] follows the elegant argument of Aggarwal, Guibas, Saxe and Shor for the case of convex domains. In [1], the four authors use duality to reduce the problem to finding the three dimensional convex 
hull of $n$ points whose vertical projections onto the plane are the vertices of a convex polygon.

The final step is to merge the Voronoi diagrams of all the pieces. The merge lemma used in [33] states:

Lemma 5 Let $Q$ be a polygon that is divided into two subpolygons $Q_{1}$ and $Q_{2}$ by a diagonal e (i.e., an line segment in $P$ whose endpoints are vertices of $P$ ). Let $S_{1}$ be a subset sites (vertices and edges) in $Q_{1}$ and $S_{2}$ a subset of sites in $Q_{2}$. Given the Voronoi diagrams for $S_{1}$ in $Q_{1}$ and for $S_{2}$ in $Q_{2}$, one can obtain the Voronoi diagram for $S=S_{1} \cup S_{2}$ in $Q$ in time proportional to number of Voronoi edges for $S_{1}$ and $S_{2}$ that intersect $e$ and the number of new edges that are added.

This type of result was first used by Shamos and Hoey [111] and has been adapted by many authors since.

\section{The Dome is the Disk}

The two main results about the dome of $\Omega$ say that (1) it is isometric to the hyperbolic disk and (2) it is "almost isometric" to the base domain $\Omega$. More precisely, equip the dome with the hyperbolic path metric $\rho_{S}$ (shortest hyperbolic length of a path connecting two points and staying on the surface).

Theorem 6 (Thurston [124]) Suppose $\Omega$ is a simply connected plane domain (other than the whole plane or the complement of a circular arc) and let $S$ be its dome. Then $\left(S, \rho_{S}\right)$ is isometric to the hyperbolic unit disk. We will denote the isometry by $\iota: S \rightarrow \mathbb{D}$.

Theorem 7 (Sullivan [121], Epstein-Marden [52]) Suppose $\Omega$ is a simply connected plane domain (other than the whole plane or the complement of a circular arc). There is a $K$-quasiconformal map $\sigma: \Omega \rightarrow S$ that extends continuously to the identity on the boundary ( $K$ is independent of $\Omega$ ).

In fact, there is a biLipschitz map between $\Omega$ and its dome (each with their hyperbolic metric; see Theorem 49), but we will only use the quasiconformal version of the result. We place the additional restriction that $\Omega$ is not the complement of a circular arc because in that case the convex hull of $\partial \Omega$ is a hyperbolic half-plane and the dome should be interpreted as two copies of this half-plane joined along its edge with bending angle $\pi$. In order to simplify the discussion here, we simply omit this case (with the correct interpretations the results above still hold in this case; this is discussed in complete detail in Sect. 5 of [54]).

Explicit estimates of the constant in the Sullivan-Epstein-Marden theorem are given elsewhere in the literature. For example, it is proven in [14] that one can take $K=7.82$. The estimates $K \approx 80$ and $K \leq 13.88$ are given in [52] and [54], respectively.

Although we will not use it here, it is worth noting that both these theorems have their origin in the theory hyperbolic of 3-manifolds. Such a manifold $M$ is a quotient 
of the hyperbolic half-space, $\mathbb{H}^{3}$, by a discrete group $G$ of isometries. The orbit of any point under this group accumulates only on the boundary of the half-space and the accumulation set (which is independent of the orbit except in trivial cases) is called the limit set $\Lambda$. The complement $\Omega$ of $\Lambda$ in the boundary of hyperbolic space is called the ordinary set. The group $G$ acts discontinuously on $\Omega$ and $\partial_{\infty} M=\Omega / G$ is called the "boundary at infinity" of $M$. This is a Riemann surface (possibly with branch points). The manifold $M$ contains closed geodesics and the closed convex hull of these is called the convex core of $M$ and denoted $C(M)$. The lift of the convex core to $\mathbb{H}^{3}$ is the hyperbolic convex hull of the limit set and its boundary is the dome of the ordinary set. Thus $\partial C(M)$ is just the quotient of this dome by the group $G$. Theorem 6 implies that the boundary of $C(M)$ is a surface of constant negative curvature, i.e., is isomorphic to the hyperbolic disk modulo a group of isometries. Theorem 7 says that $\partial_{\infty} M$ and $\partial C(M)$ are homeomorphic, indeed, are quasiconformal images of each other with respect to their hyperbolic metrics. This fact was needed in the proof of Thurston's hyperbolization theorem for 3-manifolds that fiber over the circle.

The proof of Theorem 6 for finitely bent domains simply consists of observing that if we deform the dome by bending it along a bending geodesic, we don't change the path metric at all. Moreover, a finite number of such deformations converts a finitely bent dome into a hemisphere, and this is obviously isomorphic to the hyperbolic disk. More precisely, we are using the following simple lemma.

Lemma 8 Suppose two surfaces $S_{1}, S_{2}$ in $\mathbb{H}^{3}$ are joined along a infinite hyperbolic geodesic and suppose $\sigma$ is an elliptic Möbius transformation of $\mathbb{H}^{3}$ that fixes this geodesic. Then a map to another surface that equals the identity on $S_{1}$ and equals $\sigma$ on $S_{2}$ is an isometry between the path metric on $S_{1} \cup S_{2}$ and the path metric on the image.

Proof This becomes obvious is one normalizes so that the geodesic in question becomes a vertical line and $\sigma$ becomes a (Euclidean) rotation around it, since it is then clear that the length of any path is left unchanged.

Theorem 6 then follows by taking a finitely bent surface and "unbending" it one geodesic at a time, i.e., we can map it to a hemisphere by a series of maps, each of which is an isometry by the lemma. Since a hemisphere is isometric to the disk, we are done. In Fig. 16, we illustrate the bending along a geodesic for a dome with two faces.

This proof gives us a geometric interpretation of the map $\iota: \partial \Omega \rightarrow \partial \mathbb{D}$. The disks making up a finitely bent domain have a tree structure and if $\Omega$ is finitely bent then we fix a root disk $D_{0}$ and write $\Omega=D_{0} \cup \bigcup_{j} D_{j} \backslash D_{j}^{*}$, where $D_{j}^{*}$ denotes the parent disk of $D_{j}$. This gives $\Omega \backslash D_{0}$ as a union of crescents; see Fig. 17. We call these "tangential" crescents since one edge of the crescent follows $\partial \Omega$ near each vertex (and to differentiate them from the "normal" crescents we will introduce later).

Each crescent in the tangential crescent decomposition has an "inner edge" (the one in the boundary of $D_{j}^{*}$ ) and an "outer edge" (the other one) and there is a unique elliptic Möbius transformation that maps the outer edge to the inner one, fixing the two vertices of the crescent (this is just the restriction to the plane of the Möbius 


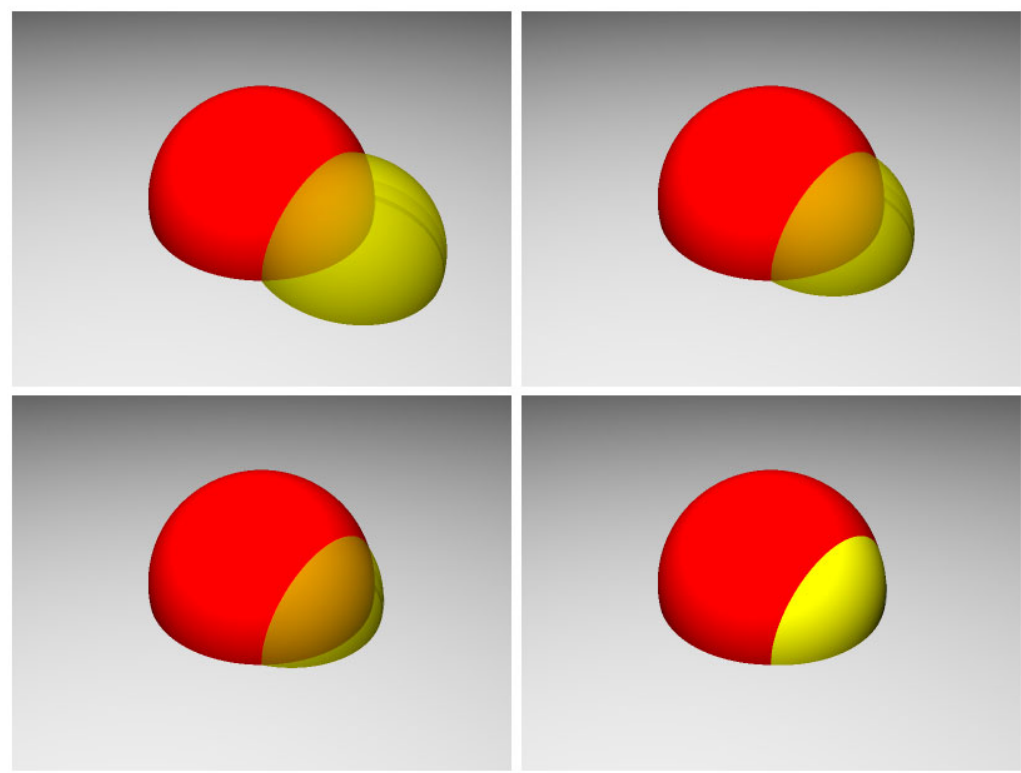

Fig. 16 A dome consisting of two geodesic faces joined along an infinite geodesic. By bending the dome along the geodesic, we get a one-parameter, isometric family of surfaces ending with a hemisphere, which is obviously isometric to the hyperbolic disk

transformation of $\mathbb{H}^{3}$ that removes the bending along the corresponding bending geodesic). The map $\iota: \partial \Omega \rightarrow \partial \mathbb{D}$ is the composition of these maps along a path of crescents that connects an arc on $\partial \Omega$ to an arc on $\partial \mathbb{D}$.

An alternate way to think of this is to foliate each crescent $D_{j} \backslash D_{j}^{*}$ by circular arcs that are orthogonal to both boundary arcs. This gives a foliation of $\Omega \backslash D_{0}$ by piecewise circular curves that connect $x \in \partial \Omega$ to $\iota(x) \in \partial \mathbb{D}$. On the left of Fig. 17, we have sketched the foliation in each of the crescents for a particular finitely bent domain (but without attempting to line up the leaves in different crescents) and on the right we have plotted the trajectories of a couple of boundary points that correspond to the vertices of the polygon we have approximated. This is the description given in the introduction. Some further examples are illustrated in Fig. 18.

Theorem 7 implies that the mapping $\iota: \partial \Omega \rightarrow \partial \mathbb{D}$ has a quasiconformal extension to a map $\Omega \rightarrow \mathbb{D}$ that is $K$-quasiconformal with a bound $K$, independent of $\Omega$. Thus the geometric map we have described above is a rough approximation to the boundary values of the Riemann map. It is surprising (at least to the author) that there is such a simple, geometrically defined map that is close to the Riemann map with estimates independent of the domain.

\section{The Nearest Point Retraction and Normal Crescents}

In the previous section, we defined the map $\iota$ and interpreted it geometrically by collapsing tangential crescents. In this section, we will interpret $\iota$ as collapsing crescents 

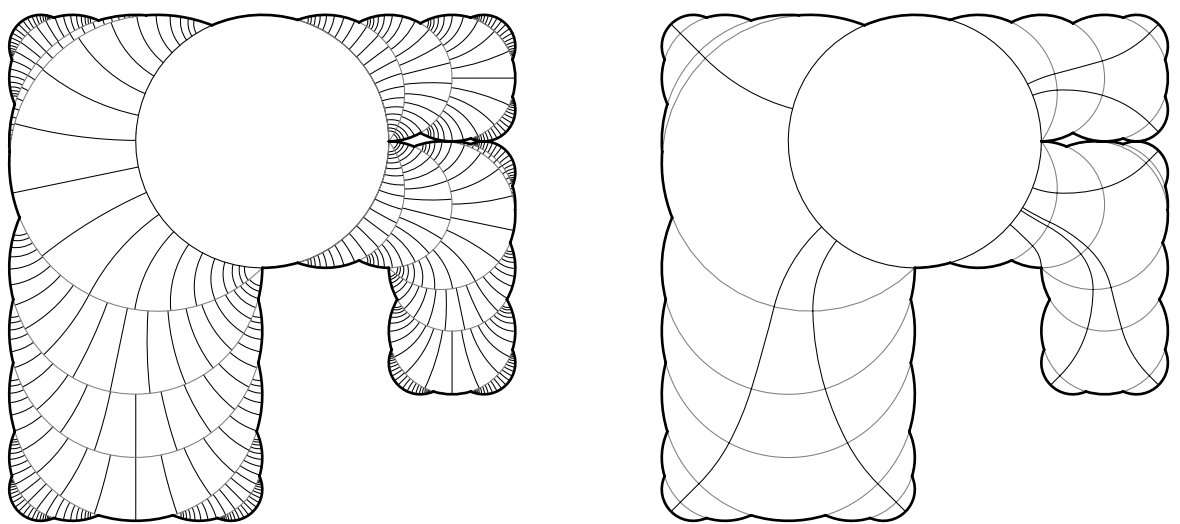

Fig. 17 On the left is the foliation by orthogonal arcs in the tangential crescents. On the right we start at the vertices on the boundary of $\Omega_{2}$ follow the corresponding trajectories of the vertices. Where these trajectories land on the circle are the $\iota$ images of the vertices
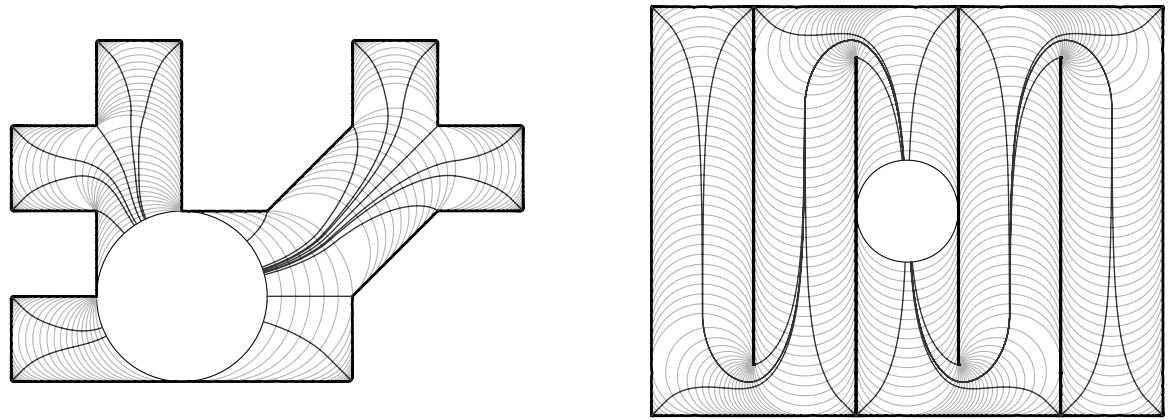

Fig. 18 The medial axis flow for two more polygons which have been approximated by unions of medial axis disks. This flow defines the iota map from $\partial \Omega$ to the chosen root disk of the medial axis

from a different decomposition of $\Omega$ that more closely approximates the geometry of the dome.

Recall that $S$ is the boundary of a convex set in $\mathbb{H}^{3}$, so that the nearest point retraction defines a Lipschitz map of the complement of this set onto its boundary. This map can be extended to $\Omega \subset \partial \mathbb{H}^{3}=\mathbb{R}^{2}$ as follows: given a point $z \in \Omega$, define nearest point retraction $R: \Omega \rightarrow S$ by expanding horoball tangent at $z \in \Omega$ until it first hits $S$ at $R(z)$ (a horoball in $\mathbb{H}^{3}$ is a Euclidean ball tangent to the boundary); see Fig. 19.

Note that the map need not be $1-1$, i.e., two points in $\Omega$ can map to the same point on the dome. Thus it can't always be quasiconformal or even be a homeomorphism. However, it is always a quasi-isometry with bounds independent of $\Omega$ and this implies that there is a quasiconformal map from $\Omega$ to its dome with the same boundary values by Theorem 49. This implies Theorem 7, e.g., see [11]. Moreover, $R$ is quasiconformal in some special cases; e.g., Epstein, Marden and Markovic prove in [55] that for Euclidean convex domains the retraction map is 2-quasiconformal. 
Fig. 19 Defining the retraction map $R: \Omega \rightarrow S$ : expand a sphere tangent at $z$ until it touches $S$ at $R(z)$. This map need not be $1-1$

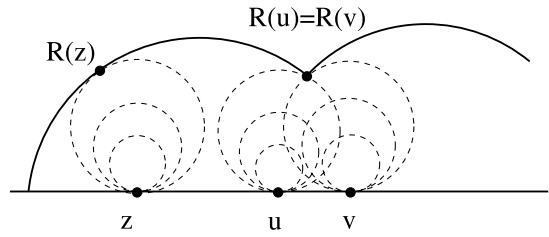

This map is called the nearest point retraction because it is the continuous extension to the boundary of the map in $\mathbb{H}^{3}$ that sends a point to the nearest point of $S$ in the hyperbolic metric, $\rho_{\mathbb{H}^{3}}$; see Appendix A for the definition of the hyperbolic metric on $\mathbb{D}$ and $\mathbb{H}^{3}$. The surface $S$ has an important related metric, $\rho_{S}$. This is the hyperbolic path metric on $S$ defined by taking the shortest hyperbolic length of all paths that connect two points and stay on $S$. Clearly, $\left.\rho_{\mathbb{H}^{3}}\right|_{S} \leq \rho_{S}$. The base domain of $\Omega$ has its own hyperbolic metric, $\rho_{\Omega}$, obtained by transporting the hyperbolic metric on $\mathbb{D}$ by any conformal map.

The nearest retraction map $R$ is $C$-Lipschitz from $\rho_{\Omega}$ to $\rho_{S}$ for some $C<\infty$ (e.g., see [14]). It is easy to prove this for some $C$; the sharp estimate of $C=2$ is given in [53] and earlier results are given in [24, 26, 52].

Now suppose $\Omega$ is a finitely bent domain. Then the dome $S$ of $\Omega$ is a finite union of geodesic faces. On the interior of each face the retraction map has a well defined inverse and the images of the faces under $R^{-1}$ are called the "gaps". The inverse images of the bending geodesics are crescents that separate the gaps. These are called "normal crescents" since their two boundary arcs are perpendicular to the two arcs of $\partial \Omega$ that meet at the common vertex. Therefore, we will call this decomposition of $\Omega$ the "normal crescent decomposition". Refer back to Fig. 6; that picture shows a polygon, a finitely bent approximation, the normal crescent decomposition and the dome; see Fig. 20 for more examples of gap/crescent decompositions.

If a gap $G$ corresponds to a face $F \subset S$ then $G \subset D$, the disk in $\Omega$ that is the base of the hyperplane containing the face $F$. We will call $D$ the "base disk" of $G$ Moreover, $G$ is the hyperbolic convex hull in $D$ of the set where $F$ meets $\partial \Omega$. The angle of a normal crescent $C$ is the same as the angle made by the faces of the dome that meet at the corresponding bending geodesic. $C$ is foliated by circular arcs that are orthogonal to both boundary arcs and each of these arcs is collapsed to single point by $R$. Thus for a finitely bent domain $\Omega, R$ will never be a homeomorphism (unless $\Omega$ is a disk).

The two vertices of each normal crescent are also the vertices of a crescent in the tangential crescent decomposition of $\Omega$. Moreover, corresponding crescents from the two decompositions have the same angle, and hence are simply images of each other by a $\pi / 2$ elliptic rotation around the two common vertices; see Fig. 21. Collapsing the two types of crescents simply gives the two different continuous extensions to the interior of the same map on the boundary (namely $\iota$ ).

Both decompositions cut $\Omega$ into a "disk" and a union of crescents. In the tangential decomposition, it is a single connected disk, but in the normal decomposition the disk itself is broken into pieces called the gaps. The map $\varphi=\iota \circ R: \Omega \rightarrow S \rightarrow \mathbb{D}$ is Möbius on each gap and collapses every crescent to a hyperbolic geodesic in $\mathbb{D}$, thus the disk is written as a union of Möbius images of gaps; for example, see Fig. 22. The 

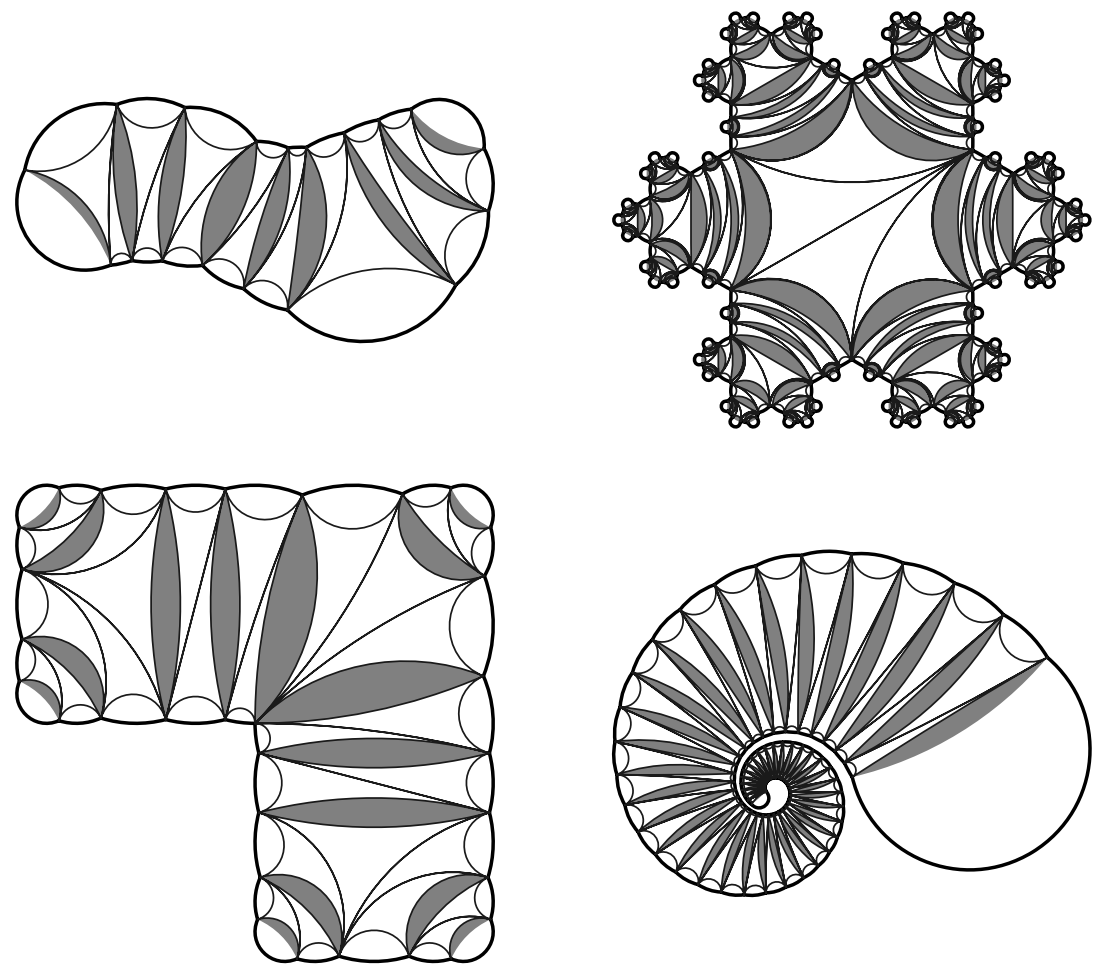

Fig. 20 Normal crescent decompositions for some finitely bent domains. Also drawn are arcs triangulating the gaps. These are added to make the bending lamination complete (see Sect. 8)
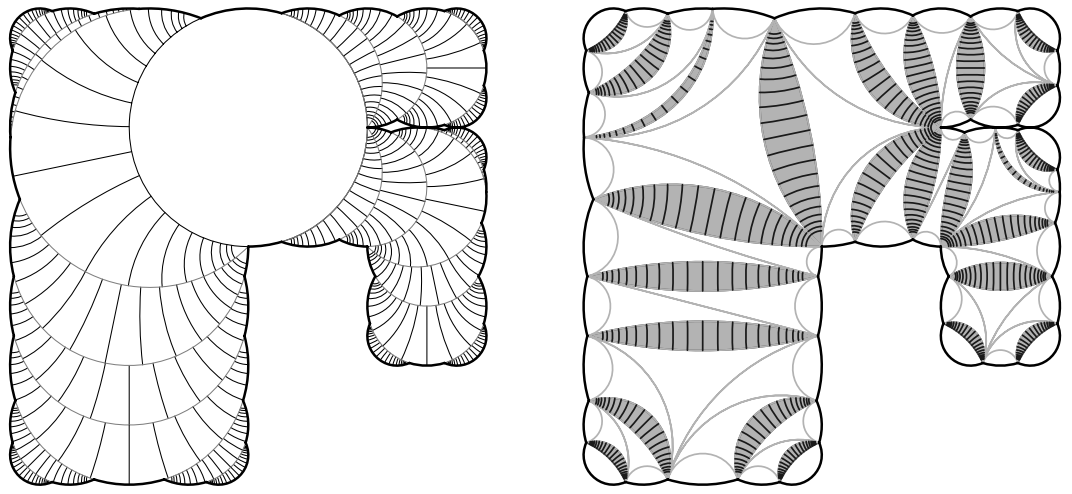

Fig. 21 The tangential and normal crescent decomposition for a domain. There is a 1-1 correspondence between crescents in the two pictures; corresponding crescents have the same vertices and same angle, but are rotated by $\pi / 2$ 

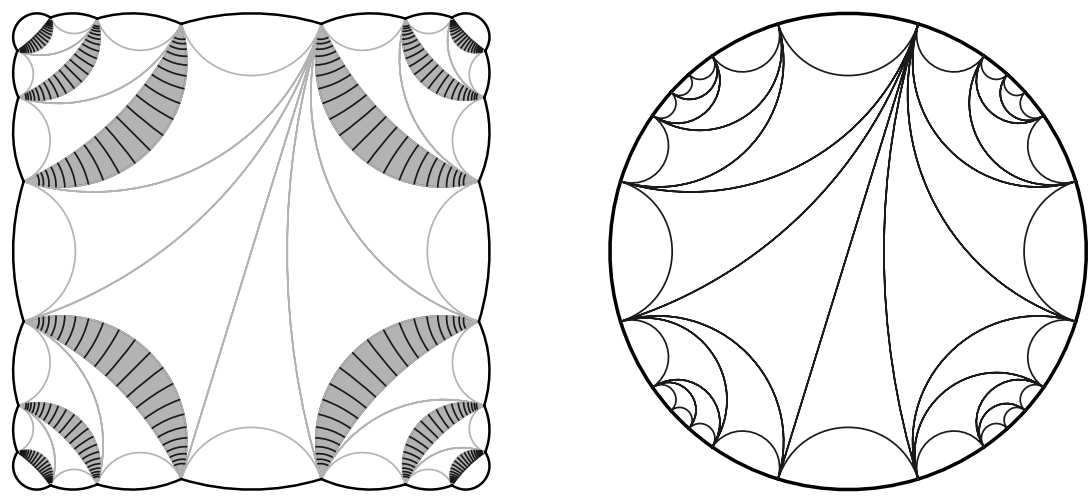

Fig. 22 A normal crescent decomposition of a square and the corresponding bending lamination in the disk. We can recover the decomposition from the lamination by "thickening" each geodesics to a crescent of the correct angle
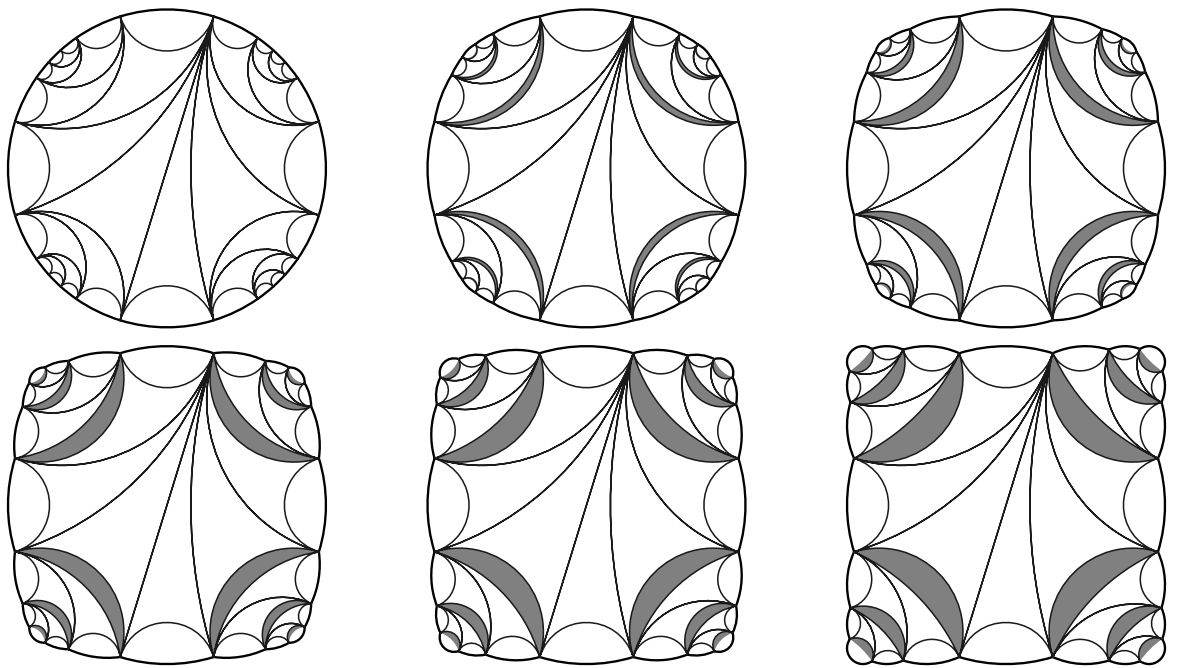

Fig. 23 The one parameter family connecting the disk to a finitely bent approximation of the square. In each picture, the angles have been multiplied by $t=0,0.2,0.4,0.6,0.8,1$

picture on the left shows a normal crescent decomposition of a square and on the right are the $\varphi$ images of the gaps in the disk. The images of the crescents is a finite union of geodesics that is called the "bending lamination" of $\Omega$. If we record the angle of each crescent and assign it to the corresponding geodesic in the bending lamination, then we get a "measured lamination", and this data is enough to recover $\Omega$, up to a Möbius image. We will discuss laminations further in Sect. 8.

We can recover the normal crescent decomposition from the bending lamination by "thickening" each bending geodesic to a crescent of the correct angle, and moving the gaps by the corresponding elliptic transformations. If we do this continuously, we 

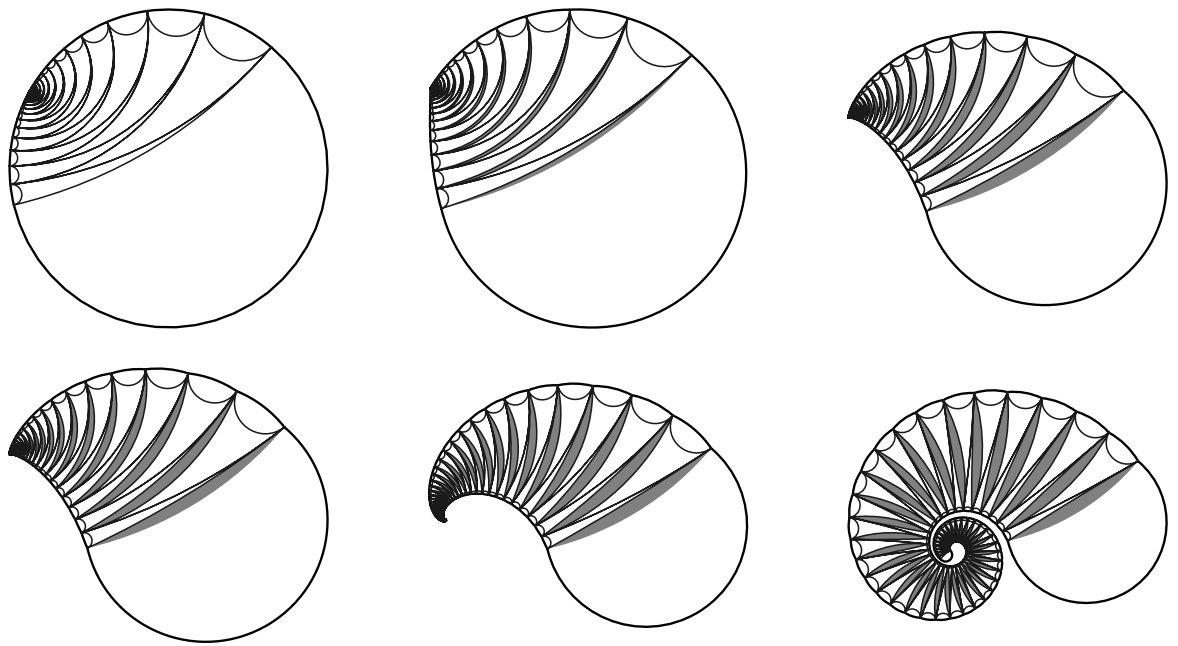

Fig. 24 An approximate logarithmic spiral with $t=0,0.2,0.4,0.6,0.8,1$. Logarithmic spirals were used by Epstein and Markovic in [56] to disprove Thurston's $K=2$ conjecture. They showed that (in a precise sense) certain spirals have too much gray
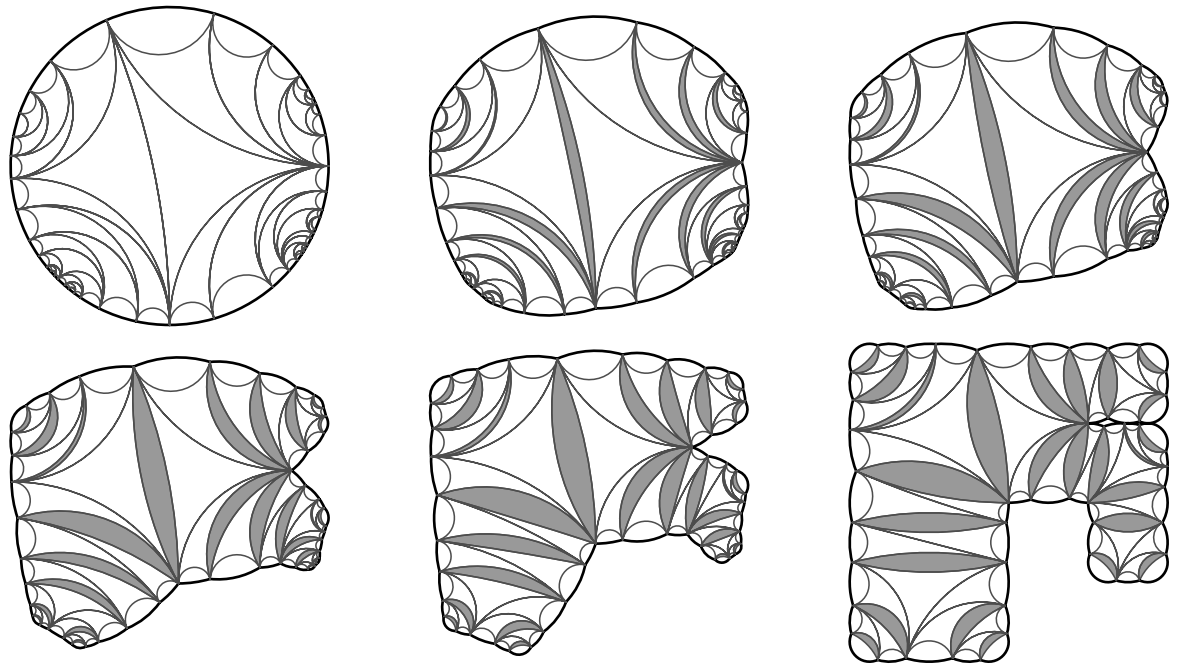

Fig. 25 The domain from Fig. 21 with $t=0,0.2,0.4,0.6,0.8,1$

obtain a family of domains connecting the disk to $\Omega$. For $0 \leq t \leq 1$, let $\Omega_{t}$ be the domain obtained by replacing a crescent of angle $\alpha$ in the normal decomposition by a crescent or angle $t \alpha$; see Figs. 23, 24, 25, 26 for some examples of these 1-parameter families. In general, the intermediate domains need not be planar, but we can think of them as Riemann surfaces that are constructed by gluing together crescents and gaps of given sizes along their edges. Figure 26 shows an example where the intermediate 

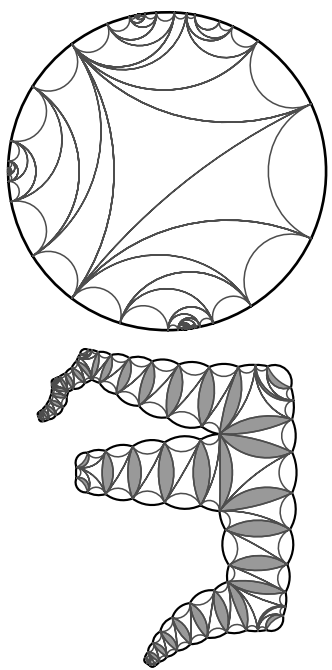
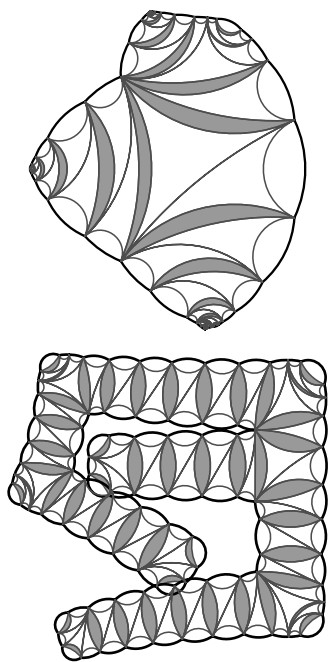
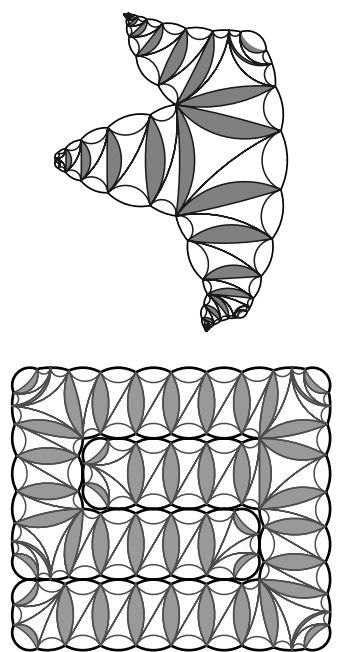

Fig. 26 An example where intermediate domains need not be planar. The pictures correspond to multiplying the angles by $t=0,0.4,0.8,0.95,0.99,1$. Note that the parameter must be very close to 1 before we see the longer corridors clearly

domains are not planar (one sees some small overlap for parameter value $t=0.99$; bigger overlaps could be produced by other examples).

Given a pair of domains $\Omega_{s}, \Omega_{t}$ with $0 \leq s<t \leq 1$, let $\iota_{s, t}: \partial \Omega_{t} \rightarrow \partial \Omega_{s}$ be the obvious boundary map obtained multiplying the angle of each crescent by $s / t$. We will extend this boundary map to the interiors by writing each crescent $C$ in $\Omega_{t}$ of angle $\alpha$ as a union of crescents $C_{1}$, of angle $\alpha s / t$ and $C_{2}$, of angle $\alpha(1-s / t)$. On $C_{1}$ we collapse each leaf of the $E$-foliation to a point (hence $C_{1}$ is maps to a circular arc) and we let our map be Möbius on $C_{2}$. By continuity, this Möbius transformation would have to agree with the map on the gap that is adjacent to $C_{2}$. We will let $\varphi_{s, t}: \Omega_{t} \rightarrow \Omega_{s}$ denote this map. Let $\rho_{s}=\rho_{\Omega_{s}}$ denote the hyperbolic metric on $\Omega_{s}$. Suppose $N$ is a large integer and choose points $t_{0}=0, t_{1}=\frac{1}{N}, \ldots, t_{N}=1$. Let $\Omega_{k}=$ $\Omega_{t_{k}}$ for $k=0, \ldots, N$. Let $\varphi_{k}: \Omega_{k+1} \rightarrow \Omega_{k}$ be defined by $\varphi_{k}=\varphi_{\frac{k}{n}, \frac{k+1}{n}}$.

\section{$6 \varphi_{s, t}$ is a Quasi-isometry}

As noted before, the retraction map $R: \Omega \rightarrow S$ is a quasi-isometry. Thus $\varphi=\iota \circ R$ : $\Omega \rightarrow \mathbb{D}$ is also a quasi-isometry between the hyperbolic metrics. The same is true for the maps $\varphi_{s, t}$ for any $0 \leq s<t \leq 1$, with constant bounded by $O(|s-t|)$. This result is the goal of this section and the next.

Consider the bending lamination $\Gamma$ associated to a finitely bent domain $\Omega$. Suppose a hyperbolic $r$-ball hits geodesics in $\Gamma$ with angles $\alpha_{1}, \ldots, \alpha_{m}$. We want to show that there is an upper bound $\sum_{j} \alpha_{j} \leq B(r)$ that only depends on $r$; see $[14,52]$ for some variations of this idea. Estimates of $B$ are also closely tied to results of Bridgeman $[22,23]$ on bending of surfaces in hyperbolic spaces. Here we shall give a simple conceptual proof without an explicit estimate. The number of bending geodesics that 
hit the $r$-ball has no uniform bound (if it did the lemma would be trivial since every crescent has angle $\leq 2 \pi$ ). However, the total bending of these geodesics is bounded in terms of $r$. This result (together with Lemma 31) is one of the main pillars on which the whole paper rests; the uniform estimate of bending eventually becomes the uniform estimates of time and accuracy given in Theorems 1 and 2.

Lemma 9 There is a $C<\infty$ so that $B(r) \leq C e^{3 r}$.

Proof Suppose $\Omega$ is normalized so $\infty \notin \Omega$. The normalization implies that if $\gamma$ is a bending geodesic in $\mathbb{H}^{3}$ that hits the plane at 1 and -1 , then the corresponding crescent is in the unit disk. Moreover, an easy estimate shows that a crescent with vertices \pm 1 and angle $\alpha$ has area $\geq c \alpha$ for some fixed $c>0$.

If $\tilde{\gamma}$ is a bending geodesic with angle $\beta$ that passes within hyperbolic distance $r$ of $(0,0,1)$ then the "highest" point of $\tilde{\gamma}$ has Euclidean height at least $e^{-r}$ above the plane $\mathbb{R}^{2}$. Thus its two endpoints on the plane are at least $2 \cdot e^{-r}$ apart. Moreover, at least one endpoint must be contained in the disk of diameter $e^{r}$ around the origin (if not, then $\tilde{\gamma}$ lies outside the hemisphere with this disk as its base, which means the hyperbolic distance to $(1,0,0)$ is $\geq r)$.

Thus the part of the crescent corresponding to $\tilde{\gamma}$ inside the ball $B\left(0, e^{r}+1\right)$ has area at least $c e^{-r} \beta$. Consider the set of all bending geodesics that come within hyperbolic distance $r$ of the point $(0,0,1) \in \mathbb{H}^{3}$ and let $\left\{\alpha_{n}\right\}$ be an enumeration of the bending angles. Since the crescents are disjoint we deduce $\sum_{n} \alpha_{n} \leq \frac{1}{c} \pi e^{r}\left(e^{r}+1\right)^{2} \leq$ $C e^{3 r}$, as desired. (Note that this argument is not sharp since the crescents can have small area only when then are close to the origin.)

The following simple lemma quantifies the fact that an elliptic Möbius transformation with small rotation angle is close to the identity.

Lemma 10 Suppose $\sigma$ is an elliptic Möbius transformation with fixed points $a, b$ and rotation angle $\theta$. If $r=\max (|z-a|,|z-b|) \leq A|b-a| \leq|b-a| /(4 \theta)$ and $|\theta| \leq \frac{1}{4}$, then we have

$$
|z-\sigma(z)| \leq 2 A^{2}|\theta||z-a|
$$

where $C$ depends only on $A$.

Proof This is an explicit computation. The conclusion is invariant under scaling, so we may assume $a=1, b=-1$, in which case $\sigma$ has the form $\sigma(z)=\tau^{-1}(\lambda \tau(z))$ where $\lambda=e^{i \theta}$ and $\tau(z)=(z-1) /(z+1)$. Doing some arithmetic, and using $|1-\lambda| \leq$ $|\theta|$, we get

$$
|\sigma(z)-z|=\left|\frac{(1-\lambda)-(1-\lambda) z^{2}}{(1+\lambda)+(1-\lambda) z}\right| \leq|\theta| \frac{\left|1-z^{2}\right|}{1-|\theta|-|\theta||z|} \leq 2 A^{2}|\theta||z-1|,
$$

if $|\theta| \leq \frac{1}{4}$ and $|\theta z| \leq \frac{1}{4}$.

The following is the main result of this section. Recall that $R: \Omega \rightarrow S$ denotes the nearest point retraction discussed in the previous section. 
Lemma 11 Suppose $r>0$ is given. There is an $\epsilon>0$, depending only on $r$, so that if $0 \leq s<t \leq 1$ and $|s-t| \leq \epsilon$ then the following holds. Suppose $G_{1}$ and $G_{2}$ are gaps in the normal crescent decomposition of the finitely bent domain $\Omega_{s}$ such that $\rho_{S}\left(R\left(G_{1}\right), R\left(G_{2}\right)\right) \leq r$. Suppose $\tau_{j}$ are Möbius transformations so that $\left.\varphi_{s, t}^{-1}\right|_{G_{j}}=\tau_{j}$ for $j=1,2$. Then

$$
\rho_{t}\left(\tau_{1}(z), \tau_{2}(z)\right) \leq C_{r}|t-s|,
$$

for every $z \in \Omega_{s}$ with $\rho_{s}\left(z, G_{1}\right) \leq r$.

Proof The statement is invariant under renormalizing by Möbius transformations so we may assume that $G_{1}$ has base disk $\mathbb{D}$, that $z_{1}=0 \in G_{1}$ is within $2 r$ of $G_{2}$, and that $\tau_{1}$ is the identity.

Then $\tau_{2}$ is a composition of the elliptic transformations $\left\{\sigma_{j}\right\}$ that correspond to the normal crescents $\left\{C_{j}\right\}$ that separate $G_{1}$ and $G_{2}$. By Lemma 9, the measure of the bending geodesics separating $G_{1}$ and $G_{2}$ is at most $B(r)$.

Since $\rho_{S}\left(C_{j}, 0\right) \leq r$ for all $j, C_{j}$ has diameter $\geq e^{-r}$ and one vertex is contained within $D\left(0, e^{r}\right)$ by the proof of Lemma 9. By Lemma 10, this means that $\sigma_{j}$ moves points in $D(0, C)$ at most $C\left|\theta_{j}\right|$ with $C$ depending only on $r$, assuming $\theta_{j}$ is small enough (depending only on $r$ ). Thus

$$
\left|\tau_{2}(z)-z\right| \leq C_{r}|s-t| \sum_{j}\left|\theta_{j}\right|=O(|s-t|),
$$

for $|z| \leq C$, assuming $|s-t|$ is small enough, depending only on $r$.

If $\rho_{t}(0, z) \leq r$, then $|z| \leq A_{r}$ and $\operatorname{dist}\left(z, \partial \Omega_{s}\right) \geq B_{r}>0$ with estimates that only depend on $r$ (see Lemma 42, Appendix A). Thus for $|s-t|$ small enough, $\rho_{t}(z, 0) \leq r$ and $|z-w| \leq \epsilon$ imply $\rho_{t}(z, w) \leq C_{r} \epsilon$. Hence for a given $r$ we can choose $|s-t|$ so small that (1) implies $\rho_{t}\left(\tau_{2}(z), z\right) \leq O(|s-t|)$ (with constant depending on $r$ ).

Lemma $12 \varphi_{s, t}$ is a quasi-isometry with constant $O(|s-t|)$.

This follows immediately from the following technical result that will be proven in Sect. 7. It also follows from a careful reading of [14], which gives an explicit construction of a quasiconformal map from $\mathbb{D}$ to a finitely bent domain $\Omega$ with boundary values $\varphi^{-1}$. The method can be adapted to give an explicit map $\Omega_{s} \rightarrow \Omega_{t}$ that is quasiconformal with constant $O(|s-t|)$.

Theorem 13 Suppose $\Omega_{0}, \Omega_{1}$ are simply connected and $\varphi: \Omega_{0} \rightarrow \Omega_{1}$ has the following property: there is a $0<C<\infty$ so that given any hyperbolic $C$-ball $B$ in $\Omega_{0}$, there is a Möbius transformation $\sigma$ so that $\rho_{\Omega_{0}}(z, \sigma(\varphi(z))) \leq \epsilon$ for every $z \in B$. Then there is a hyperbolic $(1+O(\epsilon))$-biLipschitz map $\psi: \Omega_{0} \rightarrow \Omega_{1}$ so that $\sup _{z \in \Omega_{0}} \rho_{\Omega_{1}}(\varphi(z), \psi(z)) \leq O(\epsilon)$. In particular, $\varphi$ is a quasi-isometry between the hyperbolic metrics with constant $O(\epsilon)$.

Corollary 14 There is a (hyperbolically) $(1+O(|s-t|))$-biLipschitz map $\psi_{s, t}$ : $\Omega_{s} \rightarrow \Omega_{t}$ so that $\psi_{s, t}=\varphi_{s, t}^{-1}$ on the boundary. If $G$ is a gap or crescent and $\varphi_{s, t}^{-1}$ is the Möbius transform $\sigma$ on $G$, then $\rho_{t}\left(\psi_{s, t}(z), \sigma(z)\right) \leq O(|s-t|)$ for $z \in G$. 


\section{Piecewise Möbius Maps and $\epsilon$-Delaunay Triangulations}

Here we prove Theorem 13 from Sect. 6.

If we want to approximate a map $f$ between polygons, a convenient thing to do is to decompose the interior into triangles, and approximate by a map that is linear on each triangle. If $f$ is already linear in some subregion, we can arrange for the approximation to agree with it on the triangles that lie inside this subregion.

We would like to do the same thing for finitely bent domains. One problem is that the maps we wish to approximate are Möbius in some regions rather than linear, and a piecewise linear approximation will not preserve this. We could try to approximate circular arcs by line segments and Möbius transformations by linear maps, but instead we will slightly alter the idea of piecewise linear approximation.

Given a triangle $T$, let $D$ be the disk containing the three vertices on its boundary and let $\tilde{T}$ be the ideal hyperbolic triangle in $D$ with these three vertices. We will say that a triangulation is $\epsilon$-Delaunay if whenever two Euclidean triangles $T_{1}, T_{2}$ meet along an edge $e$, the sum of two the angles not incident on $e$ is at most $\pi-\epsilon$. This means that between $\tilde{T}_{1}$ and $\tilde{T}_{2}$ there is a crescent of angle at least $\epsilon$; see Fig. 27. A 0-Delaunay triangulation is the same as the usual notion of a Delaunay triangulation. Delaunay triangulations play an important role in computational geometry (see, e.g., $[8,60,61,106])$.

Lemma $15 \epsilon$-Delaunay triangulations are invariant under Möbius transformations.

Proof The $\epsilon$-Delaunay condition is equivalent to saying that if $T_{1}$ and $T_{2}$ are adjacent triangles then the boundaries of the corresponding disks $D_{1}$ and $D_{2}$ meet at exterior angle less than $\pi-\epsilon$. This is clearly invariant under Möbius transformations.

Suppose we are given a mapping between the boundaries of two crescents with interior angles $\alpha_{1}, \alpha_{2}$ that agrees with a Möbius transformation on each boundary arc (but possible different transformations on each arc). Normalizing so the vertices are 0 and $\infty$, the boundary maps must be of the form $z \rightarrow \lambda_{i} z$ for $i=1,2$. Mapping the crescents to strips $S_{i}=\left\{z=x+i y: 0 \leq y \leq \alpha_{i}\right\}$ by a logarithm, these maps become $z \rightarrow z+t_{i}$. The boundary map can be extended to the interior by a unique affine map $T:(x, y) \rightarrow\left(x+t_{1}+\left(t_{2}-t_{1}\right) y / \alpha_{1}, y \frac{\alpha_{2}}{\alpha_{1}}\right)$. When this map is conjugated back to a map between the crescents, it defines a quasi-conformal map with minimal possible dilatation extending the given boundary values (e.g., Theorem 3.1 of [56]

Fig. 27 Two triangles share an edge and the angles opposite the edge sum to less than $\pi$. There is then a crescent of angle $\pi-(\alpha+\beta)$ that separates the ideal hyperbolic triangles associated the two Euclidean triangles

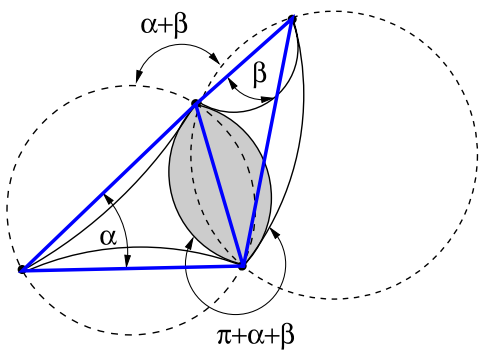


for a simple proof; strict equality actual holds $[9,120])$. We shall call such a map an affine-crescent map.

Suppose we are given an $\epsilon$-Delaunay triangulation in a region $\Omega$ and a map $f: \Omega \rightarrow \Omega^{\prime}$ that sends the vertices to the vertices of another $\epsilon$-Delaunay triangulation. On each $\tilde{T}$, define $g$ to be the Möbius transformation defined by the images of the three vertices. On the crescents separating two ideal triangles, define $g$ to be the affine crescent map extending the definition on the boundary of the crescent. Thus $g$ is an approximation to $f$ that is Möbius on the ideal triangles and quasiconformal on the crescents. If $f$ is Möbius on the quadrilateral formed by two adjacent triangles, then it is $g=f$ on the two corresponding ideal triangles and the crescent separating them. Otherwise the quasiconformal constant of $g$ is bounded in terms of the quasiconformal constant of $f$ and the hyperbolic size of the triangles.

We will call an (infinite) $\epsilon$-Delaunay triangulation an $(\epsilon, s)$-triangulation for $\Omega$ if every edge has hyperbolic diameter $\sim s$ in $\Omega$ and the circumcircle of every triangle has hyperbolic diameter $\sim s$. Next we want to observe that such a triangulation always exists.

The plane can be tiled by a collection equilateral triangles $\mathcal{T}_{n}$ of side length $2^{-n}$ in such a way that the each triangle of size $2^{-n}$ is a union of four triangles in $\mathcal{T}_{n+1}$. Given a point $x \subset \Omega$ and $0<\lambda<\frac{1}{4}$ there is a triangle $T \in \mathcal{T}_{n}$ that contains $x$ and so that

$$
(\lambda / 2) \operatorname{dist}(T, \partial \Omega) \leq \ell(T) \leq \lambda \operatorname{dist}(T, \partial \Omega)
$$

and it is unique except when $x$ is on the common boundary of a finite number $(\leq 6)$ of such triangles. Any two triangles that satisfy this condition have adjacent sizes (since they are both comparable to the same number within a factor of two).

So we can cover $\Omega$ by a union of triangles whose interiors are disjoint and each is approximately size $\lambda$ in the hyperbolic metric. We claim that by adding some extra edges we can preserve this property and also get a $\epsilon$-Delaunay triangulation. To see how, form a triangular mesh by taking the lattice triangles whose size is comparable to the distance to the boundary.

We can also arrange that if two triangles meet a common triangle, then they must be of adjacent sizes. To see this, suppose $T_{1}$ and $T_{2}$ are adjacent and $T_{2}$ and $T_{3}$ are adjacent and that $T_{1}$ is the largest of the three triangles. Let $\ell(T)$ denote the side length of a equilateral triangle. Then

$$
\begin{aligned}
\ell\left(T_{3}\right) & \geq(\lambda / 2 \sqrt{2}) \operatorname{dist}\left(T_{3}, \partial \Omega\right) \\
& \geq(\lambda / 2 \sqrt{2})\left[\operatorname{dist}\left(T_{1}, \partial \Omega\right)-\ell\left(T_{2}\right)-\ell\left(T_{3}\right)\right] \\
& \geq(\lambda / 2 \sqrt{2})\left(\frac{1}{\lambda}-1-1\right) \ell\left(T_{1}\right) \\
& \geq\left(\frac{1}{2 \sqrt{2}}-\frac{\lambda}{\sqrt{2}}\right) \ell\left(T_{1}\right) \\
& >\frac{1}{4} \ell\left(T_{1}\right),
\end{aligned}
$$


Fig. 28 When triangles of different size meet we subdivide the larger one to make a triangulation. This produces $\epsilon$-Delaunay triangulations for a uniform $\epsilon>0$

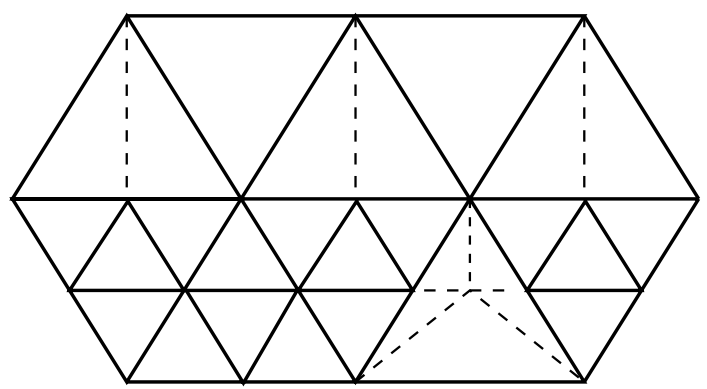

if $\lambda$ is small enough. Since $\ell\left(T_{1}\right) / \ell\left(T_{3}\right)$ is a power of 2 we must have $\ell\left(T_{3}\right) \geq$ $\ell\left(T_{1}\right) / 2$, as desired.

If two adjacent triangles are different sizes then some interior edges must be added to the larger one to make it a triangulation (but the smaller one does not hit an even smaller one by our previous calculation, so it does not need to be divided). There are three cases:

(1) If the larger one is bordered on all three sides by smaller ones, then we divide it into four equilateral triangles in the usual way.

(2) If is bounded on exactly one side by smaller triangles, we add the bisector of the opposite angle.

(3) If it is bounded on exactly two sides by smaller triangles, we add the segment parallel to the third side $e$ and half its length and the three segments connecting the midpoint of the new segment to corners of the triangle.

See Fig. 28. This is clearly $\epsilon$-Delaunay. Indeed the worse case is in the third case above. The bottom triangle has two angles of size $\alpha=\arctan (\sqrt{3} / 2) \approx 0.713714 \approx$ $0.22718 \pi$ and one of angle $\beta=(\pi-2 \alpha) \approx 0.544 \pi$. Since $\alpha$ is opposite an angle of size $\frac{2}{3} \pi$ and $\beta$ is opposite angle of $\frac{1}{3} \pi$, we see that every possible quadrilateral is at least $0.106 \pi$-Delaunay.

The proof of Theorem 13 is now quite simple. Take a $\left(\epsilon_{0}, s\right)$-triangulation of $\Omega_{1}$, restrict the map $\varphi$ to the vertices and take $\psi$ to the piecewise Möbius extension of these values to $\Omega_{1}$. If $s$ is smaller than $C / 2$ then on the union of any two adjacent triangles, the map $\varphi$ is $\epsilon$-close to a Möbius transformation, and this implies $\psi$ is hyperbolic biLipschitz with constant $1+O(\epsilon)$ where the constant depends only on $\epsilon_{0}$ and $s$. This proves Theorem 13.

\section{Computing the Bending Lamination in Linear Time}

We have now finished introducing the $\iota$ map and describing the relevant estimates. We now start our discussion of the algorithm for computing conformal maps, starting with the construction of the bending lamination of a finitely bent domain in linear time. This will lead to our decomposition of the plane, the representation of conformal maps and the method for improving such representations.

Recall that $R$ denotes the nearest point retraction from a planar domain to its dome. Given a finitely bent domain $\Omega$, we noted above that $\varphi=\iota \circ R$ is a continuous map 
Fig. 29 The convex hull of six points, a lamination with these endpoints (solid lines) and a completion of it (dashed lines)

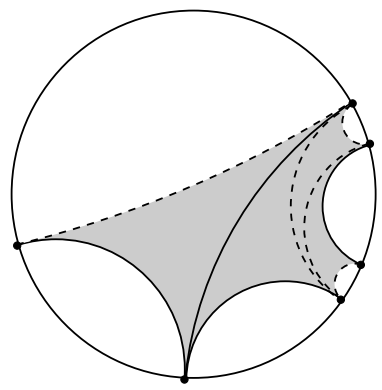

of $\Omega$ to $\mathbb{D}$, equals $\iota$ on the boundary, is a Möbius transformation on each gap, and collapses the crescents to a union of geodesics $\Gamma$ in $\mathbb{D}$ called the bending lamination. To each geodesic $\gamma \in \Gamma$ we associate the angle of the corresponding crescent. This is called the bending measure of the geodesic and is an example of a transverse measure on a lamination.

A finite lamination $\Gamma$ in the disk lies in the hyperbolic convex hull of its endpoints. If it triangulates the convex hull we say it is complete. We shall assume that our bending laminations are complete, which is always possible by adding at most $O(n)$ extra geodesics with bending angle 0 (since we only need $2 n-3$ edges to triangulate $n$ points). See Fig. 29.

Next we will check that $\iota$ and the bending lamination of a finitely bent domain $\Omega$ can be constructed in linear time, given the medial axis of $\Omega$. This is fairly straightforward, but we record it formally with some definitions and a lemma. Suppose we have a finite collection of disks, $\mathcal{D}$, in the plane and an adjacency relation between them that makes the collection into the vertices of a tree. Suppose the disk $D_{0}$ has been designated the root of the tree. Then any other disk $D$ has a unique "parent" $D^{*}$ that is adjacent to $D$ but closer to the root. Assume that for every (non-root) disk we are given a map $\tau_{D}: D \rightarrow D^{*}$. Then we can define a map $\sigma_{D}: D \rightarrow D_{0}$ as follows. If $D=D_{0}$, the map is the identity. Otherwise, there is a unique shortest path of disks $D_{0}, \ldots, D_{k}=D$ between $D_{0}$ and $D$. Note that each disk is preceded by its parent. Thus $\sigma=\tau_{D_{1}} \circ \cdots \circ \tau_{D_{k}}$ is a mapping from $D$ to $D_{0}$ as desired. We will refer to this as a "tree-of-disks" map.

Lemma 16 With notation as above, assume that every map $\tau_{D}$ is Möbius. Then given $n$ points $\mathbf{v}=\left\{v_{1}, \ldots, v_{n}\right\}$, with $v_{k} \in \partial D_{k}$ for $k=1, \ldots, n$, we can compute the $n$ image points $\sigma(\mathbf{v}) \subset \partial D_{0}$ in at most $O(n)$ steps.

Proof If $D \in \mathcal{D}$ has positive radius, choose three distinct reference points $z_{1}^{D}, z_{2}^{D}, z_{3}^{D}$ on $\partial D$; otherwise let this collection be empty. Every other point $z$ on $\partial D$ is uniquely determined by the cross-ratio $\operatorname{cr}\left(z_{1}^{D}, z_{2}^{D}, z_{3}^{D}, z\right)$. Label each point $v$ in $\mathbf{v}$ with the minimal $k$ so that $v$ is on the boundary of a $k$ th generation disk $D$. For $k=0$, we do nothing to the vertex. For $k>0$, compute $\tau_{D}(v) \in \partial D^{*}$ and record the cross-ratio $\operatorname{cr}\left(z_{1}^{D^{*}}, z_{2}^{D^{*}}, z_{3}^{D^{*}}, \tau_{D}(v)\right)$. Also compute and record the images of the three reference points for $D$, i.e., $\operatorname{cr}\left(z_{1}^{D^{*}}, z_{2}^{D^{*}}, z_{3}^{D^{*}}, \tau_{D}\left(z_{k}^{D}\right)\right)$ for $k=1,2,3$.

If a vertex is on $\partial D_{0}$ then it maps to itself. If $D$ is a first generation disk, then we just compute $\tau_{D}(v) \in \partial D_{0}$ and compute the $\tau_{D}$ images of the three reference 
points for $D$. For each child of $D^{\prime}$ of $D$, we can now compute $\sigma_{D^{\prime}}$ for any associated vertices using the previously recorded cross ratios with respect to the reference points for $D$ and we can also compute the images on $\partial D_{0}$ for the references points for $D^{\prime}$. In general, if $D$ is a disk and we have already computed where the reference points for its parent are mapped on $\partial D_{0}$, we can use the recorded cross-ratio information to compute where the associated vertices and reference points for $D$ map to. This allows us to map every point of $\mathbf{v}$ to $\partial D_{0}$ in $O(n)$ steps.

To construct the bending lamination of a finitely bent domain we apply this lemma to the collection of base disks corresponding to the gaps of the normal crescent decomposition and with two gaps being adjacent iff they are separated by a single crescent. In this case, adjacent disks either (1) intersect at exactly two points and we take the elliptic transformation that fixes these points and moves the child to the parent, or (2) the disks coincide (if the crescent had bending angle 0) and we take the identity map. We can also compute the gaps and the Möbius transformations mapping these gaps to the ones in $\Omega$ in time $O(n)$.

In general, the disks in a "tree-of-disks" need not intersect. In [17], this lemma is used to construct the exact $\iota$ map for a polygon. The vertices are the medial axis disks corresponding to the vertices of the medial axis with adjacency inherited from the medial axis. Adjacent disks need not intersect (e.g., consider two ends of a long edgeedge bisector), but we can still define an explicit Möbius transformation between them (but not an elliptic transformation in this case).

\section{Covering the Bending Lamination}

Our goal in this section is to cover the bending lamination of a finitely bent domain by standard regions. Our standard regions will be "Whitney boxes", which are approximately unit hyperbolic neighborhoods of points, and "Carleson towers", which look like unit neighborhoods of long hyperbolic geodesic segments.

The construction can carried out either in the unit disk or the upper half-plane. It is slightly easier to draw accurate figures in the upper half-plane, so we will describe it there, and only trivial changes are needed to move it to the unit disk.

Given an interval $I \subset \mathbb{R}$, the corresponding Carleson square is the region in the upper half-plane of the form $\{z=x+i y: x \in I, 0<y<|I|\}$. The "top-half" of $Q$ is $T(Q)=\{z \in Q: y>|I| / 2\}$. This will be called a Whitney box, and its Euclidean diameter is comparable to its Euclidean distance from $\mathbb{R}$ (abusing notation we may also call them Whitney "squares", even though they are Euclidean rectangles; the main point is that they are approximately unit size in the hyperbolic metric). When $I$ ranges over all dyadic intervals (i.e., all intervals of the form $\left.\left[j 2^{-n},(j+1) 2^{-n}\right]\right)$, the corresponding Whitney boxes partition the upper half-plane into pieces with approximately unit hyperbolic size; see Fig. 30. Carleson squares are named after Lennart Carleson who used them in his solution of the corona problem and they are now ubiquitous in function theory [27, 64].

Dyadic Carleson squares form a tree under intersection of the interiors. Each square has a unique parent and two children. The parent of a dyadic Carleson square 
Fig. 30 A decomposition of a Carleson square into dyadic Whitney boxes [64]
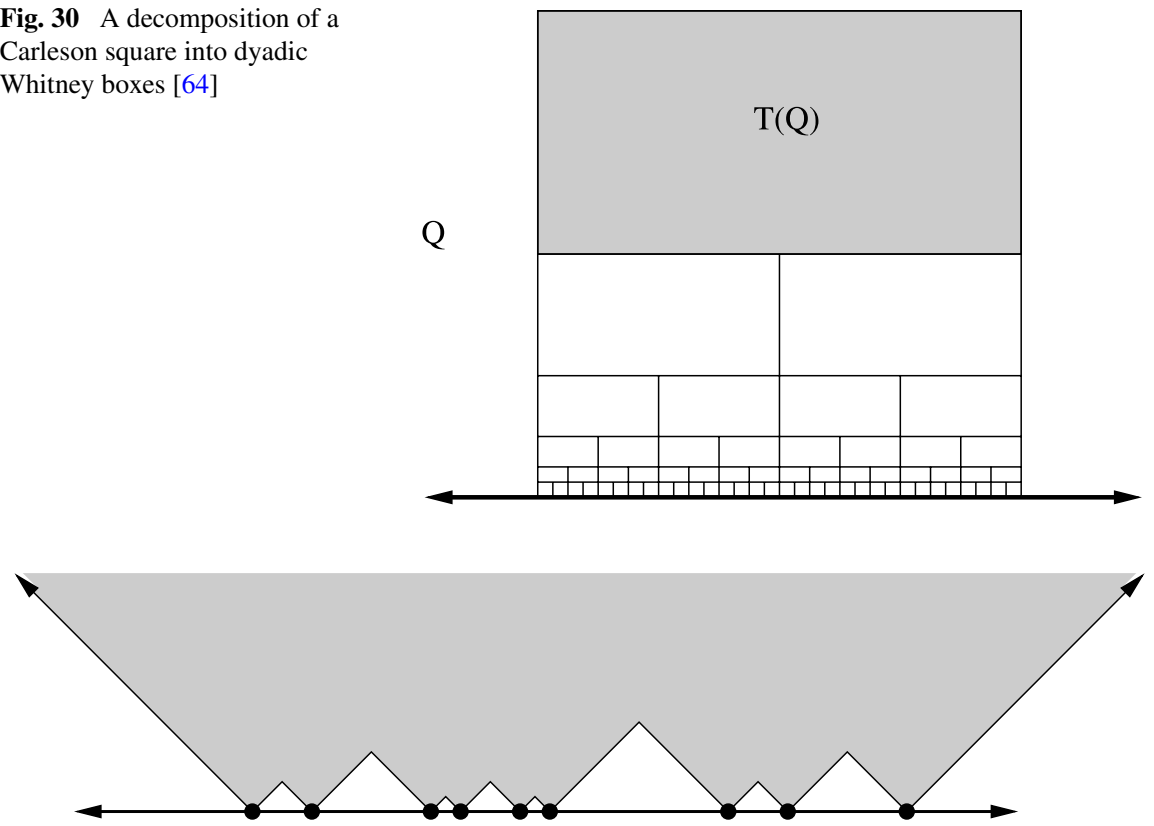

Fig. 31 The sawtooth domain associated to the set $S$. This region is approximately a unit neighborhood of the bending lamination and has only $O(n)$ boundary arcs

$Q$ will be denoted $Q^{*}$. This obviously also induces a tree structure on Whitney boxes. We will say two dyadic Whitney boxes are neighbors if they are the same size and adjacent; each box therefore has a "left" and a "right" neighbor. One of these is a "sibling" in the sense that it shares a parent, while the other does not.

Suppose $\Gamma$ is a complete, finite geodesic lamination in $\mathbb{H}$. We assume that $\Gamma$ has been normalized so that its set of endpoints $S$ satisfies $\{0,1\} \subset S \subset[0,1]$.

For $x \in \mathbb{R}$, let $W(x) \subset \mathbb{H}$ be the Euclidean cone of angle $\pi / 2$ and vertex $x$ whose axis is vertical. This is called the Stolz cone with vertex $x$. Then $W=\bigcup_{x \in S} W(x)$ is an infinite polygon with $2 n$ sides; see Fig. 31 . This type of region is called a sawtooth domain and is also approximately a unit neighborhood of $\Gamma$ (at least if we truncate it at height 2). Clearly we can compute $W$ from $S$ in linear time if we are given $S$ as an ordered set.

We can compute the medial axis of $W$ in time $O(n)$ (we don't even need the full strength of the theorem of Choi-Snoeyink-Wang: $W$ is a monotone histogram so a modification of the simpler algorithm from [1] for convex polygons will work in linear time, see [33]). In the medial axis of $W$, an edge-edge bisector must be vertical. Suppose $A_{0}$ is a large number (to be fixed later, but $A_{0}=20$ will work) and consider an edge-edge bisector $e$ of hyperbolic length $\geq A_{0}$.

First, suppose $e$ has finite hyperbolic length. This simply means that $e$ is bounded away from $\infty$ and the real line (i.e., it is a compact subset of $\mathbb{H}$ ). Let $Q_{1}, Q_{2}$ denote the Whitney boxes that contain the top and bottom endpoints of $e$, respectively. These must be distinct because we are assuming $e$ has large length, so its endpoints are in boxes far apart. Let $e^{*}$ and $Q_{1}^{*}$ denote the vertical projections of $e$ and $Q_{1}$ onto the 


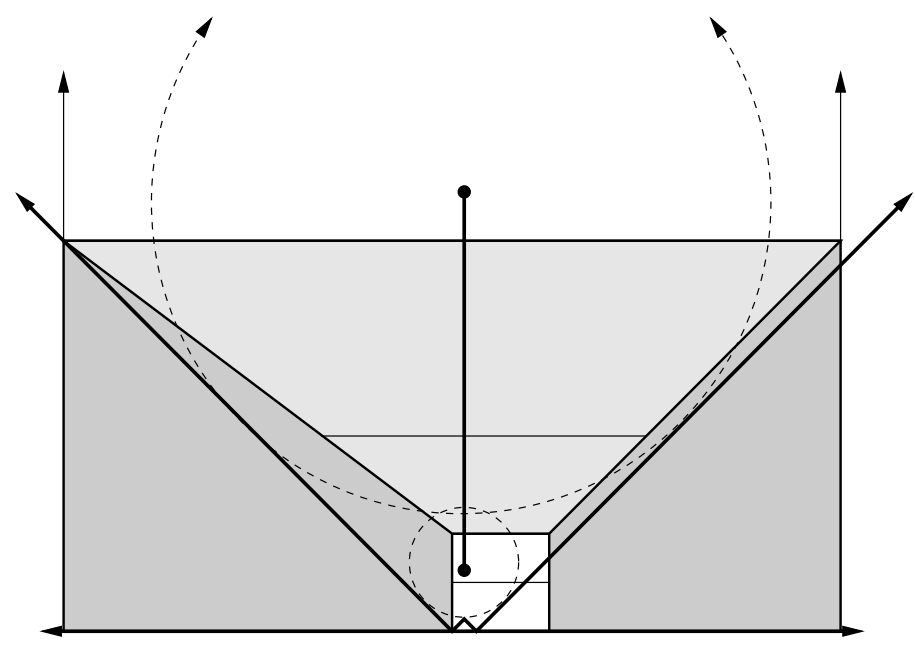

Fig. 32 The union of shaded areas is a Carleson arch. The lighter shaded trapezoid is the corresponding Carleson tower

real axis. If $e^{*}$ is contained in the middle third of $Q_{1}^{*}$ then let $Q_{3}=Q_{1}$. If $e^{*}$ is in the left third of $Q_{1}^{*}$ then let $Q_{3}$ denote the union of $Q_{1}$ and its neighbor to the left. If $e^{*}$ is in the right third, we take the union with the neighbor to the right. In every case, $Q_{2}^{*}$ now hits the middle third of $Q_{3}^{*}$.

The corresponding Carleson tower is the trapezoid that has the bottom edge of $Q_{3}$ and the top edge of $Q_{2}$ as its bases; see Fig. 32. Each tower is associated to a unique edge of the medial axis so obviously there are at most $O(n)$ towers associated to a set $S$ with $n$ points. The corresponding Carleson arch is $Q_{4} \backslash Q_{5}$ where $Q_{4}$ is the union of Carleson squares with top edge contained in the bottom edge of $Q_{3}$ and $Q_{5}$ is the Carleson square whose top edge is the top edge of $Q_{2}$.

The decomposition piece associated to $e$ is $Q=Q_{3} \backslash Q_{2}$ and is called an arch. It meets the real line in two intervals since the base of $Q_{2}$ is much shorter that the base of $Q_{3}$ and hits the center third of $Q_{3}$ 's base. If $e$ has hyperbolic length $L$ then the resulting arch is said to have hyperbolic "width" $L$ and the extremal distance in $\mathbb{H}$ between the two components of $Q \cap \mathbb{R}$ is $\frac{\pi}{L}+O(1)$. We will call this an $\epsilon$-arch if $\epsilon \geq \pi / L$. Note that if $\epsilon$ is small, then the two boundary components of a $\epsilon$-arch in $\mathbb{H}$ in $\mathbb{H}$ are very far apart in the hyperbolic metric $(\sim 1 / \epsilon)$.

If $e$ has infinite length then the corresponding Carleson tower is a triangle with one vertex on $\mathbb{R}$ where $e$ hits the line, and opposite side that is the bottom of $Q_{3}$ (defined as above). The Carleson arch is "degenerate" in this case: it is the same as the union of Carleson squares $Q_{4}$ above, except that it comes with a special marked point in the middle third of its base.

Also note that any two hyperbolic geodesics connecting the top and bottom edges of the corresponding Carleson tower must be within $O\left(e^{-d}\right)$ of each other when they are more than hyperbolic distance $d$ from either arch boundary. Thus the convex hull is thin in the arches, in the sense that removing a small ball there will disconnect it. This is not true outside the arches. 
If we are given $A>A_{0}$ then we let $e^{\prime}$ denote the "central part" of $e$, i.e., the points of $e$ that are at least hyperbolic distance $A$ from the endpoints of $e$. Associated to $e^{\prime}$ is a Carleson arch called the "central arch" or " $A$-arch" associated to $e$. Let $\left\{e_{j}\right\}$ be an enumeration of the edge-edge bisectors of length $\geq A_{0}$ in the medial axis of $W$, let $A_{j}$ be the Carleson arch associated to $e_{j}$ and $A_{j}^{\prime}$ the arch associated to its central part $e_{j}^{\prime}$ (possibly empty if $e$ is shorter than $2 A$ ). Let $\mathcal{K}$ be an enumeration of the connected components of $\mathbb{H} \backslash \bigcup_{j} A_{j}^{\prime}$ and let $\mathcal{N}$ be the $A_{0}$-arches. ( $\mathcal{N}$ is for "thin" and $\mathcal{K}$ is for "thick".) Then the sets in $\mathcal{N}$ and $\mathcal{K}$ cover the whole upper half-plane and each set only overlaps sets of the other type. These overlaps are themselves Carleson arches corresponding to segments of hyperbolic length $A-A_{0}$. This covering of $\mathbb{H}$ will be referred to as a thick/thin decomposition of $\mathbb{H}$ with $\eta$-thin overlaps, where $\eta^{-1} \approx A-A_{0}$. We will use it later to define a thick/thin decomposition of a polygon in Sect. 12.

Lemma 17 The number of Whitney boxes that hit $C(S)$ but do not hit any central Carleson arch is at most $O(A n)$.

Proof First, suppose $A=A_{0}$. Since $\Gamma$ is complete and contains $n$ geodesics, its hyperbolic convex hull can be split into $n-2$ ideal hyperbolic triangles and hence the hyperbolic convex hull has hyperbolic area bounded by $\pi(n-2)$; see Appendix A for a discussion of hyperbolic area. Thus it is enough to show that there is a $\delta>0$ and a $C<\infty$ so that each Whitney box $Q$ can be associated to a hyperbolic ball of area $\delta$ contained in the convex hull of $\Gamma$ and within hyperbolic distance $C$ of $Q$. For, if we place $M$ balls of area $\delta$ into a set of area $\leq \pi n$, then at least $M \delta / \pi n$ of them intersect in a common point. Since any point can only have a bounded number of distinct Whitney boxes within hyperbolic distance $C$ of it, we see that $M$ must be bounded by a multiple of $n$ (the constant depending on $\delta$ and $C$ ). If $Q$ is a Whitney box that hits $C(S)$, but does not hit any $\epsilon$-Carleson arch, then either it is about unit size (there are only $O(1)$ such boxes hitting $C(S))$ or $2 Q \cap C(S)$ contains a ball with radius bounded below uniformly, which completes the proof.

If $A>A_{0}$ then the gap between and $A_{0}$-arch and its central $A$-arch can be filled by $O(A)$ Whitney boxes. Since there are $O(n)$ arches, this is at most $O(A n)$ extra boxes.

Thus we know that given $n$ ordered points in $\mathbb{R}$ the convex hull of these points can be covered by $O(n)$ Carleson towers and $O(n)$ Whitney boxes. Next we wish to show these towers and boxes can actually be found in time $O(n)$.

Lemma 18 Suppose $\Gamma$ is a finite, complete geodesic lamination containing $n$ geodesics. Then in time $O(n)$ we can find a covering of $\Gamma$ by at most $O(n)$ dyadic Whitney boxes and Carleson towers.

Proof Compute the medial axis of the sawtooth domain $W$ corresponding to the set of endpoints $S$. Locate all the edge-edge bisectors of hyperbolic length $\geq A_{0}$ and record the associated Carleson towers. 


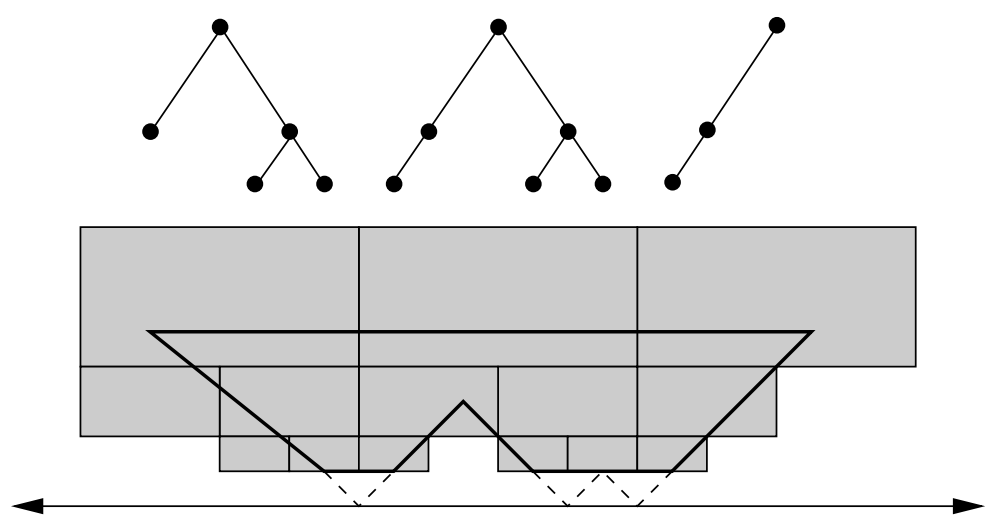

Fig. 33 A truncated component of $W$ and the dyadic Whitney boxes that hit it. These boxes form vertices of at most three trees rooted at the topmost boxes

Each tower has a top and bottom horizontal edge. Extend these edges until they hit the boundary of $W$ (recall that we know which edges of $W$ get hit). Near a tower, $W$ looks very close to a Stolz cone, so the length of these edges is close to 1 if $A_{0}$ is large enough. In particular, for large $A_{0}$ it has length $<2$ and so at most three Whitney boxes suffice to cover each of these edges. (This determines our choice of $A_{0}$.)

These edges cut $W$ into connected components. After we remove the components corresponding to towers, we have at most $O(n)$ components remaining. We claim that if one of these components hits $m$ dyadic Whitney boxes then we can find all these boxes in time $O(m)$.

To do so consider one such component $W_{0}$. It has a single horizontal "top" edge of length $L$ and a bottom edge that is polygonal curve. Project each segment of this curve vertically to the real line and choose a dyadic interval contained in each projection and of comparable size. Then enumerate all the dyadic intervals which contain at least one of our choice and have length $\leq L$. If there are $m$ such they can be listed in time $O(m)$ (see Lemma 50 of Appendix A). The corresponding Whitney boxes, plus a constant number of their neighbors, cover $W_{0}$, so we are done. See Fig. 33.

As a consequence of the proof, we see that we can also record the adjacency relations between the chosen boxes in time $O(n)$.

\section{Extending the Convex Hull Cover to All of $\mathbb{H}$}

Given a finite set of points $S \subset[-1,1]$ we will construct the corresponding "Carleson-Whitney" decomposition of the upper half-plane by modifying the cover of the convex hyperbolic hull of $S$ from Sect. 9. This decomposition of the upper half-plane will consist of the outside of some fixed Carleson square $Q_{0}$ and a finite number of pieces whose union is all of $Q_{0}$. These pieces come in four types (see Figs. 34 and 35): 


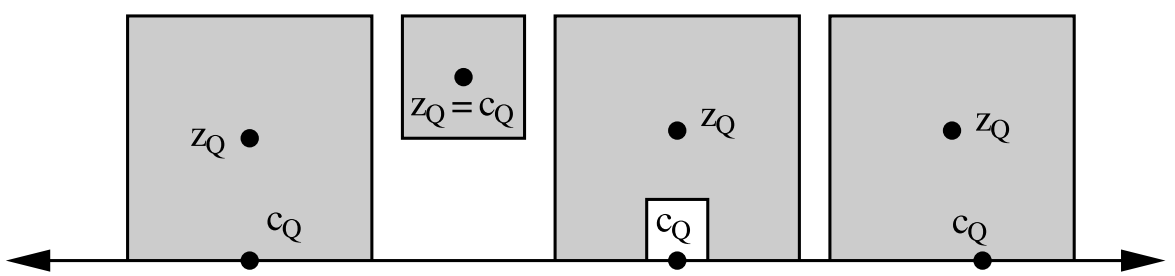

Fig. 34 The shapes of pieces used in a Carleson-Whitney decomposition: Carleson squares, Whitney squares, Carleson arches and degenerate Carleson arches

Fig. 35 Definition of a Carleson arch. The bounding squares of an arch need not be dyadic, in which case we tile the region around them by Whitney boxes and Carleson squares to fill in a union of dyadic squares

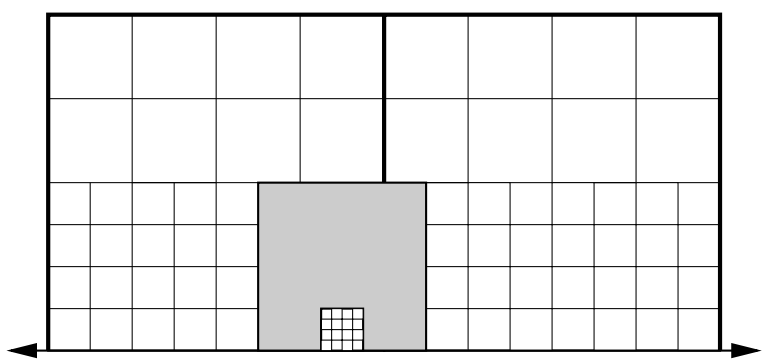

(1) Carleson squares.

(2) Whitney squares, which are obtained by dividing a Whitney box into a bounded number of equal sized Euclidean squares.

(3) Carleson arches, which consist of a Carleson square with a smaller Carleson square removed from it; we assume the base of the smaller square hits the middle third of the base of the larger square and is much smaller.

(4) Degenerate arches, where the smaller square is replaced by a single point of $S$, contained in the center third of the base. Moreover, every point of $S$ will be associated to a degenerate arch in this way. Note that these pieces are really Carleson squares, but it is convenient to consider them separately from squares $Q$ whose closures do not contain a point of $S$.

In practice, we could avoid arches by simply using more Whitney type squares but we would lose the linear dependence on $n$. For many domains, however, this might not be much of a loss, and would simplify much of what follows. Moreover, when using finite, rather than infinite, precision it may be more practical to simply cover any arches by a union of Whitney and Carleson boxes.

To construct the covering we fix some $\epsilon>0$, take the hyperbolic convex hull of $S$ and cover it by $O(n) \epsilon$-Carleson arches and Whitney boxes as in Sect. 9. The remaining parts of $Q_{0}$ can be written as a union of Carleson squares $Q$ whose top edges meet the bottom edges of Whitney boxes in the cover above. The part of the boundary of $Q$ that hits the existing cover is a connected piece $E$ of the boundary that contains the top edge. We then subdivide the Carleson box near $E$ into Whitney type and Carleson squares so that any two adjacent squares have comparable size and that subsquares touching $E$ have size comparable to the distance from $\mathbb{R}$; see Fig. 36 to see how to do this. This insures that in our decomposition of $\mathbb{H}$ when two pieces 


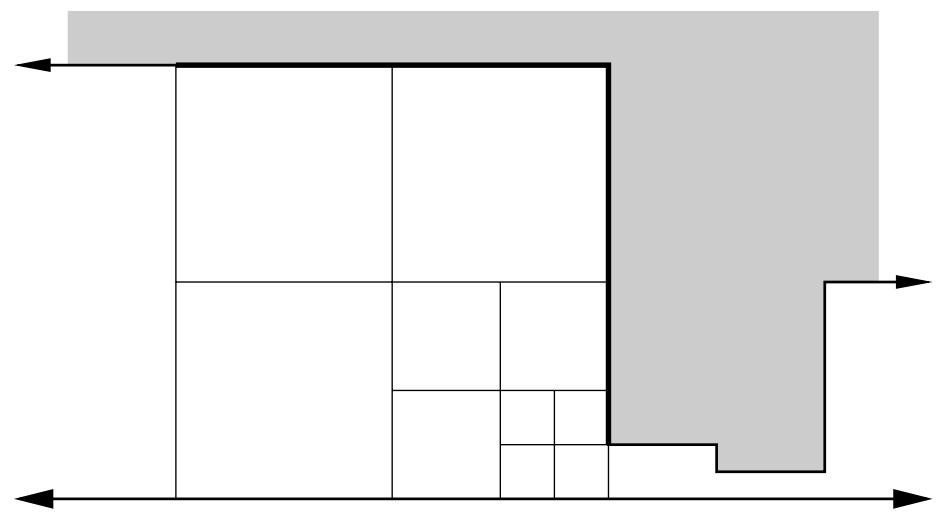

Fig. 36 A Carleson square that lies below our cover of the convex hull can be subdivided into subsquares so that any two adjacent squares have comparable size. The number of squares needed is bounded by a multiple of the number of squares in the covering region and hence is $O(n)$

meet, the intersecting boundary components have comparable size (this is also true for the pieces themselves, except for arches, which may be much larger than adjacent pieces underneath them; however, the lower boundary of the arch is comparable in size to the adjacent pieces). Moreover, the number of squares created is bounded by a multiple of the number of Whitney squares in the cover, so is $O(n)$.

The final step of the construction is to subdivide pieces as follows. Each Whitney square is subdivided into a bounded number of Whitney type squares $Q$ with the property that $M Q$ does not hit a point of $S$ ( $M$ is a fixed large number to be specified later and $M Q$ denotes the box concentric with $Q$ and $M$ times larger); for an example, see Fig. 37. Similarly, we divide Carleson squares into a bounded number of Whitney type squares and Carleson squares so that each can be expanded by a factor of $M$ without hitting $S$ (this can be done since each point of $S$ is contained in a degenerate Carleson arch, and hence any Carleson square in our decomposition is separated from $S$ by a uniform multiple of its diameter). We will call these $M$-Whitney squares or $M$-Carleson squares for $S$. Similarly, we replace arches by thinner arches $Q$ so that $M Q$ misses $S$ and tile the resulting gaps by Whitney type squares and Carleson boxes (for an arch $M Q$ means expanding the bigger square by a factor of $M$ and shrinking the smaller one by a factor of $M$ ).

$M$ will be fixed later to insure various properties. For example, it is easy to verify the following fact we will need using hyperbolic geometry and the fact that Möbius transformations are hyperbolic isometries.

Lemma 19 If $M$ is large enough the following holds. Suppose $Q_{1}$ is an $M$-Whitney square for $S$ and $Q_{2}$ is a $M$-Whitney square for $\tau(S)$ where $\tau$ is a Möbius transformation of $\mathbb{H}$ to itself and that $\tau\left(Q_{1}\right) \cap Q_{2} \neq \emptyset$. Then $2 Q_{2} \subset \tau\left(10 Q_{1}\right)$.

A similar result holds for Carleson squares. For Whitney and Carleson squares,

$$
\operatorname{diam}(Q) \sim \frac{1}{M} \operatorname{dist}(Q, S),
$$



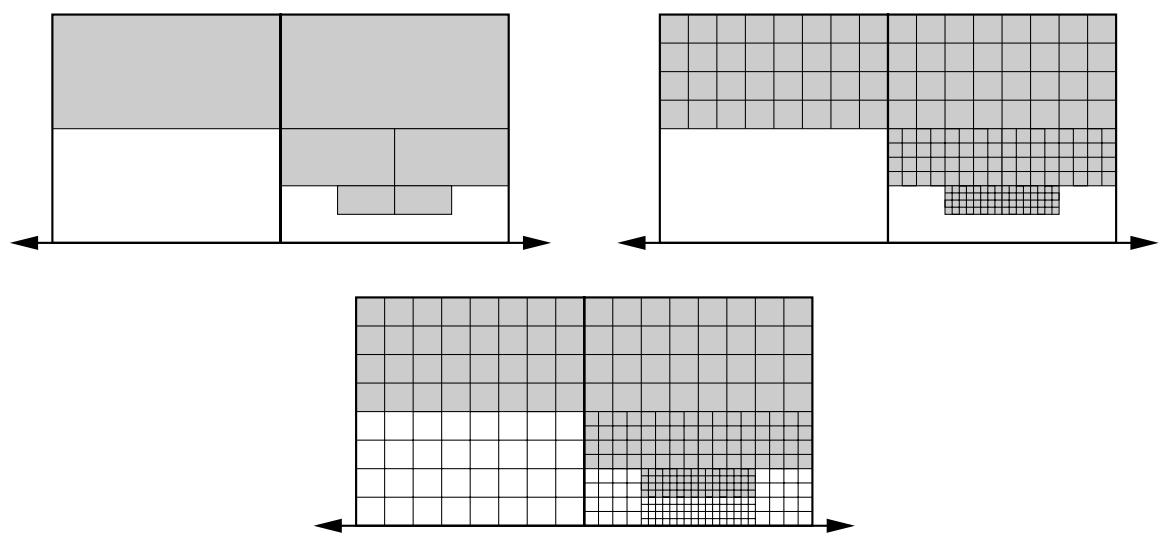

Fig. 37 On the upper left is a collection of Whitney squares, on the upper right a division of these into Whitney type squares and on the bottom we subdivide the region below the Whitney squares into Whitney type squares and Carleson squares so that adjacent squares are of comparable sizes

but this is not true for arches. However, if $E$ is a boundary component of an arch then it is true that

$$
\operatorname{diam}(E) \sim \operatorname{dist}(E, S) .
$$

The pieces of our decomposition form the vertices of a tree, where the parent of a piece is the piece that is adjacent and directly above it. The leaves of the tree are either Carleson squares or degenerate Carleson arches. We will also consider the graph that results from adding edges corresponding to adjacency of pieces (left/right as well as up/down).

Given an element of such a decomposition we consider the part of its boundary in the open upper half-plane. For Whitney type squares, this is a square; for Carleson squares, it is an arc (the top and sides of the rectangle); and for arches, there are two components, each of which is the top and sides of a square. Given a boundary component $E$ of one of these types, we let $\operatorname{diam}(E)$ denote its Euclidean diameter and let $N_{s}(E)$ be the $s \cdot \operatorname{diam}(E)$ Euclidean neighborhood of $E$. Throughout the paper, we only need one fixed value of $s<\frac{1}{4}$, say $s=1 / 10$. Let $N_{s}$ be the union of these sets over all boundary components of all pieces of our decomposition. Similarly, we define $N_{S}(\partial Q)$ to be the union of $N_{S}(E)$ over all boundary components of $Q$ (one such for boxes, two for arches) and let $N_{s}(Q)$ be the union of this with $Q$.

If $Q$ is a piece of our decomposition, we let $z_{Q}$ denote its center (for squares) or the center of the larger square (for arches). Similarly, we let $c_{Q}$ denote its center if $Q$ is a Whitney type square; the center of its base if $Q$ is a Carleson square; and the center of the base of the smaller square if $Q$ is a Carleson arch and the associated point of $S$ for degenerate arches; see Figs. 34 and 38.

Given a disk $D=D(x, s)$ and a number $r>0$ we let $r D=D(x, r s)$. Similarly, given an annulus $A=\{s<|z-x|<t\}$, we let $r A=\left\{s / r<\left|z-c_{Q}\right|<r t\right\}$. With this notation we see that each piece $Q$ of our decomposition contains a disk or half- 

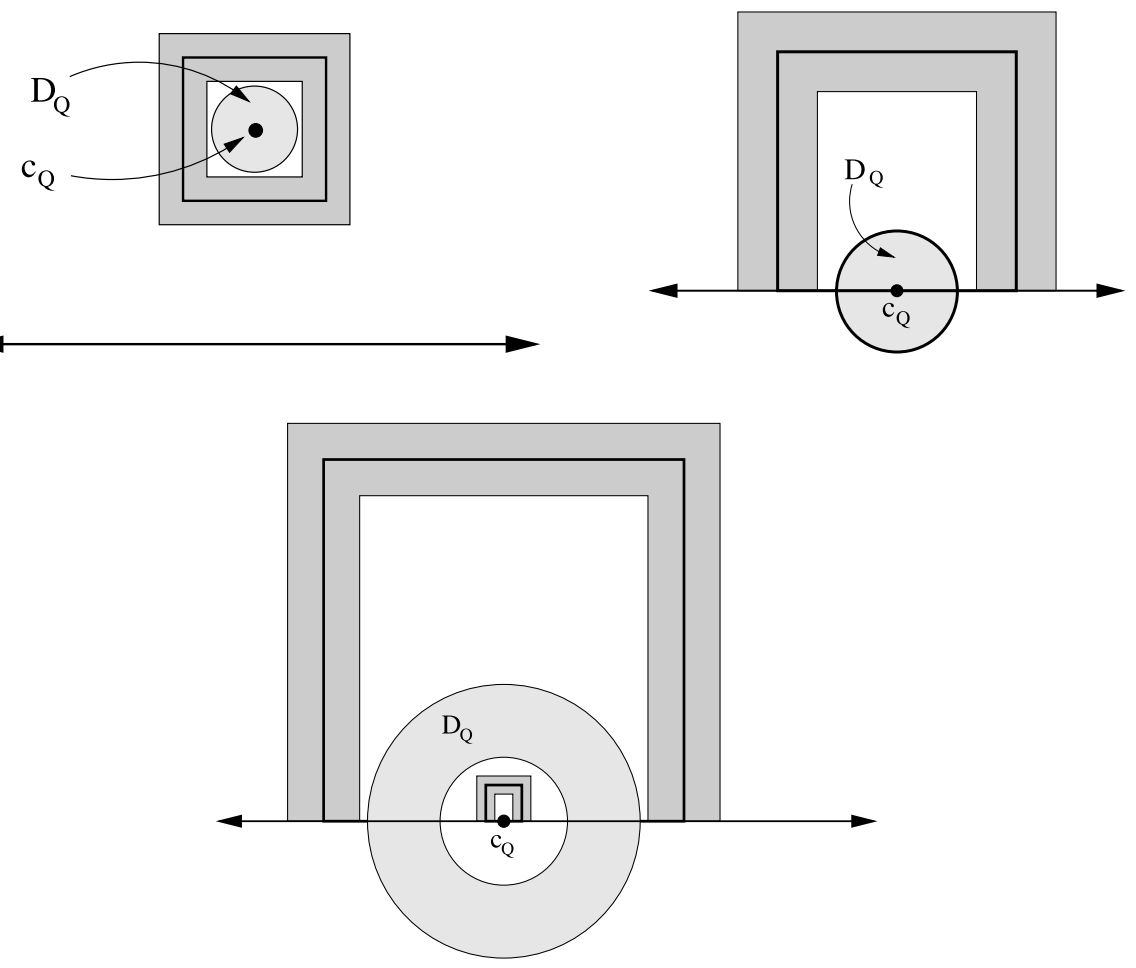

Fig. 38 This shows the boundary neighborhoods (darker), centers and empty regions (lighter) for Whitney squares, Carleson squares and Carleson arches. For a degenerate arch, these are the same as for the Carleson square. The picture of the arch is deceptive since the scales for the two boundary components should be much farther apart, and the empty region much "thicker"

annulus $D_{Q}$ such that

$$
D_{Q} \subset Q \backslash N_{S}(\partial Q) \subset N_{S}(Q) \subset \lambda_{S} D_{Q},
$$

where a little arithmetic shows

$$
\lambda_{s}=\frac{\sqrt{(1+s)^{2}+\left(\frac{1}{2}+s\right)^{2}}}{\frac{1}{2}-s},
$$

and where $D_{Q}$ denotes a disk for square pieces and an annulus for arch pieces; we use the same letter in both cases to simplify notation. We call these the "empty regions" associated to each piece of the decomposition; "empty" because when we define quasiconformal mappings from $\mathbb{H}$ to $\Omega$, the Beltrami dilatation $\mu$ will be supported in $N_{s}$, which is disjoint from $D_{Q}$. Thus our maps will be conformal in the empty regions and so will have power series or Laurent series expansions there. These series will be how we record our quasiconformal maps.

Given the decomposition $\mathcal{W}$ we let $\mathcal{B}$ (for "boundary") be collection of $O(n)$ closed squares with disjoint interiors that lie in $N_{S}$ and whose union cover $N_{S}$; see 


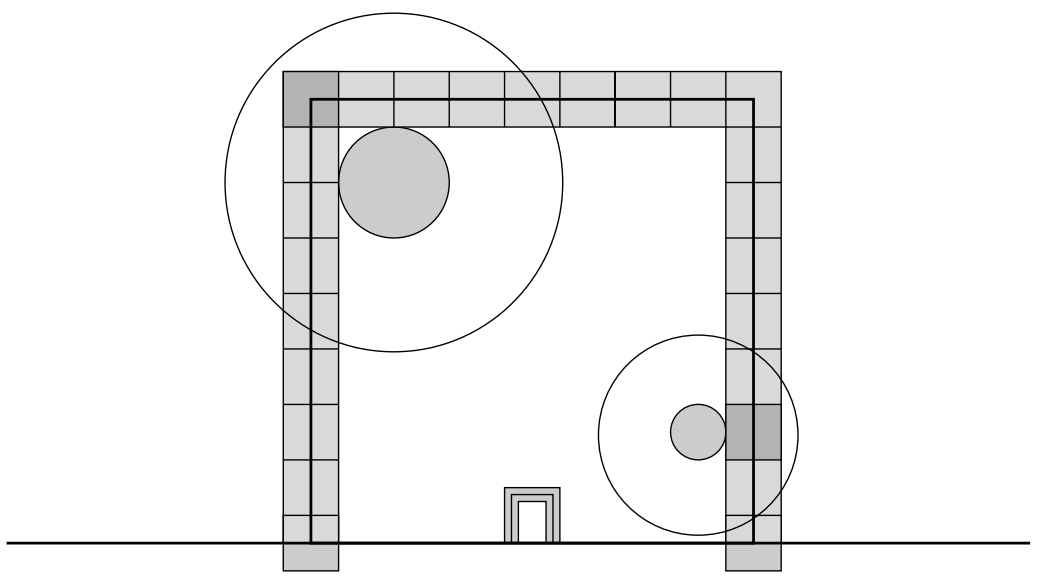

Fig. 39 A neighborhood of an arch boundary can be covered by squares, and each square is associated to a disk inside the arch whose triple covers the square. These disks are the "empty regions" associated to the squares and will be used to define "partial representations" in Sect. 11

Fig. 39. Only a uniformly bounded number of squares are used for each boundary component and each such square is associated to a disk in the arch (and disjoint from the covered neighborhood of the boundary) whose triple covers the square. This disk is the "empty region" associated to the square.

\section{$11 \epsilon$-Representations of Polygonal Domains}

One problem in approximating a conformal map $f: \mathbb{D} \rightarrow \Omega$ (or in the reverse direction) is to decide how to represent the function. One obvious approach would be to use a truncation of the power series of $f, f_{n}(z)=\sum_{k=0}^{n} a_{k} z^{k}$, but this converges slowly (since $f^{\prime}$ is discontinuous at the prevertices) and the number of terms needed for a given accuracy depends on the geometry of the domain. We will use a representation using $O(n)$ different series that avoids these problems.

Given a Carleson-Whitney decomposition $\mathcal{W}$, it is easy to see that there is a corresponding piecewise polynomial partition of unity $\left\{\varphi_{k}\right\}$ whose gradients are supported in $N_{s}$. More precisely, there is a collection of piecewise polynomial functions (uniformly bounded degree) such that

(1) $0 \leq \varphi_{k} \leq 1$,

(2) $\operatorname{supp}\left(\varphi_{k}\right) \subset N_{s}\left(Q_{k}\right)$,

(3) $\sum_{k} \varphi_{k}(z)=1$ for all $z$,

(4) $\operatorname{supp}\left(\nabla \varphi_{k}\right) \subset N_{s}\left(\partial Q_{k}\right)$,

(5) $\left|\nabla \varphi_{k}(z)\right| \leq C /(s \cdot \operatorname{diam}(E))$ for $z \in N_{s}(E)$, when $E$ is a component of $\partial Q_{k}$,

(6) $\left|\nabla^{2} \varphi_{k}(z)\right| \leq C /(s \cdot \operatorname{diam}(E))^{2}$ for $z \in N_{s}(E)$, when $E$ is a component of $\partial Q_{k}$.

To prove this, consider Fig. 40. On the top it shows part of the decomposition and a covering of the boundary arcs by small shaded squares and trapezoids (which allow 

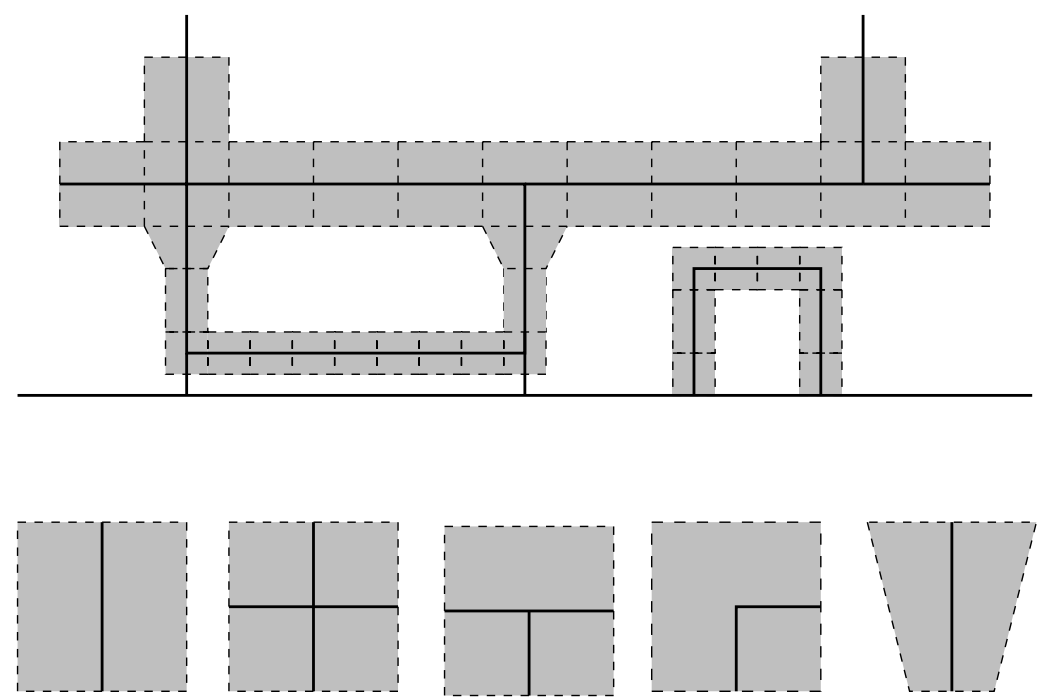

Fig. 40 The partition of unity is non-constant only of these types of regions

the squares to shrink as we approach the boundary). Outside these shaded regions our functions are constant; either 0 or 1 . Within the shaded squares they interpolate between 0 and 1 . There are only five types of regions, and we need only show how to build partition functions for each type.

Let $f(x)=\left(1-x^{2}\right)$ and

$$
g(x)=\int_{-1}^{x} f(t) d t / \int_{-1}^{1} f(t) d t=\frac{1}{2}+\frac{3}{4}\left(x-\frac{1}{3} x^{3}\right) .
$$

Then $g(-1)=0, g(1)=1$ and $g^{\prime}=0$ at these two points (by starting with $f(x)=$ $\left(1-x^{2}\right)^{k}$ we could get higher derivatives to also vanish, if needed). Let $h(x)=g(x)$ if $-1 \leq x \leq 1, h(x)=0$ for $x<-1$ and $h(x)=1$ for $x>1$. For the leftmost square on the bottom of Fig. 40, we can take the functions $\varphi(x, y)=h(2 x)$ and $1-\varphi$ (if $Q=[-1,1]^{2}$; otherwise we apply a Euclidean similarity to make $\varphi$ fit into $Q$ ). These are illustrated in the top row of Fig. 41. The next three squares in Fig. 40 are handled by the functions

$$
\begin{gathered}
h(2 x) h(2 y),(1-h(2 x)) h(2 y),(1-h(2 x))(1-h(2 y)), h(2 x)(1-h(2 y)), \\
h(2 y), h(2 x)(1-h(2 y)),(1-h(2 x))(1-h(2 y)), \\
h(2 x)(1-h(2 y)), 1-h(2 x)(1-h(2 y)),
\end{gathered}
$$

respectively. The trapezoid uses

$$
h(2 x \cdot(2-h(2 y))), 1-h(2 x \cdot(2-h(2 y))) .
$$

It is easy to check that these function have the desired properties. 


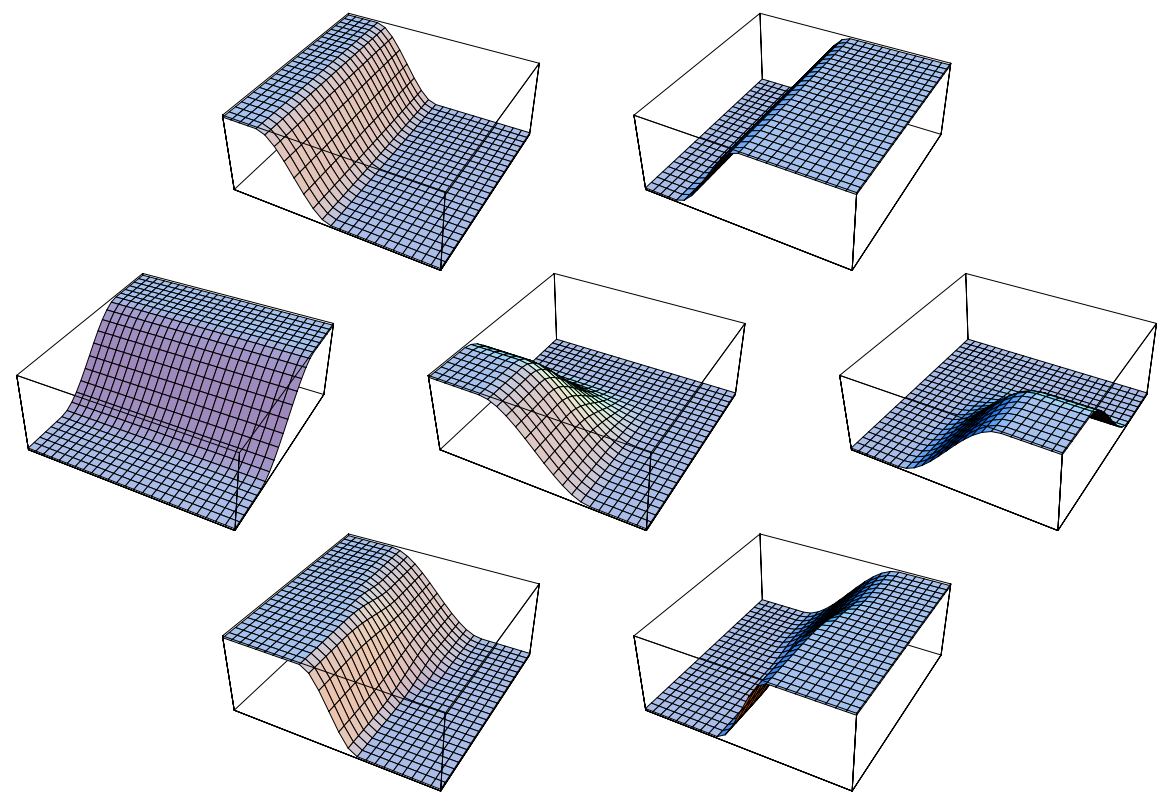

Fig. 41 Some of the functions used in our piecewise polynomial partition of unity. The three rows correspond to the leftmost, center and rightmost regions at the bottom of Fig. 40

Now suppose that for each piece $Q_{k} \in \mathcal{W}$ of our decomposition we have a conformal map $f_{k}$ defined on $N_{s}(Q)$ (we think of this as a local approximation to the globally defined conformal map $f$ ). This collection of functions is denoted $\mathcal{F}$ and has the same index set, $I$, as $\mathcal{W}$. We say that $\mathcal{F}$ represents $\Omega$ if each point of $S$ is mapped to the corresponding vertex of $\partial \Omega$, and each component (if there is more than one) of $\partial Q_{k} \cap \mathbb{R}$ is mapped into the corresponding edge of $\Omega$. Such a $\mathcal{F}$ is an $\epsilon$-representation if each $f_{k}$ is 1-biLipschitz with respect to the hyperbolic metrics and whenever $E_{1}$ and $E_{2}$ are boundary components of pieces such that $N_{s}\left(E_{1}\right)$ and $N_{s}\left(E_{2}\right)$ overlap, then

$$
\left|f_{1}(z)-f_{2}(z)\right| \leq \epsilon \cdot \operatorname{diam}\left(f_{1}\left(E_{1}\right)\right), \quad z \in N_{s}\left(E_{1}\right) \cap N_{s}\left(E_{2}\right) .
$$

We define the norm of our collection, $\|\mathcal{F}\|$ to be the smallest $\epsilon$ for which (3) holds for every pair of adjacent elements of $\mathcal{W}$.

If $E$ is a boundary component of piece $Q_{k}$, define

$$
\partial F(E)=\frac{\operatorname{diam}\left(f_{k}(E)\right)}{\operatorname{diam}(E)} .
$$

If $Q_{k}$ is a Whitney square then $f_{k}$ is conformal on a neighborhood of $Q_{k}$. For other pieces, we can apply Schwarz reflection to see $f_{k}$ can be extended to be conformal on a neighborhood $N_{s}(E)$ for a uniform $s$. So, by Koebe's distortion theorem, $\left|f^{\prime}\right|$ is comparable at any two points of $E$ with uniform bounds. Thus given boundary components $E_{1}, E_{2}$ of two adjacent pieces, the following are all comparable to each 
other and any of them could be used in (3):

$$
\partial F\left(E_{1}\right) \operatorname{diam}\left(E_{1}\right), \quad\left|\partial f_{1}(z)\right| \operatorname{diam}\left(E_{1}\right), \quad\left|\partial f_{2}(z)\right| \operatorname{diam}\left(E_{2}\right), \quad z \in E_{1} \cup E_{2} .
$$

It will be convenient to express (3) in a way that suppresses the $\operatorname{diam}\left(f_{1}\left(E_{1}\right)\right)$ term. Roughly, (3) says that adjacent functions are close to agreeing in the hyperbolic metric of the image domain. This is precisely true for Whitney boxes where $d \rho_{\Omega} \sim d s / \operatorname{dist}(z, \partial \Omega) \sim d s / \operatorname{diam}\left(f_{1}\left(E_{1}\right)\right)$, but not for boundary pieces. We can make it true for all pieces by replacing the hyperbolic metric $\rho_{\Omega}$ by a related metric $\tilde{\rho}_{\Omega}$. Suppose we have a finite set $S \subset \mathbb{T}$ that divides the circle into disjoint $\operatorname{arcs}\left\{I_{j}\right\}$. For each arc, take the disk $D_{j}$ (or possibly a half-plane or disk complement) that intersects $\mathbb{D}$ along this arc and is orthogonal to $\mathbb{T}$. Take the union of these disks and $\mathbb{D}$. The result is a simply connected domain $\Omega_{S}$ that contains $\mathbb{D}$ and so that $S \subset \partial \Omega_{S}$. Let $\tilde{\rho}_{S}$ be the hyperbolic metric on $\Omega_{S}$. If $f: \mathbb{D} \rightarrow \Omega$ is a conformal map to a polygonal domain and $S$ is the set of conformal prevertices, define $\tilde{\rho}_{\Omega}$ on $\Omega$ by pushing $\tilde{\rho}_{S}$ forward by $f$. Clearly, for a boundary component of a decomposition piece in $\mathbb{D}, \operatorname{diam}(E) \sim \operatorname{dist}\left(E, \partial \Omega_{S}\right)$, which means $d \tilde{\rho}_{S} \sim d s / \operatorname{diam}(E)$ on $E$. Therefore, by Koebe's distortion theorem, (3) is equivalent to

$$
\tilde{\rho}_{\Omega}\left(f_{1}(z), f_{2}(z)\right)=O(\epsilon) \text { for all } z \in N_{s}(E) \text {. }
$$

This is a little cleaner looking than (3) and also is more clearly conformally invariant: if $g: \Omega_{1} \rightarrow \Omega_{2}$ is a conformal map of a polygonal domain to a circular arc domain that maps edges into edges then

$$
\tilde{\rho}_{\Omega_{1}}(z, w)=\tilde{\rho}_{\Omega_{2}}(g(z), g(w)) .
$$

In particular, this works for the conformal map to the disk. This will allow us to estimate (4) assuming we have maps into the unit disk; this will be convenient in certain proofs, while the more concrete version (3) is more appropriate for certain explicit calculations. We could also have used the hyperbolic metric on the plane punctured at the points of $E$; away from these points this is approximately the same size as $\tilde{\rho}$, but near each point the asymptotics are different (but we never use the metric there, so either choice would be fine).

Occasionally we will want to measure the distance between a collection $\mathcal{F}$ and a single function $f$ defined on $\mathbb{H}$. We write

$$
\tilde{\rho}_{\Omega}(f, \mathcal{F})=\sup _{j} \sup _{z \in Q_{j}} \tilde{\rho}_{\Omega}\left(f(z), f_{j}(z)\right) .
$$

Given a collection of functions satisfying (3), define

$$
F=\sum_{k \in I} f_{k} \varphi_{k},
$$

Note that whenever we take a non-trivial combination of $f_{k}$ 's along the boundary, the values must lie on the same edge of $\partial \Omega$. Thus any convex combination also lies on this edge, and hence $F$ maps $\mathbb{R}$ to $\partial \Omega$. If we try to define representations of domains 
with curved boundaries, we lose this property (but we shall see how to deal with this in Sect. 13).

Lemma $20 F$ is quasiconformal with constant $1+O(\|\mathcal{F}\|)$, if $\|\mathcal{F}\|$ is small enough.

Proof First, note that $F$ is a continuous mapping of $\mathbb{H}$ into $\Omega$ and that it maps $\mathbb{R}$ onto $\partial \Omega$. If two functions $f_{1}, f_{2}$ are close in the sense of (3), then the Cauchy estimates imply their derivatives are also close in the sense

$$
\left|\partial f_{1}(z)-\partial f_{2}(z)\right| \leq C \epsilon\left|\partial F\left(E_{j}\right)\right|
$$

for $j=1$ or 2 . Note that (all sums are over $k \in I$, the index set of $\mathcal{W}$ ),

$$
\begin{aligned}
& \partial F=\sum_{k}\left(\partial f_{k} \cdot \varphi_{k}+f_{k} \cdot \partial \varphi_{k}\right), \\
& \bar{\partial} F=\sum_{k} f_{k} \cdot \bar{\partial} \varphi_{k},
\end{aligned}
$$

because $\bar{\partial} f_{k}=0$. Since only a bounded number of terms of our partition of unity are non-zero at any point we have

$$
\sum_{k}\left|\nabla \varphi_{k}(z)\right| \leq \frac{C}{\operatorname{diam}(E)}, \quad z \in N_{S}(E) .
$$

Also, since $\sum_{k} \varphi_{k} \equiv 1$, we know $\sum_{k} \partial \varphi_{k}=\sum_{k} \bar{\partial} \varphi_{k}=0$. Note that if $\sum_{k} a_{k}=0$ and $\left|b_{k}-b\right| \leq \epsilon$, then

$$
\left|\sum_{k} a_{k} b_{k}\right|=\left|\sum_{k} a_{k}\left(b_{k}-b\right)\right| \leq \epsilon \sum_{k}\left|a_{k}\right| .
$$

Hence by (3), (7) and (8),

$$
|\bar{\partial} F| \leq \frac{C \epsilon \cdot \operatorname{diam}(f(E))}{\operatorname{diam}(E)} \leq C \epsilon|\partial f(E)| .
$$

Because $\sum \varphi_{k} \equiv 1$,

$$
\partial F=\partial f+\sum\left(\left(f_{k}\right)_{z}-\partial f\right) \varphi_{k}+f_{k} \cdot\left(\varphi_{k}\right)_{z}=\partial f+I+I I .
$$

Thus $|\partial F-\partial f| \leq I+I I$, and we can estimate these terms as

$$
\begin{aligned}
& I \leq \sum_{k} \frac{\left|f_{k}(z)-f(z)\right||\partial F(E)|}{\operatorname{diam}(E)} \leq C \epsilon|\partial f(E)|, \\
& I I \leq \frac{C \epsilon|\operatorname{diam} f(E)|}{\operatorname{diam}(E)} \leq C \epsilon|\partial F(E)| .
\end{aligned}
$$


Thus,

$$
\begin{aligned}
\left|\mu_{F}(z)\right| & =\left|\frac{\bar{\partial} F}{\partial F}(z)\right| \leq\left|\frac{\sum f_{j}(z) \bar{\partial} \varphi_{j}(z)}{\sum \partial f_{j}(z) \cdot \varphi_{j}(z)+f_{j}(z) \cdot \partial \varphi_{j}(z)}\right| \\
& \leq \frac{C \epsilon|\partial F(E)|}{|\partial F(E)|-C \epsilon|\partial F(E)|} \\
& \leq C \epsilon .
\end{aligned}
$$

In particular, if $\|\mathcal{F}\| \leq \epsilon$, then $F$ is a $(1+C \epsilon)$-quasiconformal map of $\mathbb{H}$ to $\Omega$.

Next we define a collection $\mathcal{E}$ of elementary mapping functions. All of these can be considered as conformal maps of the upper half-plane to regions bounded by at most three straight edges (segments, rays or lines) with at most one bounded segment used. As special cases we take the functions $f(z)=a z+b$ (one boundary line), $f(z)=a z^{\alpha}+b$ (two boundary rays) and $f(z)=a \log z+b$ (two parallel boundary lines). When there are three sides there must be two rays and a finite segment and such a map is given by the Schwarz-Christoffel formula; it is elementary in the sense that there is no parameter problem to solve; when there are three sides we can take $\infty$ to map to itself and \pm 1 to map to the finite vertices; see Appendix A.

Suppose $f: \mathbb{H} \rightarrow \Omega$ is conformal and $S$ consists of the preimages of the vertices of $P=\partial \Omega$. The main observation we need to make is that on each piece of our Carleson-Whitney decomposition $Q_{k}$ we can write $f=f_{k} \circ g_{k}$ where $f_{k} \in \mathcal{E}$ and $g_{k}$ is conformal on $M Q_{k}$ for Whitney squares and on $M\left(Q_{k} \cup Q_{k}^{*}\right)\left(Q^{*}\right.$ is the reflection of $Q$ across $\mathbb{R}$ ) for other pieces, and hence $g_{k}$ has a uniformly convergent power or Laurent series on $D_{k}$. In the case of Whitney type squares or Carleson squares with no vertex, this is clear and we can take $f_{k}$ to be the identity. For degenerate arches, this is also clear with $f_{k}$ being a power function, for if the vertex $v$ has interior angle $\alpha$ then $f(z)^{\pi / \alpha}$ can be extended conformally by Schwarz reflection.

The other elements of $\mathcal{E}$ are only needed for arches. In this case, the two components of $\partial M Q \cap \mathbb{R}$ map to line segments, and we can choose $h \in \mathcal{E}$ so that $h^{-1} \circ f$ will map both these to disjoint line segments into $\mathbb{R}$. Hence $h^{-1} \circ f$ will have a conformal extension to $M Q \cup M Q^{*}$. See Fig. 42 .

One of the main difficulties with extending our methods to non-polygons is to define the analogous class of maps $\mathcal{E}$. We shall see in Sect. 13 how to do this for some circular arc polygons, but even in this case problems arise if we need to map two circular arcs into a line simultaneously, so arches can't be used, in general. For more general curved boundaries, it may be difficult even to define maps at the vertices.

Let $g_{k, p}=\sum_{j=-p}^{p} a_{n}\left(z-z_{k}\right)^{j}$ be the truncation of the power or Laurent series for $g_{k}$ around the point $z_{k}=z_{Q_{k}}$. Then since $g_{k}$ is conformal on $10 Q_{k}$, we have

$$
\left|g_{k}(z)-g_{k, p}(z)\right| \leq C \operatorname{diam}\left(g_{k}(E)\right) \lambda^{p},
$$

for a boundary component $E$ of $Q_{k}$ and for $z \in N_{s}(E)$ and some fixed $\lambda<1$. Thus

$$
\left|f(z)-f_{k}\left(g_{k, p}(z)\right)\right| \leq C \operatorname{diam}(f(E)) \lambda^{p},
$$


Fig. 42 This shows parts of a polygon that correspond to using different elements of $\mathcal{E}$. In regions 1 and 2 , we would use the identity, in regions 3 and 4 a power function, a logarithm in region 5 and a 3 -sided Schwarz-Christoffel map in region 6

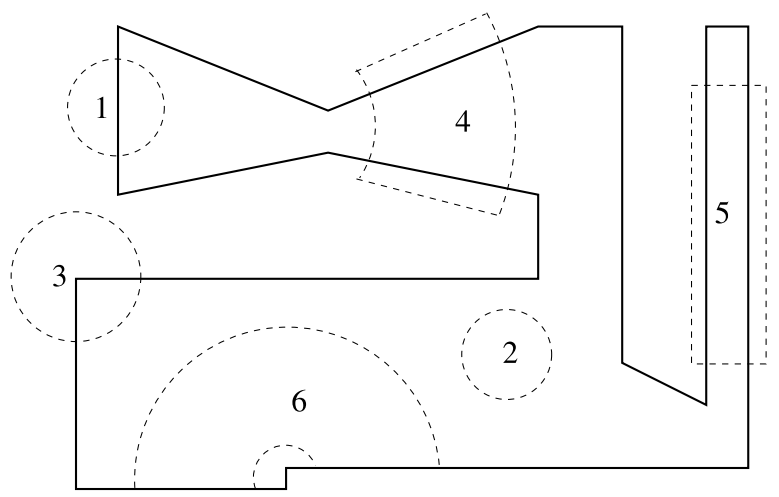

for $z \in N_{S}(E)$ and some fixed $\lambda<1$. We can formalize this a bit with some notation.

An $\epsilon$-representation of $\Omega$ is a triple $(S, \mathcal{W}, \mathcal{F})$ consisting of:

(1) An ordered set $S \subset \mathbb{R}$ of $n$ points.

(2) A Carleson-Whitney decomposition $\mathcal{W}$ of $\mathbb{H}$ with $O(n)$ pieces that extends a covering of the hyperbolic convex hull of $S$, together with the adjacency structure of the pieces and with a piecewise polynomial partition of unity $\left\{\varphi_{k}\right\}$ as described above.

(3) A collection of functions $\mathcal{F}$ of norm $\epsilon$, and each function has the form $f_{k}(z)=$ $h_{k}\left(g_{k}(z)\right)$ where $g_{k}$ consists of $p=O(|\log \epsilon|)$ terms of a power series (for the square pieces) or Laurent series (for the arches) and $h_{k} \in \mathcal{E}$.

It may be a good idea to use Laurent series to implement the algorithm, but we would like to avoid the use of Laurent series in the proof in order to make use of known results about fast manipulations of power series, without having to extend them to allow negative powers. It turns out that our iteration to improve representations only needs the series expansions near the boundary of our decomposition regions and we can always convert a Laurent series to power series on a collection of disks that cover each boundary component. Therefore, we define a partial $\epsilon$-representation as a triple $(S, \mathcal{B}, \mathcal{G})$ where $\mathcal{B}$ is the covering of the boundary of the decomposition introduced at the end of Sect. 10 and $\mathcal{G}$ consists of power series on the corresponding empty regions that satisfy (3). For Whitney or Carleson squares, this is the same as before, but for arches we have "thrown away" the representation in the middle of the arch, keeping power series representations that are valid only near the boundary of the arch.

Clearly, a representation can be turned into a partial representation by restricting an element of $\mathcal{F}$ to a disk and computing its power series there. Conversely, a partial representation can be converted back to a representation by using the power series near the boundary to compute integrals of the form $\int f(z) z^{k} d z$ around the boundary of an arch and thus recover the coefficients of the Laurent series. Moreover, we shall see in Appendix B that these conversions can be done quickly.

Next, we deal with a technical point concerning $\epsilon$-approximations. Later we will want to take power series expansions that are good approximations to the desired 
conformal map on the empty disks of our decomposition and deduce that they are still good approximations on a larger disk that includes the entire piece.

More generally, suppose $f$ is an conformal map defined on $D(0,2)$. Suppose we know the power series of an analytic function $h$, defined on the smaller disk $D(0,1)$ so that $|h-f| \leq \epsilon$ on the smaller disk. Can we use $h$ to get an approximation to $f$ on a larger disk, say $D(0, r)$ for some $1<r<2$ ? Note that $h$ itself may not work. For example, if $f(z)=z$ and $h(z)=z+\left(\frac{8}{9} z\right)^{1000}$ then $h$ is a good approximation for $|z|<1$ but not for $|z|>9 / 8$. However, if we truncate the power series for $h$ appropriately, then we obtain a uniformly good approximation for $f$ on a uniformly larger disk. More precisely:

Lemma 21 There is a $C<\infty$ so that, given $0<\beta<1$ and $1<r=R^{\beta}<R<\infty$, the following holds. Suppose $D=\{z:|z|<t\}$ and suppose $f(z)=\sum_{n=-\infty}^{\infty} a_{n} z^{n}$ is a conformal on $2 R \cdot D=\{z:|z| \leq 2 R t\}$. Suppose also that $h(z)=\sum_{n=0}^{\infty} b_{n} z^{n}$ is holomorphic on $D$ and that $|f-h| \leq \epsilon$ on D. Let $g(z)=\sum_{n=0}^{q} b_{n} z^{n}$ be the truncated power series for $h$ where $q=\left\lfloor c \log \frac{1}{\epsilon}\right\rfloor, c=1 / \log R$. Then

$$
|f(z)-g(z)| \leq \frac{C \epsilon^{1-\beta}}{1-R^{\beta-1}}\left|f^{\prime}(0)\right|,
$$

for all $z \in r D$.

Proof Without loss of generality, we assume $t=1$. Also, normalize $f$ so that $a_{0}=0$ and $\left|a_{1}\right|=1$. Because $f$ is conformal on $2 R D$, this implies $f(R D)$ has uniformly bounded diameter (Koebe distortion theorem, Appendix A). Thus $\left|a_{n}\right| \leq C R^{-n}$, $n \geq 0$ since the usual formula

$$
a_{n}=\frac{1}{2 \pi i} \int_{|z|=R} \frac{f(w) d w}{w^{n+1}},
$$

bounds the coefficients in terms of the maximum of $|f|$. Also, if $\|f-g\|_{\infty}<\epsilon$ for $z \in D$, then $\left|a_{n}-b_{n}\right| \leq C \epsilon$ for $n \geq 0$. The rest of the proof is a simple exercise summing series, i.e., for $1<|z| \leq r$,

$$
\begin{aligned}
\left|f(z)-\sum_{k=0}^{q} b_{k} z^{k}\right| & \leq\left|\sum_{|k|>q+1} a_{k} z^{k}\right|+\sum_{|k| \leq q}\left|a_{k}-b_{k}\right||z|^{k} \\
& \leq C \sum_{k=q+1}^{\infty}\left(\frac{r}{R}\right)^{k}+C \epsilon \sum_{k=0}^{q} r^{k} \\
& \leq \frac{C}{1-(r / R)}\left(\frac{r}{R}\right)^{q}+C \epsilon r^{q} \\
& \leq \frac{C}{1-R^{\beta-1}}\left(\frac{r}{R}\right)^{c|\log \epsilon|}+C \epsilon r^{c|\log \epsilon|} \\
& \leq C \epsilon^{c \log \frac{R}{r}}+C \epsilon^{1-c \log r} .
\end{aligned}
$$


Taking $c=1 / \log R$ and $\beta=\log r / \log R$, the last line becomes $O\left(\epsilon^{1-\beta}\right)$, as desired.

\section{The Thick/Thin Decomposition of a Polygon}

Suppose $\Omega$ is simply connected domain with $n$ specified boundary points $V$, let $f$ be a conformal map of $\mathbb{H}$ onto $\Omega$ and let $S=f^{-1}(V)$. Construct the covering of the hyperbolic convex hull of $S$ as in Sect. 9. If no $\epsilon$-Carleson arches are needed to cover $C(S)$ then we say $(\Omega, V)$ is $\epsilon$-thick (or just "thick", if $\epsilon$ is understood from context). If $\Omega$ has a polygonal boundary we let $V$ be the vertices and this defines a "thick polygon".

A more geometric way to think of thick polygons uses extremal distance. Suppose $e_{1}, e_{2}$ are non-adjacent edges of $\Omega$. We say the pair of edges is $\epsilon$-thin in $\Omega$ if the extremal distance between them is $\leq \epsilon$, i.e., if the modulus of the path family connecting these edges inside $\Omega$ is $\geq \frac{\pi}{\epsilon}$. By our remarks in Lemma 9, such a pair corresponds to an $\delta$-Carleson arch with $\delta \sim \epsilon$. Indeed, thin pairs of non-adjacent sides are in 1-1 correspondence with Carleson arches (except for the possibility that one side has unbounded preimage under $f$, and gives two arches in the decomposition of $\mathbb{H}$ ). Thus if a polygonal domain is $\delta$-thick in the sense of the previous paragraph, then every non-adjacent pair of edges is $\epsilon$-thick for some $\epsilon \simeq \delta$, and conversely. Some examples of thick and not thick polygons are given in Figs. 43 and 44.

We can always add more vertices to the boundary to make a polygon thick, although there is no bound on how many we might have to add. Also note that adding
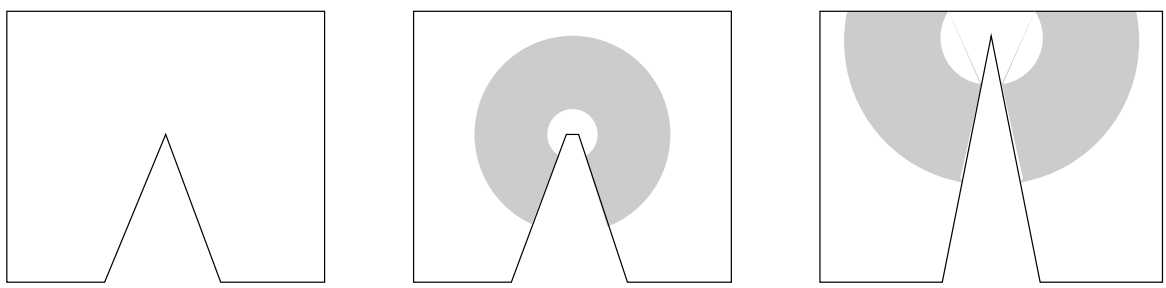

Fig. 43 A thick polygon and two non-thick variations. The shaded areas join non-adjacent edges with small extremal distance and correspond to Carleson arches

Fig. 44 The polygon on top is thick, but not the one on the bottom

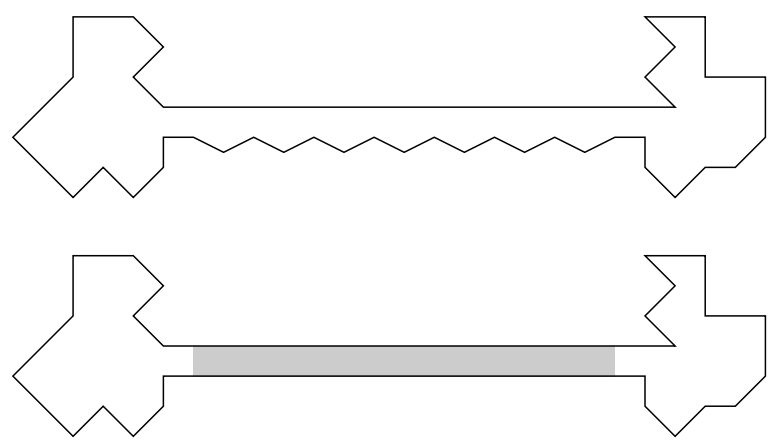


vertices can convert a thick polygon to a non-thick one, i.e., add two vertices $\epsilon$ apart to the middle of an edge of length 1 .

Another way to approach thick and thin parts is to consider the Riemann surface obtained by reflecting the dome of $\Omega$ across $\mathbb{R}^{2}$ and removing the vertices of $\partial \Omega$. This is a punctured Riemann surface (isometric to the unit sphere with the ı-preimages removed) and the thin parts of $\Omega$ correspond to the thin parts of this surface, and the polygon is thick if this surface has no hyperbolic thin parts. We will not pursue this connection between polygons and surfaces further here, but it may prove interesting (e.g., what does Mumford's compactness theorem for surfaces say about polygons?).

Lemma 22 If $(\Omega, V)$ is $\epsilon$-thick then any $K$-quasiconformal image is $\epsilon / K$-thick.

Proof Obvious since conformal modulus can be changed by at most a factor of $K$.

Because of this lemma, we can deduce that a polygon is $\epsilon$-thick by computing the $\iota$ preimages of the vertices and making sure there are no $\epsilon / K$-arches in its covering, where $K$ is the universal quasiconformal bound on the extension of the $\iota$ map to the interior. Thus in time $O(n)$ we can determine (up to a bounded factor of $K$ ) the degree of thickness of a polygon.

It will also be convenient to define a class of polygons called "thin". A thin polygon has two special edges that we call the "long edges", and which may be either adjacent or non-adjacent. In the first case, (which we call parabolic) assume both long edges start at 0 and end at points $\{a, b\} \in A_{R}=\{z: R \leq|z| \leq 2 R\}$. We assume the other edges of the polygon all lie in $A_{R}$, have lengths comparable to $|a-b|$ and interior angles bounded away from 0 and $2 \pi$.

In the second case (which we call hyperbolic), the long edges start at points $a, b$ in $A_{1}=\{z: 1 \leq|z| \leq 2\}$ and end at points $c, d$ in $A_{R}=\{z: R \leq|z| \leq 2 R\}, R \geq 4$. The remaining sides of a thin polygon all lie in either $A_{1}$ or $A_{R}$, have length comparable to $|a-b|$ and $|c-d|$, respectively, and all interior angles are bounded away from 0 and $2 \pi$ (the exact bound is unimportant, but we can take $\pi / 6$ to be specific). We say a polygon is $\epsilon$-thin if there is a Euclidean similarity to shape as described and if the extremal length between the two long sides in the polygon is less than $\epsilon$ and there is no other pair of non-adjacent sides that are $\frac{1}{100}$-thin (again, the exact constant is unimportant). Although the description is a bit wordy, a picture makes it much clearer; see Fig. 45.

The names of the two cases correspond to the thin parts that occur in Riemann surfaces. An $\epsilon$-thin parts of a hyperbolic manifold are the connected components of the set where the injectivity radius of the surface is $<\epsilon$. In the surface case, parabolic thin parts (also called "cusps") are non-compact and have a single (finite) boundary component, whereas hyperbolic thin parts are compact and have two boundary components.

Lemma 23 There is an $\epsilon_{0}>0$ and $0<C<\infty$ so that if $\epsilon<\epsilon_{0}$ then the following holds. Given a simply connected, polygonal domain $\Omega$ we can write $\Omega$ is a union of subdomains $\left\{\Omega_{j}\right\}$ belonging to two families $\mathcal{N}$ and $\mathcal{K}$. The elements of $\mathcal{N}$ are 

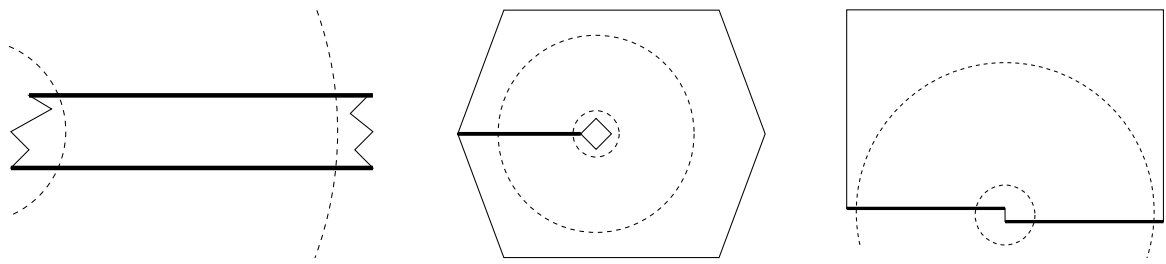

Fig. 45 Examples of hyperbolic thin polygons. The long sides are emphasized and the dashed circles show outer boundary of $A_{1}$ and the inner boundary of $A_{R}$
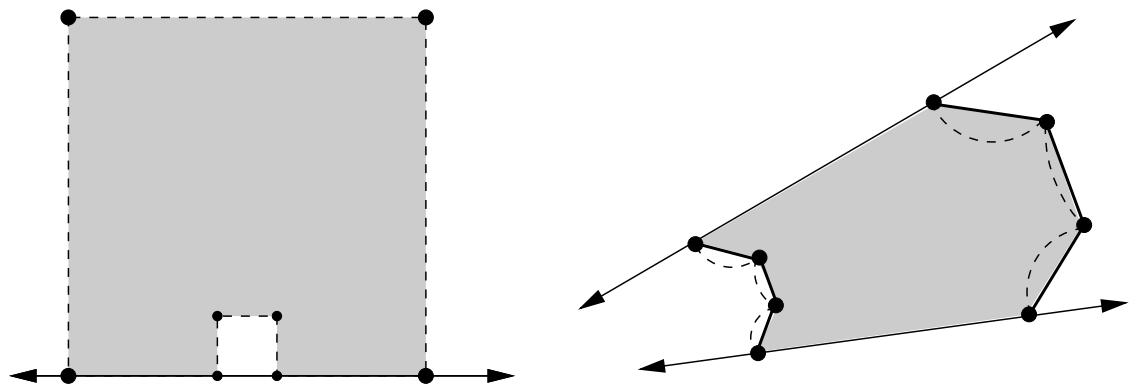

Fig. 46 The intersection of a thin and thick piece is approximately the image of a Carleson arch whose width may be chosen

$O(\epsilon)$-thin polygons and the elements of $\mathcal{K}$ are $\epsilon$-thick. The number of edges in all the pieces put together is $O(n)$ and all the pieces can be computed in time $O(n)$ (constant depends on $\epsilon$ ). We can either choose the pieces to be disjoint except for common boundaries, or given $\epsilon_{0}>\delta>4 \epsilon$, we can choose them so that pieces of each type only intersect pieces of the other type and that the intersection is a $\delta$-thin polygon.

Proof All we have to do is choose polygons that approximate the images of the regions in $\mathcal{N}$ and $\mathcal{K}$ associated to the Carleson-Whitney decomposition in Sect. 10 (see the discussion preceding Lemma 17). Suppose $E$ is a boundary component of a Carleson arch. Then the Euclidean distance of $E$ to the nearest prevertex point is at least $\frac{1}{2} \operatorname{diam}(E)$ and hence we can use Schwarz reflection to show that $f$ extends to be conformal in a ball of radius $\frac{1}{2} \operatorname{diam}(E)$ around every point of $E$. Then the Koebe distortion theorem implies there is a $P<\infty$ so that if we take $P$ equally spaced points along $E$ (including its endpoints on $\mathbb{R}$ ) and connect the $f$ images of these by line segments, the resulting polygonal curve is simple and hence divides $\Omega$ into two polygonal subdomains. See Fig. 46.

When we do this for every domain in $\mathcal{N}$ and $\mathcal{K}$ we get the desired decomposition with the desired overlaps (if the parameter $A$ is chosen correctly). The thin pieces have at most $2 P$ edges. To check that the thick pieces are really thick, we have to show that no two non-adjacent edges have modulus in the piece less than $1 / \epsilon$. If both edges were new ones introduced (e.g., subarcs of a crosscut or a subarc of an original edge created by an endpoint of a crosscut) then this is clear from construction. 
A similar proof works if one edge is new and the other was an original edge. Finally, if both edges are original, then the modulus in the piece is smaller than it is in the whole polygon, and hence the pair is still thick, since it was thick before.

The division of $\Omega$ into thick and thin pieces is not unique because parabolic thin pieces at the vertices either may or may not be included. However, $\mathcal{N}$ must contain all the thin parts corresponding to all the Carleson arches in the decomposition corresponding to $\Omega$. It is easy to estimate the conformal map to thin polygons, at least if we stay near the long edges and away from the other edges.

Lemma 24 Suppose $R$ is the rectangle $[0, L] \times[0,1]$ and $\Omega$ is a simply connected domain containing $R$ that is formed by replacing the two vertical sides of $R$ by curves. Let $f: R \rightarrow \Omega$ be the conformal map such that $f(c)=c$ and $f^{\prime}(c)>0$, where $c=\frac{L}{2}+i \frac{1}{2}$ denotes the center of $R$. Then $|f(z)-z| \leq O\left(e^{-L / 2 \pi}\right)$ for $|z-c|<2$.

Proof Suppose $g: \mathbb{D} \rightarrow R$ and $h: \mathbb{D} \rightarrow \Omega$ are conformal, taking 0 to $c$ with positive derivative at $c$. Then $F=h^{-1} \circ g$ is a conformal map from the disk to $W=h^{-1}(R)$ that fixes the origin and has positive derivative there. Since the extremal length of the path family connecting $B(c, 2) \cap R$ to $\partial \Omega \backslash \partial R$ in $\Omega$ is $\geq \frac{1}{2} L+O(1)$, we get that $\partial W \backslash \mathbb{T}$ has two components, each of Euclidean diameter $\leq \delta$ where $\delta=O\left(e^{-L / 2 \pi}\right)$ by Lemma 43; see Fig. 47. Thus $|F(z)| \geq 1-O\left(e^{-L / 2}\right)$. So if $\log \frac{F(z)}{z}=u(z)+$ $i v(z)$, then $-\delta \leq u \leq 0$ on $\mathbb{D}$ where $\delta=O(\exp (-L / 2))$. By reflection, $u$ extends to be harmonic with $|u| \leq \delta$ on $\mathbb{R}^{2} \backslash E$ where $E=h^{-1}(\partial \Omega \backslash \partial R) \subset \mathbb{T}$ has two components. Thus

$$
|\nabla v|=|\nabla u|=O(\delta / \operatorname{dist}(z, E)),
$$

which together with $v(0)=0$, implies $|u|+|v|=O(\delta)$ on $\left\{z: \operatorname{dist}(z, E) \geq \frac{1}{10}\right\}$, which further implies $|F(z)-z|=O(\delta)$ on the same set. Back on $R$, this means that $|f(z)-z|=O\left(e^{-L / 2 \pi}\right)$, as desired.

Given an $\epsilon$-thin polygonal domain $\Omega$ with long edges connecting $A_{1}$ and $A_{R}$ as above, let $\Omega^{\prime}=\Omega \cap\{z:|z|<R\}$ if $\Omega$ is parabolic and $\Omega^{\prime}=\Omega \cap\{z: 1<|z|<R\}$ if $\Omega$ is hyperbolic. We denote the "center" of $\Omega$ as those points $z$ so that the extremal distance of $E_{z}=\{w \in \Omega:|w|=|z|\}$ to both $A_{1} \cap \partial \Omega$ and $A_{R} \cap \partial \Omega$ is greater

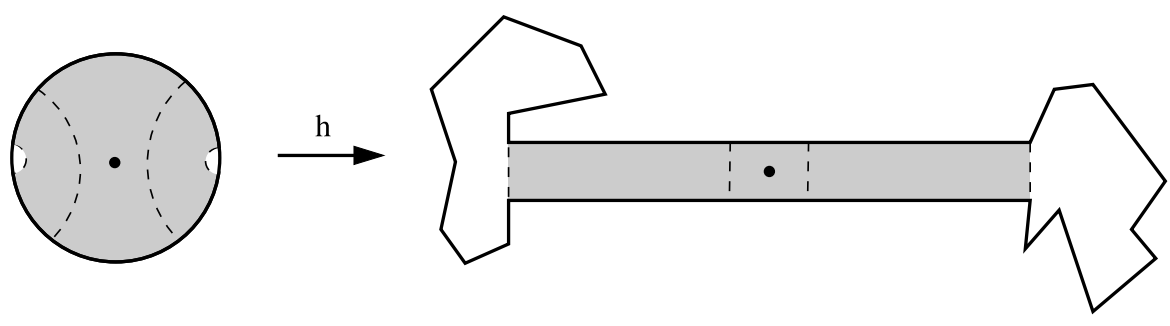

Fig. 47 In the center of a thin polygon, the conformal map is essentially independent of the region outside the center 
than $\epsilon / 2+O(1)$ (in the hyperbolic case) or to just $A_{1} \cap \partial \Omega$ (in the parabolic case). Lemma 24 says that in the center of $\Omega$, the conformal map from $\mathbb{H}$ to $\Omega$ is essentially independent of the part of the boundary outside $\Omega^{\prime}$. In particular, we can chose an elementary mapping from $\mathcal{E}$ (see Sect. 11) that agrees with the conformal mapping onto $\Omega$ up to order $O\left(e^{-2 \pi / \epsilon}\right)$ in the center of $\Omega$.

In Lemma 23, we saw how to break a polygon into thick and thin pieces. Next we observe that we can reconstruct a representation for the whole domain from representations of the thick pieces (since we know maps onto the thin pieces automatically).

Lemma 25 Suppose we are given a decomposition of a $n$-gon into thick and thin pieces (of either parabolic or hyperbolic type) with $\eta$-thin overlaps and we are given a normalized $\epsilon$-representation of each thick piece. Then in time $O(n)$ (constant depending only on $\eta$ and $\epsilon$ ) we can compute a $\delta$-representation of $\Omega$ where $\delta=O\left(\epsilon+e^{-c \eta}\right)$ for some $c>0$.

Proof Choose a thick part $\Omega_{0}$ and think of it as the "root" of a tree where the thick pieces are the vertices and are adjacent iff they are connected by a thin part. In a representation, a thin part corresponds to an arch. This arch has two complementary components; a bounded one and an unbounded one. A normalized representation for each thick part is one where the root lies in the unbounded complementary components of its adjacent hyperbolic thin arches, and the other thick parts lie in the bounded complementary components of the hyperbolic thin part that connects each to its parent.

By choice, the overlap of a thick piece $\Omega_{0}$ and a thin piece $\Omega_{1}$ corresponds to an $\eta$-thin piece. Thus in the decomposition of $\mathbb{H}$ for $\Omega_{0}$, we can find a collection of Whitney boxes and Carleson squares whose union is an $\eta$-Carleson arch. Similarly, if $\Omega_{1}$ is $\epsilon$-thin we have a map of an $\epsilon$-arch to $\Omega_{1}$. Moreover, we can choose a $\eta$-subarch that maps to the same region. We can renormalize the $\epsilon$-arch by linear transformations so that the two $\eta$-arches agree. Then remove the part of the decomposition for $\Omega_{0}$ that lies below the $\eta$-arch corresponding to $\Omega_{1}$ and replace it by an $\epsilon$-arch and the representing function for $\Omega_{1}$; see Fig. 48 .

Now repeat this for the other thin parts adjacent to $\Omega$. If any of these thin parts is adjacent to a second thick part $\Omega_{2}$, then we renormalize the decomposition for $\Omega_{2}$ and insert a copy of the renormalized decomposition for $\Omega_{2}$ below the $\epsilon$-arch of $\Omega_{1}$. Continuing in this way, we eventually insert all the pieces and obtain a $O(\epsilon+$ $e^{-2 \pi / \eta}$ )-representation of the whole polygon.
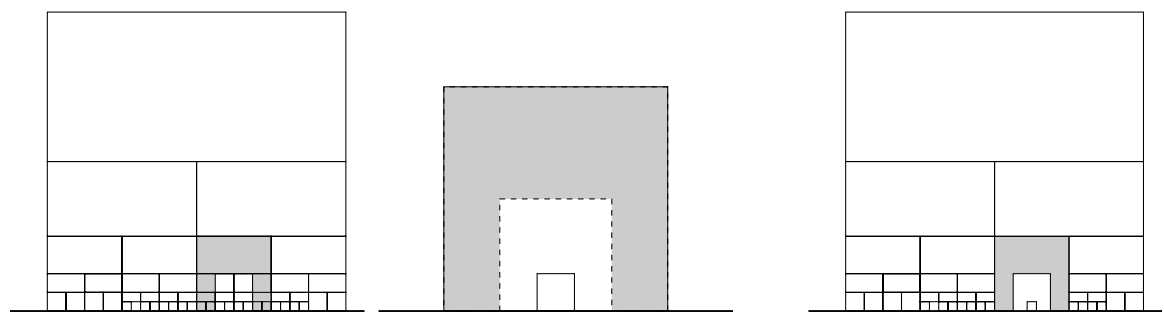

Fig. 48 Combining a representation of a thick piece with an arch from a thin piece 


\section{Representations of Finitely Bent Domains}

One of the main ideas of the algorithm is to connect the given polygonal domain $\Omega$ to the unit disk by a chain of intermediate domains $\Omega_{0}=\mathbb{D}, \Omega_{1}, \ldots, \Omega_{N}=\Omega$ so that each is mapped to the next by an explicit quasiconformal map with small constant. For each domain $\Omega_{k}$, we have to know how to improve a $\epsilon$-representation to any desired accuracy and we have to know how to take a representation for $\Omega_{k}$ and create one for $\Omega_{k+1}$. The improvement step will be discussed in later sections; in this section, we discuss the creation of chain and passing representations from one step to the next.

The conformal maps onto the finitely bent elements on our chain are only computed to a fixed accuracy $\epsilon_{0}$; just enough to allow us to compute the map onto the next element. Thus the precise timing of these steps is not crucial (as long as it is linear in $n$ with a constant depending on $\epsilon_{0}$ ). This gives us an $\epsilon_{0}$-representation of $\Omega$ in time $O(n)$. The time dependence on $\epsilon$ in Theorems 1 and 2 comes from only from iterating Lemma 31 for the domain $\Omega$ in order to improve $\epsilon_{0}$ to $\epsilon$.

If $\Omega$ is finitely bent, we have seen how to create the chain with angle scaling. For a polygonal domain, we will approximate by a finitely bent domain and then use angle scaling on this. At this point we have a choice. One possibility is to replace the chain of finitely bent domains by a chain of inscribed polygons and describe how to pass representations of these polygons from one to the next. The other possibility is to deal directly with the finitely bent chain, but this requires extending the definition of $\epsilon$-representations to such domains (which causes some difficulties as was pointed out earlier). Both approaches involve a similar number of technicalities, but we will take the second one since it is seems cleaner and shows how our method can be adapted to certain domains with curved boundaries.

Suppose $\Omega$ is a finitely bent domain with $n$ vertices. As before, an $\epsilon$-representation is a triple $(S, \mathcal{W}, \mathcal{F})$ where $S$ is a set of $n$ points, $\mathcal{W}$ is the Carleson-Whitney decomposition associated to $S$ and $\mathcal{F}$ consists of a power or Laurent series for each piece and a boundary map for each boundary piece. Each boundary map sends an interval on $\mathbb{R}$ to an subarc of $\partial \Omega$ and each is power function (possibly the identity) followed by a Möbius transformation; see Fig. 49. These maps clearly suffice for Carleson squares and degenerate Carleson arches. However, for such maps to suffice for Carleson arches we have to make an assumption about $\Omega$ : there is a $\delta_{0}>0$, so that whenever two sides of $\partial \Omega$ have extremal distance $<\delta_{0}$ in $\Omega$, the sides lie on intersecting circles. In this case, a power function, followed by a Möbius transformation can be used to map the base intervals of the arch into the sides of $\Omega$. We will call a finitely bent domain with this property simple. It is straightforward to check that this property is preserved by any angle scaling which decreases angles.

Given an $\epsilon$-representation of a simple finitely bent domain, we can define a quasiconformal map $F$ from $\mathbb{H}$ into $\Omega$ using a partition of unity as in Formula (5). However, this map is not onto $\Omega$. The problem is that when we take a convex combination of two points on a circular arc, the result is not on the circle (unless the points coincide). However, if the circle has radius 1 and the points are only $\epsilon$ apart, then it is clear that any convex combination is within $O\left(\epsilon^{2}\right)$ of the boundary. We claim that the domain $F(\mathbb{H}) \subset \Omega$ can be mapped to $\Omega$ by a $1+O\left(\epsilon^{2}\right)$ quasiconformal map whose 
Fig. 49 In region 1, we use a Möbius transform, and in region 2 we can use a power function followed by a Möbius transformation. The same type of function works in the arch 3, since the corresponding circles intersect. However, arches of type 4 are not allowed since it is not clear how to explicitly map the boundary arcs into a single line by a conformal map

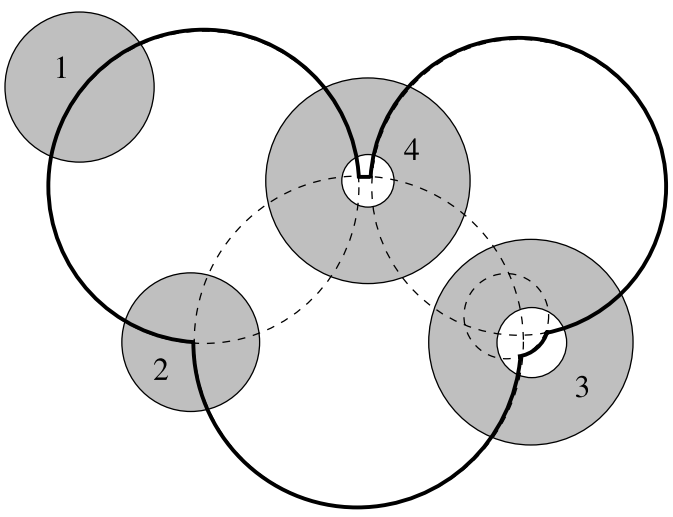

boundary values agree with radial projection on each arc of $\partial \Omega$. However, to prove this, it is not enough to know that the two boundaries are within $O\left(\epsilon^{2}\right)$ of each other; we also need to know that the tangent directions agree to order $O\left(\epsilon^{2}\right)$.

Lemmas 47 and 48 in Appendix A give precise estimates bounding the quasiconformal "cost" of pushing the boundary in this way. We will use the latter to show that $F(\mathbb{H})$ can be mapped to $\Omega$ by a $\left(1+O\left(\epsilon^{2}\right)\right)$-quasiconformal map. After rescaling by linear maps, we may assume the base interval of the boundary piece is $[0,1]$ and the image arc lies on the unit circle. Suppose $x \rightarrow(u, v)$ and $x \rightarrow(a, b)$ are two maps sending $[0,1]$ to an arc of the unit circle with $|u-a|,|v-b|,\left|u^{\prime}-a^{\prime}\right|,\left|v^{\prime}-b^{\prime}\right|=$ $O(\epsilon)$ and suppose $\varphi$ is a partition of unity. Write $F=(\varphi)(u+i v)+(1-\varphi)(a+i b)$ and $g(z)=|F|^{2}=F \cdot \bar{F}=|\varphi(u+i v)+(1-\varphi)(a+i b)|^{2}$. So to apply Lemma 48 to $F$ it is enough to estimate the derivative of $g$. We begin by rewriting $g$ as follows:

$$
\begin{aligned}
g(x) & =(u \varphi+a(1-\varphi))^{2}+(v \varphi+b(1-\varphi))^{2} \\
& =((u-a) \varphi+a)^{2}+((v-b) \varphi+b)^{2} \\
& =a^{2}+b^{2}+2(u-a) a \varphi+(u-a)^{2} \varphi^{2}+2(v-b) b \varphi+(v-b)^{2} \varphi^{2} \\
& =1+2(u-a) a \varphi+(u-a)^{2} \varphi^{2}+2(v-b) b \varphi+(v-b)^{2} \varphi^{2} \\
& =1+2\left(u a-a^{2}+v b-b^{2}\right) \varphi+(u-a)^{2} \varphi^{2}+(v-b)^{2} \varphi^{2} \\
& =1+2((u, v) \cdot(a, b)-1) \varphi+(u-a)^{2} \varphi^{2}+(v-b)^{2} \varphi^{2} \\
& =1+2(\cos \theta-1) \varphi+(u-a)^{2} \varphi^{2}+(v-b)^{2} \varphi^{2},
\end{aligned}
$$

where $\theta$ is the angle between the vectors $(u, v)$ and $(a, b)$, which by assumption is $O(\epsilon)$, as is its derivative $\theta^{\prime}$ (by the Cauchy estimates). If we differentiate $g$, the constant term drops out. The second term is bounded by

$$
2 \sin \theta \cdot \theta^{\prime} \varphi+O\left(\theta^{2}\right) \varphi^{\prime}=O\left(\epsilon^{2}\right),
$$

and the third term is bounded by

$$
\left[(u-a)^{2} \varphi^{2}\right]^{\prime}=2(u-a)\left(u^{\prime}-a^{\prime}\right) \varphi^{2}+(u-a) 2 \varphi \varphi^{\prime},
$$



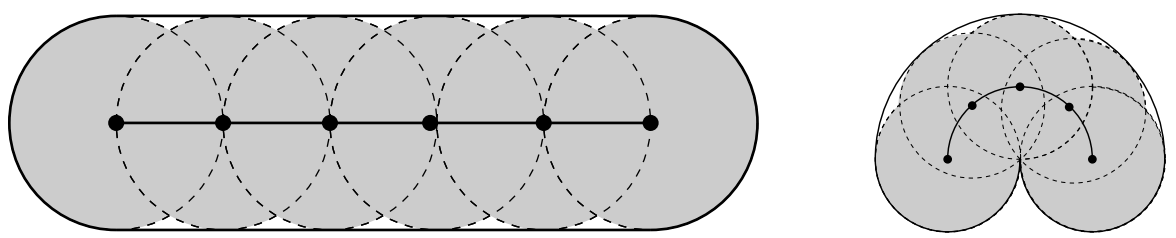

Fig. 50 The finitely bent approximation for a edge-edge bisector and a point-edge bisector (the edge has been mapped to a circular arc)

and each term is $O\left(\epsilon^{2}\right)$. The same estimate holds for the last term. Thus the map $F$ sends $\mathbb{H}$ onto a small quasiconformal perturbation of $\Omega$ (a subset, because of the convexity of disks). Later, in Lemma 31, when we solve the Beltrami equation using the dilatation of $F$ as data, this data differs by $O\left(\epsilon^{2}\right)$ from the dilatation of a quasiconformal map $\tilde{F}$ that sends $\mathbb{H}$ onto $\Omega$. However, the error given by that lemma is also $O\left(\epsilon^{2}\right)$, so using the dilatation of $F$ in place of the dilatation of $\tilde{F}$ gives an error which can be absorbed into this estimate.

Next we wish to approximate a thick polygonal domain $\Omega$ with $n$ sides by a finitely bent domain $\Omega^{\mathrm{fb}} \subset \Omega$ that has $O(n)$ sides. We assume $\Omega$ is thick for otherwise $\Omega^{\mathrm{fb}}$ may require $\gg n$ disks; consider a $1 \times r$ rectangle, $r \gg 1$. The problem is that the medial axis of this polygon has an edge-edge bisector that is very long in the hyperbolic metric. However, if we assume $\Omega$ is $\delta$-thick, then there is an upper bound $A$ on the hyperbolic length of any edge-edge bisector (and $A \simeq \delta^{-1}$ ). This is the only property of thickness that we will use at present.

Fix some $\eta>0$. We say $\Omega^{\mathrm{fb}}$ is an $\eta$-approximation to $\Omega$ if it is the union of the medial axis disks corresponding to all the vertices of the medial axis and also corresponding to certain points chosen along the edges of the medial axis as follows:

Case 1: First, suppose $e$ is a point-point bisector. Then we simply choose the two endpoints of $e$. The union of the two corresponding maximal disks is the same as the union of all the maximal disks corresponding to $e$.

Case 2a: Next suppose $e$ is an edge-edge bisector connecting two vertices of the medial axis in the interior of $\Omega$. Then by the thickness assumption its hyperbolic length is $\leq A$. Thus given $\eta$, we can choose a collection of points on that edge that include the two endpoints and has the property that no point of the edge is more than hyperbolic distance $\eta$ from some point of the collection. We call this being $\eta$-dense. Clearly, we need at most $1+A / \eta$ points per edge; see left side of Fig. 50.

Case $2 b$ : Now suppose $e$ is an edge-edge bisector with one endpoint a convex vertex of $\Omega$. Choose the segment of hyperbolic length $A$ starting at the interior vertex and place points that are $\eta$-dense along it. Clearly, we need at most $1+A / \eta$ points per edge. Note that the finite union of disks has one, $D$, closest to the corresponding convex vertex of $\Omega$. The arc $\partial D \cap \Omega$ closest to the vertex will also be a boundary arc of $\Omega^{\mathrm{fb}}$, and we will call it a "ending arc" of $\Omega^{\mathrm{fb}}$. We place $\eta^{-1}$ equally spaced vertices along the arc to split it into smaller ending arcs. These have the property that the extremal distance to any non-adjacent arc is bounded away from zero (independent of $\eta$ ), a property we will use later.

Case 3: Finally, suppose $e$ is a point-edge bisector. Renormalizing by a Möbius transformation, assume the point is 0 and the edge is a subarc of the unit circle (such 

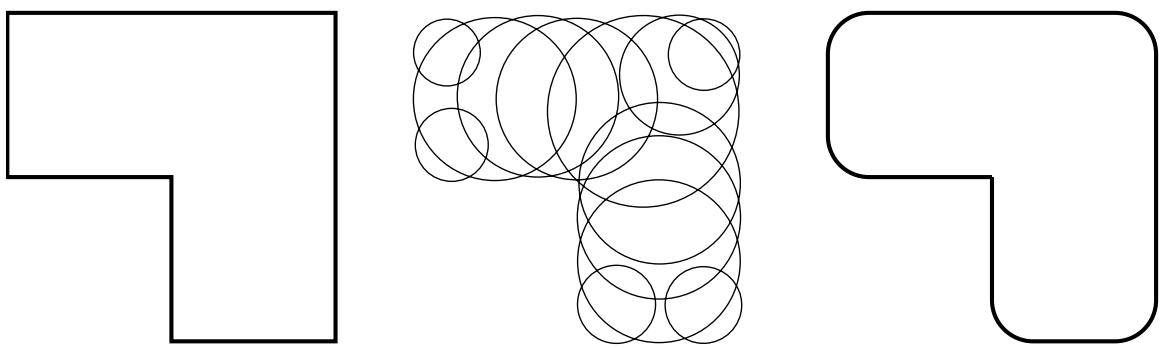

Fig. 51 A polygon $\Omega$, a union of medial axis disks defining a finitely bent approximation $\Omega^{\text {fb }}$ and the corresponding domain with rounded corners, $\Omega^{\text {rc }}$

a transformation preserves the collection of medial axis disks, but does not preserve the medial axis itself since Euclidean centers of a circle need not be preserved by Möbius transformations). In this new region, the medial axis is an arc of the circle of radius $1 / 2$ and we take a collection of points that includes the endpoints, and so that the angular separation between points (when viewed from 0 ) is $\leq \eta$. Clearly, at most $1+2 \pi / \eta$ suffice. Now map this collection of maximal disks back to the original domain to get a collection of disks centered on $e$; see the right side of Fig. 50.

We have shown:

Lemma 26 Suppose $\Omega$ is a $\delta$-thick polygonal domain with $n$ sides and $\Omega^{\mathrm{fb}}$ is an $\eta$-approximation of it. Then $\Omega^{\mathrm{fb}}$ has $O(n /(\delta \eta))$ boundary arcs.

Also note that by construction, each non-ending arc of $\partial \Omega^{\mathrm{fb}}$ is tangent to an edge of $\partial \Omega$, projects orthogonally onto a subsegment of that edge, and makes at most angle $O(\eta)$ with lines parallel to that edge. Using Lemma 47, this means that there is a $(1+O(\eta))$-quasiconformal map from $\Omega^{\mathrm{fb}}$ into a subdomain $\Omega^{\mathrm{rc}}(r c$ is for "rounded corners") of $\Omega$ bounded by the ending edges of $\Omega^{\mathrm{fb}}$ and the projections onto $\partial \Omega$ of the non-ending edges. See Fig. 51.

\section{Really Thick and Almost Thick Pieces}

It would be very convenient if the finitely bent approximation of a thick polygon was also thick, for then the representation of $\Omega^{\mathrm{fb}}$ would not require any arches. This is not the case (see Fig. 52), but there is a substitute that will be sufficient for our purposes. We shall show that if a polygon satisfies a stronger version of thickness, then its finitely bent approximation satisfies a weaker version of thickness. We make this precise with two new definitions.

Suppose $e$ is an edge of a polygonal domain $\Omega$. We call a point $x \in e \subset \partial \Omega$ "exposed" if there is a medial axis disk $D$ with $x \in \partial D$ and so that $\partial D$ also contains a point of an edge that is not adjacent to $e$; see Fig. 53. We say the edge $e$ is exposed if it contains an exposed point in its interior. We will say that $\Omega$ is "really $\delta$-thick" if it is $\delta$-thick and vertices with interior angle $>\pi / 2$ are never endpoints of exposed edges. 
Fig. 52 The polygon is clearly thick, but has interior angle $\pi+\epsilon$ at the vertex $v$. This creates two medial axis vertices whose circles intersect at $w$ which is only $O(\epsilon)$ away from $u$, which must also be a vertex of the finitely bent approximation. Since $\Omega^{\mathrm{fb}}$ has $O(1)$ sides and at least one side shorter than $O(\epsilon)$, it can't be $\delta$-thick if $\epsilon \ll \delta$
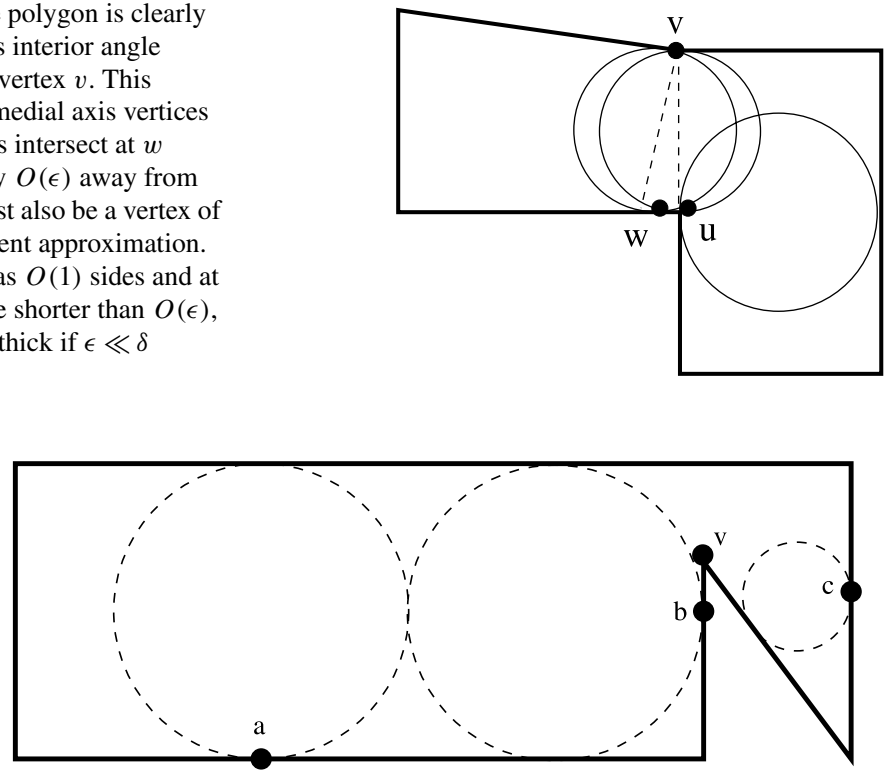

Fig. 53 The points $a, b$ are exposed but the point $c$ is not (nor is the edge containing it). The polygon is thick, but not really thick, because the vertex $v$ has large interior angle and bounds an exposed edge (the one containing $b$ )

Given a $\delta$ thick polygonal domain $\Omega$, we can always modify it to be really $\delta$-thick by adding a bounded number of edges per vertex (thus $O(n)$ overall). Compute the iota map, and the corresponding Carleson-Whitney decomposition. At each vertex with angle $\theta>\pi / 2$, compute the diameter, $d$, of the image of the corresponding degenerate arch, and let $r=\beta d$. The constant $\beta$ is chosen small enough so that $\Omega$ contains a sector of radius $4 r$ and angle $\theta$. On the other hand, $\Omega$ does not contain a sector of angle $\theta$ and radius $>C(\beta) r$, for this would violate the choice of the degenerate arch.

Draw circles of radius $r, 2 r$ around the vertex and replace the part of the edges adjacent to $v$ inside the smaller circle by the polygonal arc as shown in Fig. 54. The new arcs are chosen so that any medial axis disk that hits an interior point has radius $<2 r$, and hence does not hit a third edge of $\partial \Omega$. Thus all these new edges are unexposed. When we finish making this construction at every vertex, the resulting domain is really $\delta$-thick, for all the new edges are unexposed and none of the old adjacent pairs of edges have small extremal distance (if they were separated by a modified vertex).

In particular, given a $\delta$-thick polygonal domain $\Omega$, we can perform a thick/thin decomposition to remove a thin neighborhood of each vertex. Using the construction above we can arrange for the thick component (there is only one since $\Omega$ is thick to being with) to be really thick. If we can construct an $\epsilon$-representation for this really thick domain, then we can use Lemma 25 to obtain a $\delta$-representation of $\Omega$. Thus it suffices to assume that $\Omega$ is really thick. 
Fig. 54 We replace the boundary of $\partial \Omega$ by a polygonal arc near each concave vertex and at a scaled comparable to the largest sector in $\Omega$ around this point. If the original domain is thick, then the new domain is really thick
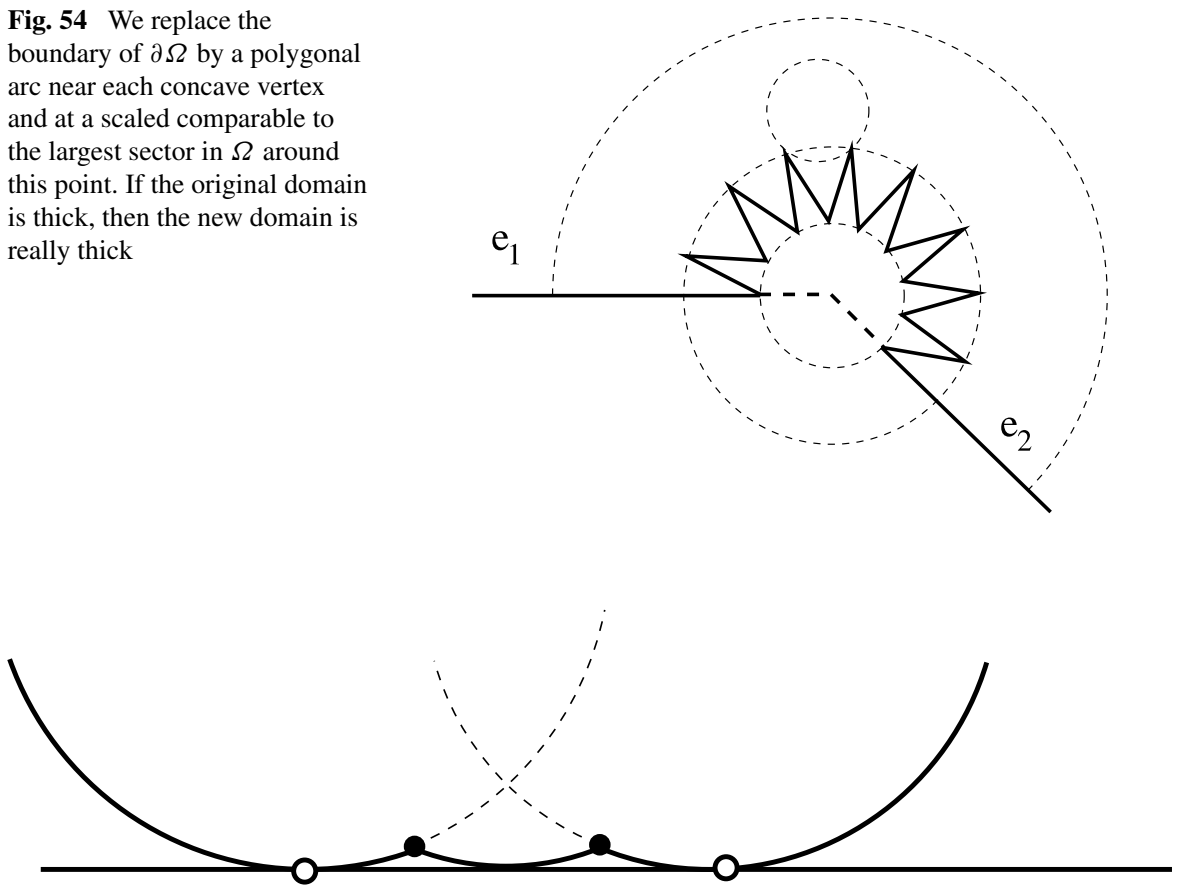

Fig. 55 We have two circular arcs of diameter $\geq 1$, which are distance $\alpha$ apart and both tangent to the same line. Moreover, the endpoints (shown black) are connected by a path of circular arcs of similar radius and tangent to the same line, which implies the distance from the line is $O\left(\alpha^{2}\right)$. Thus the tangent points of the arcs on the line (shown white) are only $O(\alpha)$ apart and the corresponding circles intersect with angle $O(\alpha)$

We say that a finitely bent approximation $\Omega^{\mathrm{fb}}$ of a polygonal domain $\Omega$ is "almost $\delta$-thick" if whenever two non-adjacent $\operatorname{arcs} I_{1}, I_{2}$ have extremal distance $\leq \delta$ in $\Omega^{\mathrm{fb}}$, then these arcs are not ending arcs and they both project onto the same edge of $\Omega$. Thus any arch that occurs in a Carleson-Whitney decomposition corresponding to $\Omega^{\mathrm{fb}}$ has base intervals that map to arcs projecting onto the same edge of $\Omega$.

We defined the normalized separation between $I_{1}$ and $I_{2}$ as

$$
\alpha=\operatorname{dist}\left(I_{1}, I_{2}\right) / \min \left(\operatorname{diam}\left(I_{1}\right), \operatorname{diam}\left(I_{2}\right)\right) .
$$

Since both arcs are tangent to the same line and project orthogonally onto disjoint segments, its easy to see that the circles containing these arcs intersect at angle $O(\alpha)$; see Fig. 55.

The main fact we will use concerns the conformal map $f$ from $D_{1}$ to $D_{1} \cup D_{2}$.

Lemma 27 Suppose $D_{1}, D_{2}$ are disks of comparable size which intersect at angle $\alpha$ and $f: D_{1} \rightarrow D_{1} \cup D_{2}$ is conformal and chosen to fix the two intersection points, $a, b$, and the point $c \in \partial D_{1} \backslash D_{2}$ on the bisector of $a, b$. Then $|f(z)-z|=$ $O(\alpha) \operatorname{diam}\left(D_{1}\right)$ for every $z \in D_{1}$. 

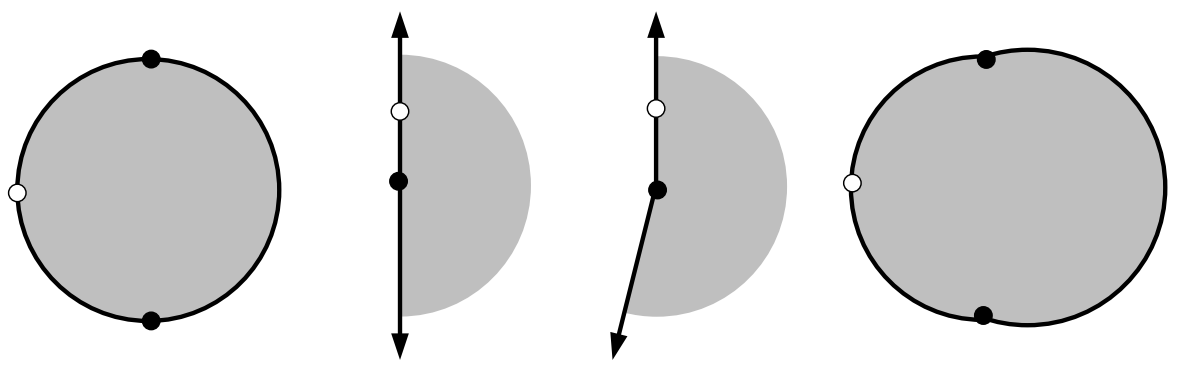

Fig. 56 The disk $D_{1}$ mapped by $\tau, z^{1+\alpha}$ and $\tau^{-1}$. The Möbius transformation $\tau$ sends $a, b, c$ to $0, \infty, i$

Proof This can be proven by an explicit calculation. Note that $f=\tau^{-1}\left(\lambda \tau(z)^{1+\alpha}\right)$ where $\tau(z)=(a-z) /(z-b)$ and $\lambda$ is an appropriately chosen constant of modulus 1 so that $\tau(c)=i$; see Fig. 56. Suppose $z \in D_{1}, w=\tau(z)$ and $x=|w|$. If $0 \leq x \leq 1$, then $x-x^{1+\alpha}$ attains its maximum at its critical points, i.e., at $x=(1+\alpha)^{1 / \alpha}$ which close to $1 / e$ for small $\alpha$. Thus the maximum is $O(\alpha)$ which proves the estimate for such $z$ (because $\tau$ and $\tau^{-1}$ have bounded derivative at such points). The case of $x>1$ is similar using $1 / \tau$.

Lemma 28 If $\delta$ and $\eta$ are small enough the following holds. If $\Omega$ is a really $\delta$-thick polygon, then the finitely bent $\eta$-approximation, $\Omega^{\mathrm{fb}}$, is almost $\delta / 2$-thick.

Proof Suppose we have two non-adjacent arcs, $I_{1}, I_{2}$ in the boundary of $\Omega^{\mathrm{fb}}$. By construction, the extremal distance of an ending arc to any non-adjacent arc is bounded away from zero (independent of $\eta$ ). So we may assume neither of these is an ending arc. Thus they are mapped to line segments $J_{1}, J_{2} \subset \partial \Omega$, under the $(1+O(\eta))$-quasiconformal map $\Omega^{\mathrm{fb}} \rightarrow \Omega^{\mathrm{rc}}$ discussed above. Since $K$-quasiconformal maps change extremal length by a factor of at most $K$, the extremal distance between $J_{1}$ and $J_{2}$ is $\geq(1+O(\eta))(\delta / 2) \leq \delta$ if $\eta$ is small enough. Hence the extremal distance between the edges of $\Omega$ containing them is also $\leq \delta$. Since $\Omega$ is $\delta$-thick, the edges containing $J_{1}$ and $J_{2}$ must be the same or adjacent. If they are adjacent edges, then the common endpoint $v$ must be $\leq \pi / 2$ by the really thick condition. But the arc $I_{1}$ has a tangent point within distance $\alpha \cdot \operatorname{diam}\left(I_{1}\right)$ of the common vertex for a small $\alpha$ (depending on $\eta$ ), which means the circle containing $I_{1}$ can't bound a disk in $\Omega$ (it would hit the other edge hitting $v$ ). This contradiction means $I_{1}, I_{2}$ project onto the same edge of $\partial \Omega$, as desired.

Lemma 29 For any $\epsilon>0$ there is an $N<\infty$ so that the following holds. Suppose $\left\{\Omega_{k}\right\}_{0}^{N}$ is the angle scaling family constructed from an almost $\delta$-thick finitely bent domain $\Omega^{\mathrm{fb}}=\Omega_{N}$ and suppose $0 \leq k<N$. Then given an $\epsilon$-representation of $\Omega_{k}$, we can construct an $2 \epsilon$-representation of $\Omega_{k+1}$ in time $O(n)$ (constant depends on $\epsilon, N)$.

Proof The representation of $\Omega_{k+1}$ uses the same set $S \subset \mathbb{R}$ and the same CarlesonWhitney decomposition $\mathcal{W}$ as is given for $\Omega_{k}$; only the functions will change by 
composing with a conformal map that sends the image from $\Omega_{k}$ to $\Omega_{k+1}$. (This simplicity is why we have gone through the effort of defining almost thick domains; if we allowed more general arches, we would have to update $S$ and $\mathcal{W}$ as well.)

For each Whitney box $Q$, let $f$ be the map defined on $Q$ and choose a gap $G$ in the gap/crescent decomposition of $\Omega_{k}$ which hits $f(Q)$ (or so that an adjacent crescent hits $f(Q))$. This gap is the image under Möbius transformation $\sigma_{k}$, of an ideal triangle in the complement of the bending lamination. The corresponding gap in $\Omega_{k+1}$ is the image of the same triangle under a transformation $\sigma_{k+1}$. We modify the function $f$ associated to $Q$ by post composing by $\sigma_{k+1} \circ \sigma_{k}^{-1}$.

In the previous paragraph, we claimed that for each box $Q$ we could find a gap or crescent that intersects $f(Q)$. We should verify that we can do this for all $O(n)$ boxes in time $O(n)$. This is not immediately clear since arbitrarily many gaps and crescents may hit a particular $f(Q)$, so there is not a constant amount of work to do per box. However, both the Whitney boxes and the gap/crescent decomposition come with tree structures. In time $O(n)$, we can search both trees to find some box and some gap/crescent that intersect. Treating these as roots of their respective trees, we can then search outwards finding the desired intersecting pieces and visiting each vertex of each tree only once (the process is analogous to merging two sorted lists in linear time). The details are given at the end of Appendix A following Lemma 51.

For each of the three kinds of boundary pieces, $Q$, we associate conformal maps $\tau_{k}, \tau_{k+1}$ onto corresponding part of $\Omega_{k}, \Omega_{k+1}$ and form the updated map by composing with $\sigma_{k}=\tau_{k+1} \circ \tau_{k}^{-1}$. We just have to specify the maps $\tau_{k}$ for each type of piece.

If $Q$ is a Carleson square, then it hits a boundary crescent and $\tau_{k}$ is the associated Möbius transformation. For a degenerate arch, the base is an interval containing a point $v$ of $S$ which divides it into two subintervals whose images lie on intersecting circles. We take the map $\tau_{k}$ to be the composition of a power function (determined by the angle of intersection of the circles) and Möbius transformation chosen send $v, \infty$ to the two intersection points of the circles. For an arch $Q$, the base consists of two intervals which are mapped onto two arcs in boundary of the corresponding domain. By construction, $\Omega^{\mathrm{fb}}$ is almost thick, so these two arcs lie on circles which intersect at angle $O(\eta)$ where $\eta$ is the normalized separation between them. The map $\tau_{k}$ is a Möbius transformation of the upper half-plane to $D_{1}$, followed by the conformal map given in Lemma 27.

To estimate the norm of the new collection, we use Lemma 11 (for Whitney boxes and Carleson squares), Lemma 10 (for degenerate arches) and Lemma 27 (for arches). Each result says that the maps $\tau_{k+1} \circ \tau_{k}^{-1}$ are close to the angle scaling map $\psi_{k}: \Omega_{k} \rightarrow \Omega_{k+1}$ which is a quasi-isometry with constants as close to 0 as we wish by taking $N$ large. Thus for any point in overlapping neighborhoods of adjacent pieces $Q_{i}, Q_{j}$,

$$
\begin{aligned}
\left|f_{i}^{k+1}(z)-f_{j}^{k+1}(z)\right| \leq & \left|f_{i}^{k+1}(z)-\psi_{k}\left(f_{i} k(z)\right)\right| \\
& +\left|\psi_{k}\left(f_{i}^{k}(z)\right)-\psi_{k}\left(f_{j}^{k}(z)\right)\right|\left|\psi_{k}\left(f_{j}^{k}(z)\right)-f_{j}^{k+1}(z)\right| .
\end{aligned}
$$

The middle term is small if $\mathcal{F}=\left\{f^{k}\right\}$ has small norm and $\psi_{k}$ has quasi-isometry constant close to 0 . The first and last terms are small since $\sigma_{j}^{k}$ is close to $\psi_{k}$. 
Lemma 30 Given an $\epsilon$-representation of $\Omega^{\mathrm{fb}}$ (the finitely bent $\eta$-approximation to a really thick polygonal domain $\Omega$ ) we can construct an $2 \epsilon$-representation of $\Omega$ in time $O(n)$, assuming $\eta$ is chosen small enough (depending on $\epsilon$, but not on $n$ or the geometry of $\Omega$ ).

Proof The proof is similar to the previous proof. As above, we leave the set $S$ and the decomposition the same, and only change the maps. For Whitney boxes, we compose with the identity (i.e., use the same map). We do the same for boundary pieces corresponding to ending arcs. For Carleson squares corresponding to non-ending arcs, we compose by the Möbius transformations that maps the boundary arc onto its orthogonal projection on $\partial \Omega$ (with the tangent point going to itself). For degenerate arches, we use the power function composed with Möbius transformation that sends the boundary arc into its orthogonal projection with the vertex going to its projection. For arches we use the conformal map of $D_{1} \cup D_{2}$ onto the half-plane bounded by the line containing the corresponding boundary edge. Since the diameter of the boundary pieces $W$ are comparable to the lengths of the boundary arcs they hit, each type of maps moves points by less than $O(\delta) \operatorname{diam}(W)$. Thus an $\epsilon$-representation is converted to a $(\epsilon+O(\delta))$-representation by taking $\delta$ small enough.

So given any polygonal domain, we perform a thick/thin decomposition to remove the hyperbolic thin parts. For each resulting thick part, we decompose it into a really thick part and its parabolic thin parts. We then take a finitely bent approximation of each really thick components, and using angle scaling chains to inductively construct representations of the really thick components. We then combine these with the explicit representations of the thin components to get a representation of the original domain using Lemma 25. This completes proof of Theorems 1 and 2 except for the proof of Lemma 31.

\section{Iterating to the Solution}

In this section, we will show how to improve a partial $\epsilon$-representation quickly assuming a certain $\bar{\partial}$-problem can be solved quickly. The following is our Newton-type iteration for improving an $\epsilon$-representation. The fact that $\epsilon_{0}$ does not depend on $\Omega$ is one of the pillars upon which the whole proof rests.

Lemma 31 There is an $\epsilon_{0}>0$ so that if $0<\epsilon<\epsilon_{0}$ then the following holds. Given a partial $\epsilon$-representation of a polygonal region $\Omega$ we can construct a partial $\epsilon^{2}$ representation in time $O\left(n \log \frac{1}{\epsilon} \log \log \frac{1}{\epsilon}\right)$.

Recall the notation $\bar{\partial} f=f_{\bar{z}}=\frac{1}{2}\left(f_{x}+i f_{y}\right), \partial f=f_{z}=\frac{1}{2}\left(f_{x}-i f_{y}\right)$. The Beltrami dilatation of a map is given by $\mu_{f}=f_{\bar{z}} / f_{z}=\bar{\partial} f / \partial f$.

Suppose $\left(S_{0}, \mathcal{B}_{0}, \mathcal{G}_{0}\right)$ is a partial $\epsilon$-representation of the domain (recall this means $S$ is a set of $n$ points giving our current guess of the prevertices, $\mathcal{B}$ is the covering of the corresponding decomposition, and $\mathcal{G}$ is a function defined on each decomposition piece). Let $F$ denote the quasiconformal map associated to this $\epsilon$ approximation 
by (5). Then $\mu_{F}$ is a piecewise rational function bounded by $\epsilon$ and we can estimate it to within $\epsilon^{2}$ by a polynomial on each piece with at most $O(p)$ terms (using the geometric sum formula). More precisely, if $Q_{k}$ is a decomposition piece and $z \in N_{s}\left(Q_{k}\right)$, then

$$
\mu_{F}(z)=\frac{\sum f_{j}(z) \bar{\partial} \varphi_{j}(z)}{\sum \partial f_{j}(z) \cdot \varphi_{j}(z)+f_{j}(z) \cdot \partial \varphi_{j}(z)} .
$$

Let $\mu$ be the symmetrized version (with respect to the real axis) of this approximation, i.e., $\mu$ is extended to the lower half-plane by $\mu(\bar{z})=\overline{\mu(z)}$. Any solution of the Beltrami equation with this data is also symmetric, so maps the real line to itself. Note that $\|\mu\|_{\infty}=O(\epsilon)$ and is supported on the $O(n)$ squares that cover the boundary of our Carleson-Whitney decomposition (and its reflection in the lower half-plane). If $\|\mu\|_{\infty} \leq \epsilon$, we wish to find a quasiconformal map $H$ of $\mathbb{H}$ to itself that satisfies

$$
\mu_{H}=\mu+O\left(\epsilon^{2}\right) .
$$

Then $G=F \circ H^{-1}$ is a quasiconformal map of $\mathbb{H}$ to $\Omega$ with quasiconformal constant $1+O\left(\epsilon^{2}\right)$.

Let $p=O(|\log \epsilon|)$. Suppose we can find a $p$-term series expansion for $H$ in each empty piece of the decomposition. Then we could compose $H^{-1}$ with our existing representation for $F$ to get the $p$ first terms of series approximating $F \circ H^{-1}$ on the empty pieces of the Carleson-Whitney decomposition associated to $H\left(S_{0}\right)$, and this series is accurate to within $\tilde{\rho}_{\Omega}$-distance $O\left(\epsilon^{2}\right)$. We can then apply Lemma 21 to obtain a $O\left(\epsilon^{\alpha}\right)$ representation for some $\alpha>1$. Iterating this a fixed number of times (depending on $\alpha$ and the constant in the " $O$ ") gives an $\epsilon^{2}$-representation.

In the remainder of this section, we show how to define the new representation of $\Omega$, given the expansions for $H$. In the next section we show how to define $H$, assuming we can solve a certain $\bar{\partial}$-problem, and in Sect. 17 we show how to solve this $\bar{\partial}$-problem.

How do we define the new representation for $\Omega$ ? Suppose $\left(S_{0}, \mathcal{B}_{0}, \mathcal{G}_{0}\right)$ is the previous partial $\epsilon$-representation with dilatation $\mu$ and that $H$ is a $(1+O(\epsilon))$ quasiconformal map of $\mathbb{H}$ to itself that solves $\mu_{H}=\mu+O\left(\epsilon^{2}\right)$. Moreover, $H$ is conformal on the empty pieces of our decomposition and we have power series on each empty piece $D$ that agree with $H$ to within $O\left(\epsilon^{2} \operatorname{diam}\left(f_{k}(E)\right)\right)$ on $N_{S}(E)$ where $E$ is a boundary component of $Q_{k}$.

The points $S=H\left(S_{0}\right)$ are known, and we take these to be the first part of our new triple. In time $O(n)$, we can compute a covering of the hyperbolic convex hull of $S$ and extend this to a decomposition of $\mathbb{H}$. This will be our new $\mathcal{B}$. For each Whitney square $Q$ in $\mathcal{B}$, choose a Whitney square $Q_{0} \in \mathcal{B}_{0}$ so that $H\left(Q_{0}\right)$ hits $Q$ (we can do this in bounded time since $H$ is almost an isometry and we need only search a uniformly bounded number of possible squares). Then there is a ball $B$ so that $Q \subset B \subset H\left(5 Q_{0}\right)$ and we can compute the inverse $h_{Q}$ of the power series of $H$ on $B$ in and then compute the composition of that series followed by the series for $Q_{0}$ (which converges on $10 Q$ ). This gives the element of $\mathcal{F}$ corresponding to $Q$. This computation takes time $O(p \log p), p=\log \frac{1}{\epsilon}$ (see Lemma 52 of Appendix B). A truncation of this series, as given by Lemma 21 , will give the desired element of $\mathcal{F}$. To see how, we use the following result. 
Lemma 32 Suppose $f: \mathbb{D} \rightarrow \mathbb{D}$ is $\left(1+\epsilon^{2}\right)$-quasiconformal and is conformal on the disk $D(0,1 / M)$ and maps 0 to 0 . Then there is a truncation $g$ of the power series of $f$ at 0 , such that $|g(z)-f(z)| \leq O\left(\epsilon^{2(1-\beta)}\right)$ on $D\left(0, M^{\beta-1}\right)$.

Proof Since $f$ is $\left(1+\epsilon^{2}\right)$-quasiconformal and fixes the origin, it agrees with a rotation $\tau$ to within $O(\epsilon)$. Apply Lemma 21 to deduce the result.

Our previous remarks prove:

Lemma 33 If $\|\mu\|_{\infty}=\epsilon<\epsilon_{0}$ then $\|\mathcal{G}\| \leq O\left(\epsilon^{2(1-\beta)}\right)$.

Thus if $\beta<1 / 2$ and $\epsilon_{0}$ is small enough we get a definite improvement, and a bounded number of iterations will improve it below $\epsilon^{2}$. This choice of $\beta$ determines the choice of $M$ in the construction of the decomposition in Sect. 10.

\section{Reducing the Beltrami Problem to a $\bar{\partial}$ Problem}

In Appendix A, we recall that the Beltrami equation $\bar{\partial} f=\mu \partial f$ can be solved using an infinite series of the form $T \mu+T \mu T \mu+\cdots$, where $T$ is the Beurling transform. The method we describe below might be adapted to use several terms of this series, but we shall use only the leading term.

We will replace the Beltrami equation $\bar{\partial} H=\mu \partial H$ with the easier equation $\bar{\partial} H=\mu$. For $\epsilon$ small, we will show $\partial H$ is close to a constant on each piece of our decomposition, which means the solution of the Beltrami problem is close to a constant multiple of the solution of the $\bar{\partial}$-problem. Since $\mu$ has compact support, the $\bar{\partial}$-problem can be exactly solved by the convolution

$$
G_{1}(z)=\frac{1}{2 \pi i} \iint \frac{\mu(w) d x d y}{z-w} .
$$

It will actually be slightly more convenient to deal with $\partial G_{1}$, which is given by the Beurling transform $T \mu$,

$$
\partial G_{1}(z)=T \mu(w)=\lim _{r \rightarrow 0} \frac{1}{2 \pi i} \iint_{|z-w|>r} \frac{\mu(z)}{(z-w)^{2}} d x d y .
$$

This convolution gives a solution of $\bar{\partial} G_{1}=\mu$, but we cannot compute $\partial G_{1}$ exactly in finite time. However, we can compute $p$ terms of a power or Laurent series that approximates $\partial G_{1}$ in each of the empty pieces of our decomposition (where $G_{1}$ is holomorphic). We will refer to the expansion on a piece $Q$ as $G_{Q}$. We will see later that for each piece $Q$ of our decomposition we can compute an expansion $G_{Q}$ so that

$$
\left|\partial^{2} G_{1}(z)-\partial^{2} G_{Q}(z)\right| \leq \frac{C \epsilon^{2}}{\operatorname{diam}\left(D_{Q}\right)}, \quad z \in D,
$$

where $D$ denotes the empty region of $Q$. (The $\epsilon^{2}$ could be replaced by a higher power of $\epsilon$, if necessary, by simply taking more terms in $G_{Q}$.) 
It will also be convenient to consider a function $G_{2}$ on $\mathbb{H}$ so that (1) $G_{2}=G_{1}$ on $N_{s}$ (recall this is the union of $N_{s}(E)$ over all boundary components of all pieces), (2) $G_{2}=G_{Q}$ on $D_{Q}$ and (3) $\bar{\partial} G_{2}=\bar{\partial} G_{1}+O\left(\epsilon^{2}\right)$. This can easily be obtained by combining $G_{1}$ and the $G_{Q}$ 's using a partition of unity whose gradient is supported in $N_{S}(D) \backslash D$, whose gradient in bounded by $O\left(\operatorname{diam}(E)^{-1}\right)$ and whose second gradient is bounded by $O\left(\operatorname{diam}(E)^{-2}\right)$ on $N_{S}(E)$, when $E$ a boundary component of $D$. Note that this implies

$$
\left|\partial G_{1}(z)-\partial G_{2}(z)\right| \leq C \epsilon^{2} .
$$

The algorithm only requires the computation of $\partial G_{Q}$, not of $\partial G_{2}$; the latter function will only be used in the proof that the algorithm gives the desired accuracy.

Lemma 34 Suppose $E$ is a boundary component of a piece $Q$. Then $\left|\partial^{2} G_{1}(z)\right| \leq$ $C \epsilon / \operatorname{diam}(E), z \in N_{S}(E)$. The same estimate also holds for $G_{2}$.

Proof Assume $z \in N_{s}(E)$. Let $D$ be a Euclidean disk of radius $r \simeq \operatorname{diam}(E)$ around $z$ and let $\chi_{D}$ denote its characteristic function $(=1$ on $D$ and 0 off $D)$. Let $L(w)$ be the linear function such that $|\mu(w)-L(w)| \leq C \epsilon|z-w|^{2} / r^{2}$ for $w \in D$ (there is such a function because we constructed $\mu$ to have second derivative bounded by $\left.C \epsilon / r^{2}\right)$. Then,

$$
\begin{aligned}
&\left|\partial^{2} G_{1}(z)\right| \simeq\left|\iint \frac{\mu(w) d x d y}{(z-w)^{3}}\right| \\
& \lesssim \iint \frac{\left|\mu(w)-L(w) \chi_{D}(w)\right| d x d y}{|z-w|^{3}} \\
&+\left|\iint_{D} \frac{L(w) d x d y}{(z-w)^{3}}\right|+\iint_{D^{c}} \frac{\epsilon d x d y}{|z-w|^{3}} \\
& \lesssim C \epsilon \iint_{D} \frac{d x d y}{r^{2}|z-w|}+0+C \epsilon / r \\
& \leq
\end{aligned}
$$

The final claim holds because $G_{1}=G_{2}$ on $N_{s}(E)$.

Corollary 35 Suppose $Q$ is a piece of our decomposition. Then $\mid \partial G_{2}(z)-$ $\partial G_{2}\left(z_{Q}\right) \mid \leq C \epsilon$ for every $z \in Q$.

Proof Recall the definition of $z_{Q}$ (see Fig. 34). For Whitney type squares and Carleson squares, there is only one boundary component and the proof of Lemma 34 actually applies to any point in $Q$ and then we integrate along a segment connecting $z$ and $z_{Q}$ and note that the length of the segment at most $O(\operatorname{diam}(E))$.

For arches, it is slightly more involved since there is a boundary component that is much smaller than the diameter of the whole piece. Since $\partial G_{2}$ is holomorphic in the empty part of the piece, the maximum principle implies it is enough to check the inequality near the boundary components. The proof for the "big" boundary component 
is just like the case of Whitney squares and Carleson boxes. For the 'small' boundary component, it suffices to check the estimate at one point near that component, say the one directly underneath $z_{Q}$ and distance $2 s \cdot \operatorname{diam}(E)$ above $E$. Then

$$
\partial^{2} G_{1}=O\left(\iint \frac{|\mu(w)|}{|z-w|^{3}} d x d y\right),
$$

and we can break the integral into two parts corresponding to the two complementary components of the annulus. The unbounded part is bounded by $O\left(\epsilon \operatorname{diam}(Q)^{-1}\right)$ and the other is bounded by $O\left(\epsilon t^{-3} \operatorname{diam}(E)^{2}\right)$ for a point at height $t$. Integrating both estimates from $t=\operatorname{diam}(E)$ to $t=\operatorname{diam}(Q)$, gives $O(\epsilon)+O(\epsilon)$ and is an upper bound for the variation of $\partial G_{1}$ along the vertical line segment connecting the two boundary components. This proves the desired estimate for $G_{1}$. It follows for $G_{2}$ by (11).

Given the function $G_{2}$, which is an approximate solution of the $\bar{\partial}$-problem, we can define a quasiconformal mapping on each piece of our decomposition. Assume for the moment that $z_{Q}$ maps to $w_{Q}$ and let $d_{Q}=\operatorname{Im}\left(w_{Q}\right) / \operatorname{Im}\left(z_{Q}\right)$. Define

$$
H_{Q}(z)=L_{0}(z)+d_{Q}\left(G_{2}(z)-L_{1}(z)\right),
$$

where $L_{0}$ is the unique conformal linear map of $\mathbb{H}$ to itself that maps $z_{Q}$ to $w_{Q}$, and $L_{1}$ is the unique conformal linear map that agrees with $G_{2}$ at $z_{Q}$. Note that $H_{Q}\left(z_{Q}\right)=$ $w_{Q}$ and $\partial L_{0}=d_{Q}$ and that we only need to know the difference $G_{Q}(z)-G_{Q}\left(z_{Q}\right)$ in order to define $H_{Q}(z)$.

By Corollary 35, $\left|\partial G_{2}-\partial L_{1}\right| \leq O(\epsilon)$ on $N_{s}(Q)$. Thus for $z \in N_{s}(Q)$,

$$
\partial H_{Q}=d_{Q}+d_{Q} O(\epsilon)=d_{Q}(1+O(\epsilon)),
$$

and using this we get

$$
\begin{gathered}
\bar{\partial} H_{Q}=d_{Q} \bar{\partial} G_{2}=d_{Q} \mu+O\left(d_{Q} \epsilon^{2}\right)=\left(\mu+O\left(\epsilon^{2}\right)\right) \partial H_{Q}, \\
\mu_{H_{Q}}=\left(\mu+\epsilon^{2}\right)
\end{gathered}
$$

for $z \in Q$. Thus $H_{Q}$ is a quasiconformal map on $N_{S}(Q)$ with dilatation $\mu+O\left(\epsilon^{2}\right)$.

So far we have assumed that we know the images $w_{Q}$ of $z_{Q}$. We now describe how to find $w_{Q}$. We proceed in a "top-down" manner, by starting at the root piece $Q_{0}$ and assuming $H\left(z_{Q_{0}}\right)=z_{Q_{0}}$ is fixed. Then if $Q$ is a child of $Q_{0}$ let $w_{Q}=H_{Q_{0}}\left(z_{Q}\right)$, i.e., use the quasiconformal map for the parent piece to define where the center point of the child maps to. In this way, we can proceed inductively down the tree of pieces and define $H_{Q}$ for every piece.

In each step of this procedure, we might introduce an error of size $O\left(\epsilon^{2}\right)$ in the definition of $w_{Q}$. This is not a problem by itself since we are only trying to compute a map with this accuracy. However, it is possible to have to adjacent pieces $Q, Q^{\prime}$ of our decomposition that have no common ancestor for a very large number of generations, and in such a situation the definitions of $w_{Q}$ and $w_{Q^{\prime}}$ might begin to diverge to an unacceptable degree. In order to avoid this problem, we modify the "top-down" 
induction described above for Whitney type pieces. If the current piece is a Whitney type piece $Q$ and it is adjacent to a Whitney type piece $Q^{\prime}$ of the same height and they have 3rd generation descendants that are Whitney type and adjacent, then we modify the definitions of $w$ for the 3rd generation descendants as follows. For the descendants of $Q$ and $Q^{\prime}$ that are adjacent we define $w$ using the average of the values we would get using $H_{Q}$ and $H_{Q^{\prime}}$. For the descendant next to the center of $Q$, we use the value of $Q$ alone and for the intermediate values we take weighted averages that linearly interpolate between the endpoint cases. This step insures that adjacent pieces have $d_{Q}$ values that are within $1+O(\epsilon)$ of each other, i.e.,

Corollary 36 If $Q$ and $Q^{\prime}$ are adjacent pieces of the decomposition then $d_{Q} / d_{Q^{\prime}}=$ $1+O(\epsilon)$.

Suppose $Q$ and $Q^{\prime}$ are adjacent pieces. How close are the functions $H_{Q}$ and $H_{Q^{\prime}}$ along the common boundary? Note that both maps are quasiconformal on a Carleson square containing both $Q$ and $Q^{\prime}$ and that $H_{Q^{\prime}}$ was chosen to agree with $H_{Q}$ at the point $z_{Q^{\prime}}$. Thus the maps also agree at the reflection of this point in the lower half-plane and both maps take $\infty$ to $\infty$. Moreover, by Corollary 36 and (12), the dilatations of these maps agree to within $\epsilon^{2}$, and hence it follows from Lemma 45 of Appendix A that the maps agree to within $d_{Q} \epsilon^{2}$, i.e.,

$$
\left|H_{Q}(z)-H_{Q^{\prime}}(z)\right|=O\left(d_{Q} \epsilon^{2}\right),
$$

for $z$ in $N_{s}(Q) \cap N_{S}\left(Q^{\prime}\right)$.

Given the approximate solutions $H_{Q}$ on each piece we can combine them using a partition of unity to get a single approximate solution on the whole upper half-plane (again, we only do this to estimate the error; it is not necessary to do so as part of the algorithm). Define a mapping of $\mathbb{H}$ to itself by

$$
H=\sum_{Q} \varphi_{Q} H_{Q},
$$

where $\{\varphi\}$ is a partition of unity as in Sect. 11. If $Q$ and $Q^{\prime}$ are adjacent squares and $z$ is in the $s$ neighborhood of the common boundary, then

$$
\begin{aligned}
& \left|d_{H}\left(z_{Q}\right)-d_{H}\left(z_{Q^{\prime}}\right)\right| \leq C d_{H}\left(z_{Q}\right) \epsilon, \\
& \left|H_{Q}(z)-H_{Q^{\prime}}(z)\right| \leq \epsilon d_{H}\left(z_{Q}\right) \operatorname{Im}\left(z_{Q}\right) .
\end{aligned}
$$

Also,

$$
\bar{\partial} H=\sum_{Q} \bar{\partial} \varphi_{Q} \cdot H_{Q}+\sum_{Q} \varphi_{Q} \cdot \bar{\partial} H_{Q}=I+I I .
$$

Since $\sum_{Q} \bar{\partial} \varphi_{Q}=0, \sum\left|\bar{\partial} \varphi_{Q}\right| \leq C / \operatorname{Im}(z)$, the estimate (13) implies that $I$ is bounded by $O\left(d_{Q} \epsilon^{2}\right)$. Since $\sum_{Q} \varphi_{Q}=1$, the estimate (12) shows that second sum is $d_{Q} \mu+O\left(d_{Q} \epsilon^{2}\right)$. Similarly,

$$
\partial H=\sum_{Q} \partial \varphi_{Q} H_{Q}+\sum_{Q} \varphi_{Q} \partial H_{Q} .
$$


As above, the first sum is $O\left(\epsilon^{2} d_{H}(z)\right)$, and the second sum is

$$
d_{H}\left(z_{Q}\right)\left(1+O\left(\max \left|d_{H}\left(z_{Q}\right)-d_{H}\left(z_{Q^{\prime}}\right)\right|+\epsilon\right)\right)
$$

where the maximum is over all pieces $Q^{\prime}$ that are adjacent to $Q$. This maximum is $O(\epsilon)$, so we have $\partial H=d_{H}\left(z_{Q}\right)(1+O(\epsilon))$ if $z \in Q$. Thus

$$
\mu_{H}(z)=\frac{\bar{\partial} H(z)}{\partial H(z)}=\frac{d_{H}\left(z_{Q}\right)\left(\mu+O\left(\epsilon^{2}\right)\right)}{d_{H}\left(z_{Q}\right)(1+O(\epsilon))}=\mu+O\left(\epsilon^{2}\right) .
$$

Thus in the empty regions (where only one partition of unity function is non-zero) our piecewise solutions $H_{Q}$ agree with a global solution $H$. On the empty regions of the decomposition $H_{Q}$ agrees with a function defined using only $G_{Q}$, which we can compute. Thus the truncated expansions that we can actually compute, agree (on the empty regions) with a globally defined quasiconformal map whose dilatation is $\mu+O\left(\epsilon^{2}\right)$.

\section{Fast Computation of the Beurling Transform}

Now it only remains to compute $\partial G_{Q}(z)-G_{Q}\left(z_{Q}\right) \approx T \mu$ as quickly and as accurately as we claimed. Since we only need to compute this difference in regions where $T \mu$ is holomorphic, we will compute a series expansion for

$$
\partial T \mu(w)=\lim _{r \rightarrow 0} \frac{-1}{\pi i} \iint_{|z-w|>r} \frac{\mu(z)}{(z-w)^{3}} d x d y
$$

and then simply integrate the series term-by-term. We need to compute this with error at most

$$
\frac{C \epsilon^{2}}{\operatorname{diam}\left(Q_{j}\right)}
$$

on a Whitney square $Q_{j}$.

We shall use the fast multipole algorithm of Rokhlin and Greengard [70] (named one of the top ten algorithms of the twentieth century in [39]). The basic idea is that we have $n$ empty regions where want to compute a series expansion, each of which is influenced by the $n$ regions where the data is supported. This is $n^{2}$ interactions to compute in only time $O(n)$. The multipole method takes advantage of the fact that pieces of data that are close together affect distant outputs in similar ways. Thus the data can be grouped together and the combined effects computed simultaneously. By way of review, we first describe how the method works in an easier setting: binary trees.

Suppose $T$ is a binary tree with vertex set $V$ of size $n, f: V \rightarrow \mathbb{R}$ is given, and we want to evaluate the sum

$$
F(v)=\sum_{w \in V \backslash\{v\}} K(v, w) f(w),
$$


at every $v \in V$ where $K(v, w)=a^{\rho_{T}(v, w)}$ and $\rho_{T}$ is the path distance in the tree. There are $n$ inputs and $n$ outputs, and each input affects the evaluation of every output, so naively it seems that $n^{2}$ operations are required. However, all $n$ values of $F$ can be computed in $O(n)$ steps as follows. Choose a root $v_{0} \in V$ and for any $v \in V$ let $D(v) \subset V$ be the vertices that are separated from the root by $v$, not including $v$ (i.e., its descendants). Let $\tilde{D}(v)=V \backslash(\{v\} \cup D(v)$. Let

$$
F_{1}(v)=\sum_{w \in D(v)} K(v, w) f(w), \quad F_{2}(v)=\sum_{w \in \tilde{D}(v)} K(v, w) f(w) .
$$

We can compute each of these functions in one pass through the tree. For $F_{1}$ start by setting $F_{1}(v)=0$ for each leaf of $T$ and proceed from the leaves to the root by setting

$$
F_{1}(v)=a \sum_{w \in C(v)} F_{1}(w),
$$

where the sum is over the children of $v$. This is called the "up-pass" since we start at the leaves and work towards the root. Next, we transfer values from $F_{1}$ to $F_{2}$ using an "across-pass". For each vertex where $F_{1}$ has already been evaluated by the up-pass, add $a^{2} \cdot\left(f(v)+F_{1}(v)\right)$ to $F_{2}(w)$, for each sibling $w \in S(v)$ of $v(w \neq v$ is a sibling of $v$ if it has the same parent as $v$ ). Lastly, we compute $F_{2}$ using a "down-pass", by setting $F_{2}(v)=0$ when $v$ is the root (which has no sibling, so was not affected by the across-pass), and in general if $F_{2}(v)$ has already been computed, then for each of its children $w$ we set

$$
F_{2}(w)=F_{2}(w)+a\left(F_{2}(v)+f(v)\right) .
$$

We get the desired output by noting

$$
F(v)=\left(F_{1}(v)+F_{2}(v)\right),
$$

for every $v \in V$ (we can evaluate vertices in any order). Thus $F$ has been evaluated at all $n$ points in $O(n)$ steps.

The same method works more generally. If we are given a rooted tree $T$ with vertex set $V$ we turn it into a directed graph $G$ by taking two copies $V_{1}, V_{2}$ of $V$, point all edges towards the root in $V_{1}$ and away from the root in $V_{2}$ and connect each vertex in $V_{1}$ to the copies of its siblings in $V_{2}$. Assume we have linear space $X_{v}^{1}, X_{v}^{2}$ for each vertex $v \in V$ and a linear map from each $X_{v}^{1}$ to its parent and from each $X_{v}^{2}$ to each of its children. Assume we also have an "across-map" $A_{v}: X_{v}^{1} \rightarrow X_{v}^{2}$. We define a linear map $L(w, v): X_{w} \rightarrow X_{v}$ by composing maps along the path from $w$ to $v$. Given the $n$ values $x_{v} \in X_{v}^{1}, v \in V$ the method above evaluates all $n$ values of

$$
F\left(x_{v}\right)=\sum_{w \in V} L(w, v) x_{w}
$$

in only $O(n)$ steps.

In the previous example, the linear spaces were one dimensional and the edge maps were multiplication by $a$. For our application to computing a Beurling transform, the tree will be the tree of dyadic Whitney boxes that intersect the support of the dilatation $\mu$. To each Whitney box, $Q$, we will associate a finite set of regions $\left\{W_{j}\right\}$; 
each will be either a disk or a disk complement (including $\infty$ ). The linear spaces will be spaces of analytic functions on these regions. We will actually consider two situations: an infinite dimensional ideal model and a finite dimensional approximation that we actually compute.

In the idealized version, we consider the space $X_{Q}$ of all analytic functions on a region $W$. If $W$ is a disk then every such function has a power series $\sum_{k=0}^{\infty} a_{n}(z-a)^{k}$ converging in the disk and for the disk complements there is a Laurent series $\sum_{k=0}^{\infty} a_{n}(z-a)^{-k}$. The finite dimensional version of these spaces are the spaces $X_{Q}^{p}$. These consist of $p$ term power series $\sum_{k=0}^{p} a_{n}(z-a)^{k}$ (for disks) or Laurent series $\sum_{k=0}^{p} a_{n}(z-a)^{-k}$ (for disk complements). There is an obvious truncation map $T: X_{Q} \rightarrow X_{Q}^{p}$ and an inclusion map $I: X_{Q}^{p} \rightarrow X_{Q}$.

Given two regions, one of which is contained the other, we can restrict a function from the larger region to the smaller. This defines restriction maps $R$ between the infinite dimensional spaces $X_{Q}$. For the finite dimensional analog, we define maps $R^{p}$ between the spaces $X_{Q}^{p}$ by restricting and then truncating the series expansion. We will see how to compute the Beurling transform exactly using the restriction maps, and then check how much error is introduced when we replace these by the finite dimensional restriction/truncation maps.

When we allow infinite expansions, then restricting an analytic function to a subdomain introduces no errors and the method described above allows us to compute series expansion for the Beurling transform in time $O(n)$ with no errors (except for filling in the initial values of the arrays). Similarly, if we restrict a power series to a smaller disk, there is no error introduced, since the restriction of a degree $p$ polynomial is still a degree $p$ polynomial. However, if we change the center of a Laurent expansion, then a finite expansion may become infinite and truncating to $p$ terms causes an error (depending on $p$ and the geometry of the regions). In this case, performing the restriction-truncation along a series of nested regions might not give the same result as restricting to the smallest domain is single step. Because of this, we have to estimate the errors at each step and show the total accumulated error along the whole path is still small.

We will now introduce the elements needed to apply these general ideas to the specific problem of computing $\partial T \mu$.

We start with a partial $\epsilon$-representation of a polygonal domain. As in Sect. 15, we use this to construct a dilatation $\mu$ that is a sum $\sum \mu_{k}$ of terms, each of which are supported in small squares whose union covers a neighborhood, $N_{s}$, of the boundary of our decomposition. We assume that $\mu$ is defined by reflection on the lower halfplane, so that solutions of the Beltrami equation will be real on the real line. Each $\mu_{n}$ is a polynomial in $x$ and $y$ of degree at most $O(n)$ restricted to a small square. The terms of this polynomial are of the form $z^{k} x^{a} y^{b}=(x+i y)^{k} x^{a} y^{b}$ with $0 \leq k \leq p$ and $0 \leq a, b \leq C$ where $p$ grows depending of the desired accuracy, but $C$ is fixed, depending only on the degrees of the piecewise polynomials used in our partition of unity associated to the decomposition $\mathcal{W}$ of our representation. Thus there are only $O(n)$ terms to consider, not $O\left(n^{2}\right)$ as would be the case if all powers of $x$ and $y$ less than $n$ had to be considered.

We take as our tree the collection of all Whitney squares in the upper half-plane that hit the support of $\mu$, i.e., which hit $N_{s}$. There are $O(n)$ such, since there are $O(n)$ 
Fig. 57 A Whitney box and its type I and type II regions
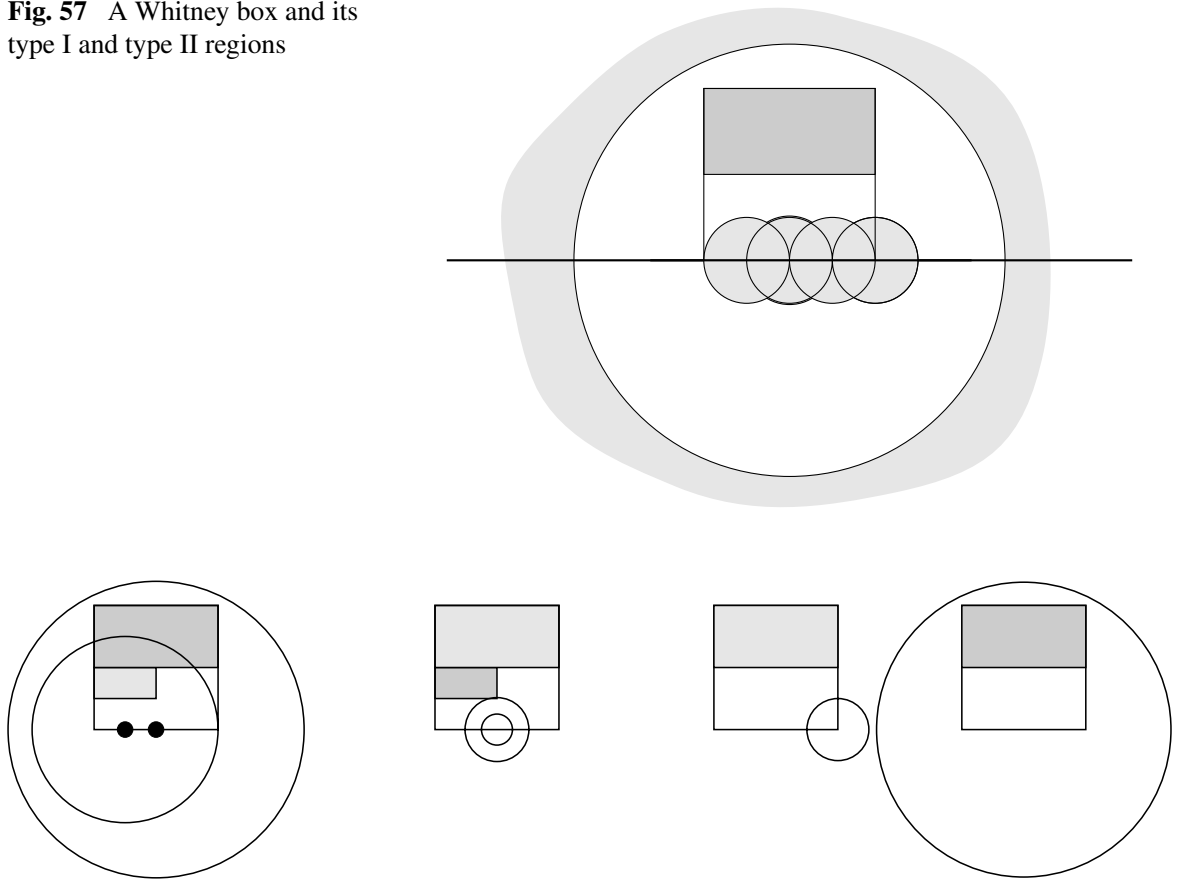

Fig. 58 The three kinds of conversions: multipole-to-multipole, local-to-local and multipole-to-local. In each case, the first Whitney square is shaded lighter than the second

boundary components in our decomposition and each only hits a bounded number of Whitney boxes (for arches we only need to cover the edges of the arch, not the interior). The adjacency relation is the usual one; $Q$ is a child of $Q^{*}$ if the base of $Q^{*}$ contains the base of $Q$ and $Q$ is maximal with this property. A given Whitney square can have zero, one or two children. Those with no children are called "leaves" of the tree. Often a child is half the size of its parent and the top edge of the child is half the bottom edge of the parent, but because of arches, there are some cases where a child is much smaller than its parent. A neighbor of a dyadic Whitney square is a distinct dyadic Whitney square of the same size that touches along the boundary. The terms "descendant" and "ancestor" have the usual meanings for a rooted tree.

For any Whitney box $Q$ in $\mathbb{H}$ with base interval $I$ (its vertical projection on $\mathbb{R}$ ) let $c_{Q}$ denote the center of this base and let $c_{Q}^{j}, j=1, \ldots, 8$, be 8 equally spaced points in $I$ (including the right, but not the left endpoint of I). Let $A_{Q}=\left\{z:\left|z-c_{Q}\right| \geq \lambda|I|\right\}$ where we choose $\frac{1}{2} \sqrt{5}<\lambda<\frac{5}{4}$, and let $D_{Q}^{j}=\left\{z:\left|z-c_{Q}^{j}\right| \leq \frac{1}{4}|I|\right\}$. These will be called the type I and type II regions associated to $Q$, respectively; see Fig. 57 . The number $\lambda$ is chosen in this range so that the type I region does not intersect $Q$, but it does contain the type II regions of any $Q^{\prime}$ that is the same size as $Q$, but not adjacent to it; see Fig. 58. A series expansion in terms of $\left(z-c_{Q}\right)^{-1}$ or $\left(z-c_{Q}^{j}\right)$ will be called type I and type II expansions, respectively.

Given a Whitney box $Q$, we can restrict $\mu$ to $Q$ and compute type I and type II expansions for $\left.\partial T \mu\right|_{Q}$. Since $\mu$ is a piecewise polynomial of degree $O(p)$, and there 
is an explicit formula for the expansion of each monomial, this can be done in time $O(p \log p)$ by the remarks in Appendix B.

Given a Whitney box $Q$ and its parent $Q^{*}$, the type I region for $Q$ contains that for $Q^{*}$ and so we can take the analytic function $f$ defined by the type I expansion for $Q$ and compute its Laurent expansion in the type I region for $Q^{*}$. Then truncate this (infinite) series to get a type I expansion for $Q^{*}$. This is called a I-to-I conversion or a multipole-to-multipole conversion; see Fig. 58. This introduces an error of $\epsilon M_{f}$, where $\epsilon=\lambda^{p}$, where $\lambda<1$ and $M_{f}$ is the maximum of $f$ on the type I region of $Q$.

Similarly, we can take a type II expansion for $Q$ and restrict it to one of the two type II regions for a child of $Q$ whose center agrees with the first center or is immediately to the left of it. Changing the center of the expansion just gives another degree $p$ polynomial and there is no error introduced, i.e., $R=R^{p}$. This is a local-to-local conversion.

Finally, the type I region of a Whitney box $Q$ contains the type II regions of a box $Q^{\prime}$ of the same size if $Q^{\prime}$ is in $3 Q^{*}$ but not in $3 Q$ (here $Q^{*}$ denotes the parent of $Q$ ). Therefore, we can restrict the type I expansion of $Q$ to a type II region of $Q^{\prime}$ and do a I-to-II conversion (or multipole-to-local conversion), with an error of $\epsilon M_{f}$, as above.

For a given box $Q$, the associated regions cover the whole upper half plane, except for a region of bounded hyperbolic diameter around $Q$.

We think of the type I and type II expansions associated to each $Q$ as defining two arrays indexed by the Whitney boxes. We next describe how to initialize and update these arrays:

Initialize Type I array For each $Q$ compute the initial type I expansion.

Initialize Type II array For each $Q$ compute the initial type II expansion for each type II disk.

Modify Type II array Compute type II expansions for two neighbors and add to their initial expansions. Every type II expansion now has contributions from at most three boxes (its parent and the parent's neighbors).

Perform the up-pass Starting with leaves of the tree, do I-to-I conversion of the current type I expansion and add it to the type I expansion of the parent. Continue until we reach the root.

Perform the across-pass For each square $Q$, do I-to-II conversions taking the current type I expansion and obtaining type II conversions for regions corresponding to centers in $3 I^{*} \backslash 3 I$ where $I$ is the base of $Q$ and $I^{*}$ is the base of $Q$ 's parent.

Perform the down-pass Starting with root square, do II-to-II conversions, taking each type II expansion and restricting it to the two type II expansions of the children. Continue downward until we reach the leaves of the tree.

This is clearly $O(n)$ steps and when we are finished, the type I expansion of a square $Q$ contains the contribution of $Q$ and every descendant of $Q$ and the type II expansions of $Q$ contain the contributions of every square that is not a (strict) descendant of $Q$ or its two neighbors.

The third step (Modify the type II array) is necessary because the tree structure on Whitney squares does not completely reflect their actual placement in $\mathbb{H}$; two squares that are far apart in the tree could be adjacent in $\mathbb{H}$. The type I region of a square does not contain the type II disks of its neighbors (they are too close), so we need this 
special step to pass the information to these regions (for other regions it is passed in the across-step).

Now suppose $D$ is an empty piece of our decomposition and $Q$ is the Whitney box containing $D$. If $D$ is a disk, it is contained in a type II region of a grandparent of $Q$ and is contained in the type I region of all grandchildren of $Q$ and its two neighbors. Therefore, we can do series conversions and compute the expansion in $D$ due to these expansions. There are only a finite number of Whitney boxes whose contributions have not been accounted for and all these lie within a uniformly bounded distance of $Q$. For each piece of $\mu$ supported in one of these boxes, we compute the contribution to $D$ directly.

Given any Whitney boxes $Q^{\prime}$ and $Q$ there is a path in our directed graph that starts from an initial expansion for $Q$ and goes to a terminal expansion for $Q^{\prime}$ or one of its neighbors. To see this we consider several cases:

(1) If $Q=Q^{\prime}$ there is nothing to do.

(2) If $Q=Q_{2}$ is an ancestor of $Q_{1}$ then the all "down" path works.

(3) If $Q_{3}$ is a neighbor of square $Q_{2}$ in (1), then start with the special "Modify type II" step and follow by all downs.

(4) If $Q_{4}$ is a descendant of a case (2) square, then follow "up" paths until we hit a child of $Q_{3}$ and then use a "across" step to bring us to an ancestor of $Q^{\prime}$ (this works because by the definition of the across step).

(5) If $Q$ is a descendant of $Q^{\prime}$, then use all "up"s.

(6) The only remaining case is that $Q$ is a neighbor of $Q^{\prime}$ or a descendant of a neighbor. Using an all "up" path works.

If $D$ is an empty piece of our decomposition, we want to show that the desired expansion for $\partial T \mu$ can be computed using a bounded number or type I and type II expansions, plus a bounded number of direct expansions of nearby squares. Fix such a $D$ and suppose $Q$ is any Whitney box, then $D$ is a subset of one of the type I or II regions associated to $Q$ or one of its neighbors, unless $Q$ is within a uniformly bounded hyperbolic distance $M$ of $D$. If $D$ is a subset of one of these regions, then we can convert the series expansion on the region to one on $D$, the conversion being one of three types. First, we might have to convert a power series in $(z-a)$ to one in $(z-b)$; this happens when $D$ is a disk or arch contained in a disk and involves no loss of accuracy. Second, converting an expansion in $(z-a)^{-1}$ to one in $(z-b)^{-1}$; this happens when $D$ is an arch that contains $Q$ in its bounded complementary component and there is a loss of accuracy (described in Lemma 37). Finally, in all other cases we must convert an expansion in $(z-a)^{-1}$ to one in $(z-b)$; this also involves a small loss of accuracy.

Lemma 37 Suppose $|a| \leq \frac{1}{4},|b| \leq \frac{1}{2}$ and $f$ is analytic on $\{z:|z-a|>1\}$ and $|f| \leq 1$ there. Also assume $f(z) z^{3}$ is bounded as $z \rightarrow \infty$. Suppose $A=$ $\{z:|z-b|>2\}$ and let $f(z)=\sum_{j=0}^{\infty} a_{j}(z-b)^{-j}$ be the Laurent expansion for $f$ in $A$ and let $g(z)=\sum_{j=0}^{p} a_{j}(z-b)^{-j}$. Then there is $0<\lambda<1$ so that for $\epsilon=\lambda^{p}$,

(1) $a_{0}=a_{1}=a_{2}=0$,

(2) $|g(z)| \leq(1+\epsilon)|z|^{-3} \leq|z|^{5 / 2}$, if $p$ is large enough,

(3) $|f(z)-g(z)| \leq \epsilon|z|^{-3}$. 
The proof is just the standard estimates for Taylor series and left to the reader. If we start with an expansion $f_{0}$ on the type I region of a box $Q$ of size 1 and then restrict the expansion to get an expansion $f_{1}$ for the type I region of its parent, then repeat this over and over, we accumulate an error each time. Suppose $f_{k}$ is $k$ th expansion on the $k$ th region $A_{k}$. Then $\sup _{A_{k}}\left|f_{k}\right| \leq|z|^{-5 / 2}$, which means the maximum error between $f_{k}(z)$ and $f_{k+1}(z)$ on $A_{k+1}$ is bounded by $\epsilon|z|^{-5 / 2}$. So the total error that is ever possible inside $A_{N}$ is

$$
\begin{aligned}
& \leq \sum_{k=1}^{N}\left|f_{k}(z)-f_{k+1}(z)\right| \\
& \leq \sum_{k=1}^{N} \epsilon \operatorname{diam}\left(\partial A_{k}\right)^{-5 / 2}\left(\operatorname{diam}\left(\partial A_{n}\right) / \operatorname{diam}\left(\partial A_{k}\right)\right)^{-3} \\
& \leq O(\epsilon) \operatorname{diam}\left(\partial A_{N}\right)^{-3} \sum_{k=1}^{N} \operatorname{diam}\left(\partial A_{k}\right)^{1 / 2} .
\end{aligned}
$$

Since the regions grow by at least a factor of two at each stage, the final sum is dominated by its final term, and so the total error is less than $O\left(\epsilon \operatorname{diam}\left(\partial A_{N}\right)^{-2.5}\right)$.

Summarizing this argument gives:

Lemma 38 Suppose $f_{0}(z)=\sum_{k=3}^{p} a_{k}\left(z-c_{Q}\right)^{-k}$ is a type I expansion associated to a dyadic Whitney square $Q_{0}$ and $\left|f_{0}\right|$ is bounded by $M$ on the type I region of $Q_{0}$. Suppose $Q_{1}, \ldots, Q_{N}$ are ancestors of $Q$ and $f_{k}$ is the result of applying a I-to-I conversion to $f_{k-1}$ for $k=1, \ldots, N$. Then on the type I region of $Q_{N},\left|f_{0}-f_{N}\right| \leq$ $O\left(\epsilon M\left(\operatorname{diam}\left(Q_{0}\right) / \operatorname{diam}\left(Q_{N}\right)\right)^{2.5}\right)$ with $\epsilon=\lambda^{p}$ and a constant that is independent of $N$.

Corollary 39 Suppose $Q_{0}$ is a Whitney square and for each $Q$ that is a descendant of $Q_{0}$, let $f_{Q}$ be the type I expansion of $\partial T \mu$ for $\mu$ restricted to $Q$. Let $f_{0}$ be the type I expansion for $Q_{0}$ obtained by running the up-pass over all descendants of $Q$ with initial data $\left\{f_{Q}\right\}$. Let $F_{0}=\sum_{Q \in D\left(Q_{0}\right)} f_{Q}$ be the exact sum of these initial expansion restricted to the type I region of $Q_{0}$. Then

$$
\left|f_{0}(z)-F_{0}(z)\right|=O\left(\epsilon \frac{\operatorname{diam}\left(Q_{0}\right)^{2}}{\left|z-c_{Q_{0}}\right|^{3}}\|\mu\|_{\infty}\right) .
$$

Proof The maximum of $f_{Q}$ on the type I region of $Q$ is clearly $O\left(\|\mu\|_{\infty} / \operatorname{diam}(Q)\right)$. Therefore, by the Lemma 38 the error of applying I-to-I conversions until we reach $Q_{0}$ is

$$
O\left(\epsilon\|\mu\|_{\infty} \operatorname{diam}(Q)^{1.5} / \operatorname{diam}\left(Q_{0}\right)^{2.5}\right) .
$$

There are at most $2^{k}$ descendants of $Q_{0}$ with $\operatorname{diam}(Q)=2^{-k} \operatorname{diam}\left(Q_{0}\right)$, so the total error of all of these is

$$
O\left(\epsilon\|\mu\|_{\infty} \operatorname{diam}(Q)^{0.5} / \operatorname{diam}\left(Q_{0}\right)^{1.5}\right)=O\left(\epsilon\|\mu\|_{\infty} 2^{-k / 2} / \operatorname{diam}\left(Q_{0}\right)\right) .
$$


We now sum over $k=1,2, \ldots$ and see the total error over all descendants of $Q_{0}$ is at most $O\left(\epsilon\|\mu\|_{\infty} / \operatorname{diam}\left(Q_{0}\right)\right)$. The error is an analytic function on the type I region of $Q_{0}$ that decays like $\left|z-c_{Q_{0}}\right|^{-3}$ near infinity (since it is a difference of functions which do), and this gives the estimate in the corollary.

Corollary 40 Let $f$ be a type II expansion for a Whitney square $Q$ that is obtained by starting with expansions of $\partial T \mu$ and applying the up-pass, across-pass and downpass. Then the total error between $f$ and simply adding all the initial expansions that contribute to $f$ is $O\left(\epsilon\|\mu\|_{\infty} / \operatorname{diam}(Q)\right)$.

Proof The contribution of the ancestors of $Q$ are through II-to-II conversions, which introduce no error. The neighbors of ancestors contribute are direct computation of a type II expansion, followed by II-to-II expansions, so also contribute no error. Every other contribution comes from a sequence of I-to-I conversions (the uppass), followed by a I-to-II conversion (the across-pass) and then II-to-II conversions (the down-pass). Fix a square $Q_{j}$ to which the across-pass is applied. The errors due to all the descendants of $Q_{j}$ is bounded by $O\left(\epsilon\|\mu\|_{\infty} / \operatorname{diam}\left(Q_{j}\right)\right)$. If $\operatorname{diam}\left(Q_{j}\right)=2^{j} \operatorname{diam}(Q)$, then this is $O\left(2^{-j} \epsilon\|\mu\|_{\infty} / \operatorname{diam}(Q)\right)$. The across-pass adds an error with the same bound and the following down-pass adds no new error. Thus the total contribution of $Q_{j}$ to the error is $O\left(2^{-j} \epsilon\|\mu\|_{\infty} / \operatorname{diam}(Q)\right)$. There are only a bounded number (at most 4 ) of such $Q_{j}$ 's of a given size, so summing over all possible $j$ 's shows the total error is at most $O\left(\epsilon\|\mu\|_{\infty} / \operatorname{diam}(Q)\right)$.

Now every expansion that contributes to the final expansion on an empty region is either directly computed from the data, or comes from an expansion created by the up-pass, across-pass and down-pass. By the corollary, the up-pass creates a small error, and we already know the across-pass creates a small error and the down-pass creates no errors. Thus the total error comes from a uniformly bounded number of terms, each of which has error bounded by $O\left(\epsilon\|\mu\|_{\infty} / \operatorname{diam}(Q)\right)$.

This completes the proof of Lemma 31 and hence of the theorem.

\section{Appendix A: Background in Analysis}

In this section, will give various definitions and results from analysis. It is intended as a review or (very brief) introduction to ideas used in the paper.

\section{A.1 Möbius Transformations}

A linear fractional (or Möbius) transformation is a map of the form $z \rightarrow$ $(a z+b) /(c z+d)$. This is a $1-1$, onto, holomorphic map of the Riemann sphere $\mathbb{S}=\mathbb{C} \cup\{\infty\}$ to itself. Such maps form a group under composition and are well known to map circles to circles (if we count straight lines as circles that pass through $\infty$ ). Möbius transforms are conformal, so they preserve angles.

The non-identity Möbius transformations are divided into three classes. Parabolic transformations have a single fixed point on $\mathbb{S}$ and are conjugate to the translation 
map $z \rightarrow z+1$. Elliptic maps have two fixed points and are conjugate to the rotation $z \rightarrow \lambda z$ for some $|\lambda|=1$. The loxodromic transformations also have two fixed points and are conjugate to $z \rightarrow \lambda z$ for some $|\lambda|<1$. If, in addition, $\lambda$ is real, then the map is called hyperbolic.

Given two sets of three distinct points $\left\{z_{1}, z_{2}, z_{3}\right\}$ and $\left\{w_{1}, w_{2}, w_{3}\right\}$ there is a unique Möbius transformation that sends $w_{k} \rightarrow z_{k}$ for $k=1,2,3$. A Möbius transformation sends the unit disk 1-1, onto itself iff it is of the form

$$
z \rightarrow \lambda \frac{z-a}{1-\bar{a} z}
$$

for some $a \in \mathbb{D}$ and $|\lambda|=1$. Any loxodromic transformation of this form must actually be hyperbolic.

\section{A.2 Conformal Maps}

A conformal mapping is a diffeomorphism that preserves angles. We will only consider orientation preserving maps here, in which case a conformal map between planar domains is the same as a 1-1 holomorphic mapping. The Riemann mapping theorem states that given any simply connected, proper subdomain $\Omega$ of the plane there is a one-to-one, onto holomorphic map $f: \mathbb{D}=\{z:|z|<1\} \rightarrow \Omega$. If $\partial \Omega$ is locally connected then this map extends continuously to the boundary. Moreover, we can always take $f(0)$ to be any given point of $\Omega$ and $f^{\prime}(0)$ to have any argument we want. If $\partial \Omega$ is a Jordan curve we can also normalize by making any three points on the unit circle map to any three points on $\partial \Omega$ (as long as they have the same orientation).

The Schwarz-Christoffel formula gives a formula for the Riemann map of the disk onto a polygonal region $\Omega$ : if the interior angles of $P$ are $\alpha \pi=\left\{\alpha_{1} \pi, \ldots, \alpha_{n} \pi\right\}$, then

$$
f(z)=A+C \int^{z} \prod_{k=1}^{n}\left(1-\frac{w}{z_{k}}\right)^{\alpha_{k}-1} d w ;
$$

see, e.g., $[48,98,126]$. On the half-plane, the formula is

$$
f(z)=A+C \int \prod_{k=1}^{n-1}\left(w-z_{k}\right)^{\alpha_{k}-1} d w .
$$

The formula was discovered independently by Christoffel in 1867 [38] and Schwarz in 1869 [109, 110]. For other references and a brief history, see Sect. 1.2 of [48]. It is also possible to formulate it with other base domains, such as an infinite strip (see [48]). See [82] for a version involving doubly connected polygonal regions. There are also versions for domains other than polygons, e.g., circular arc polygons as in $[81,98]$. In this case, we get a simple formula for the Schwarzian derivative of the conformal map, but it involves unknown parameters with no obvious geometric interpretation.

One particular case of the Schwarz-Christoffel formula we need (see Sect. 11) is for the map of the upper half-plane to a triangle with one vertex at $\infty$ (i.e., a region bounded by two half-infinite rays and a finite segment). Since there are only two finite 
vertices, there is no parameter problem to solve; they can be chosen to be any two points we want, say \pm 1 , so the formula becomes

$$
f(z)=A+C \int(w-1)^{\alpha_{1}-1}(w+1)^{\alpha_{2}-1} d w .
$$

Note that using the general form of the binomial theorem,

$$
(1+z)^{p}=\sum_{k=0}^{\infty} \frac{p(p-1) \cdots(p-k+1)}{k !} z^{k},
$$

we can easily compute power series for these functions in disks away from the singularities.

The problem in applying the Schwarz-Christoffel formula with $n>3$ vertices is that the points $\mathbf{z}=\left\{z_{1}, \ldots, z_{n}\right\}$ are unknown to us until we know $f$, so the formula seems circular. However, there are various iterative methods for finding the points $\mathbf{z}$ starting from an initial guess (often taken to be $n$ uniformly distributed points on $\mathbb{T}$ ), e.g., see [48, 87]. For example, the method of Davis [45] takes an $n$-tuple of points $\left\{z_{1}, \ldots, z_{n}\right\}$ on the unit circle, computes an image polygon using the SchwarzChristoffel formula with these parameters (and the known angles) and compares the side lengths of this polygon with the desired polygon. If a side is too short, the corresponding parameter values are moved apart in the next iteration and conversely. More precisely, if $\left\{z_{1}^{k}, \ldots, z_{n}^{k}\right\}$ is the current guess, and the image polygon has vertices $\left\{v_{1}^{k}, \ldots, v_{n}^{k}\right\}$ we define the next set of parameter guesses as

$$
\left|z_{k}^{j+1}-z_{j-1}^{k+1}\right|=k\left|z_{j}^{k}-z_{j-1}^{k}\right| \frac{\left|v_{j}-v_{j-1}\right|}{\left|v_{j}^{k}-v_{j-1}^{k}\right|},
$$

for $j=0, \ldots, n$, where $k$ is a normalizing constant and $\mathbf{v}=\left\{v_{0}, \ldots, v_{n}\right\}$ are the vertices of the target polygon. The method works in practice in many cases but is not known to converge.

Davis' method is used in [7] by Banjai and Trefethen to give a $O(n)$ method for finding the prevertices that is practical for tens of thousands of vertices (the bound, however, is an average case analysis, not a uniform estimate for all polygons). Many other methods exist for computing conformal mappings including integral equation methods that are very effective. For example, in [99] Rokhlin and O'Donnell compute conformal maps using the fast multipole method to solve an integral equation arising from the Kerzman-Stein formula. Marshall has a fast method called "zipper" based on iterating simple maps (see [93]). For surveys of different numerical conformal mapping techniques, see, e.g., [46, 63, 75, 83, 101, 125, 129, 132].

A circle packing of a domain is a collection of disjoint (except for tangencies) disks in the domain. The Andreev-Thurston theorem say that given such a packing one can find a packing of the disk with the same tangency relations and that if the circles are small enough the mapping between the packings is an approximation to the Riemann map [72, 73, 108, 117-119]. A polynomial time algorithm for computing conformal mappings is described in [115] using a polynomial time algorithm for finding circle packings, but no details are provided about how to choose a packing of a domain in time independent of the geometry. A polynomial time algorithm for 
circle packings is also described in $[96,97]$. Software for computing conformal maps via circle packings is available from Ken Stephenson [116].

An alternate approach to the computational complexity of conformal mapping is considered by Binder, Braverman and Yampolsky in [10]. They consider domains with complicated boundaries (such a fractals) and assume an oracle is given that will decide whether a given point is within $\epsilon$ of the boundary. The complexity of a domain is determined by how quickly such an oracle works (as a function of $\epsilon$ ). Given such an oracle they show the Riemann mapping at a point can be computed to accuracy $\epsilon^{a}$ using $b \log ^{2} \epsilon$ space and $\epsilon^{-c}$ time for some positive constants $a, b, c$ (their method solves a Dirichlet problem using a random walk on an $\epsilon$-grid stopped by the oracle). This is related to other notions of the computability of conformal maps, such as constructibility in the sense of Brouwder and Errett Bishop, e.g., see $[18,31,76,138]$.

\section{A.3 Hyperbolic Geometry}

On the unit ball, $\mathbb{B}$, the hyperbolic metric is given by

$$
|d \rho|=\frac{2|d z|}{1-|z|^{2}} .
$$

More explicitly, it can be written as

$$
\rho\left(z_{1}, z_{2}\right)=\frac{1}{2} \log \frac{1+\left|\sigma\left(z_{2}, z_{1}\right)\right|}{1-\left|\sigma\left(z_{2}, z_{1}\right)\right|},
$$

where $\sigma\left(z_{1}, z_{2}\right)=\left(z_{2}-z_{1}\right) /\left(1-\bar{z}_{1} z_{2}\right)$. In the upper half space model, $\mathbb{R}_{+}^{3}$, it is given by

$$
|d \rho|=\frac{|d z|}{\operatorname{dist}\left(z, \partial \mathbb{R}^{2}\right)} .
$$

For the ball and upper half-space models, hyperbolic geodesics are circular arcs that are orthogonal to the boundary (also vertical lines in the case of the half-space).

Hyperbolic area on $\mathbb{D}$ is defined as $d x d y /\left(1-|z|^{2}\right)^{2}$ and on $\mathbb{H}$ as $d x d y / y^{2}$. The disk and half-plane have infinite area; indeed, each Whitney square (Sect. 9) has area $\simeq 1$ and the area of a hyperbolic $r$-ball is grows exponentially with $r$. A striking feature of hyperbolic geometry is that the area of a triangle is determined by its three angles, namely area $(T)=\pi-(\alpha+\beta+\gamma)$. Thus an ideal triangle (one with all three vertices on the boundary) has area $\pi$ and all other triangles have smaller area.

Simply connected, proper subdomains of the plane inherit a hyperbolic metric from the unit disk via the Riemann map. If $\varphi: \mathbb{D} \rightarrow \Omega$ is conformal and $w=\varphi(z)$ then $\rho_{\Omega}\left(w_{1}, w_{2}\right)=\rho_{\mathbb{D}}\left(z_{1}, z_{2}\right)$ defines the hyperbolic metric on $\Omega$ and is independent of the particular choice of $\varphi$. It is often convenient to estimate $\rho_{\Omega}$ in terms of the more geometric "quasi-hyperbolic" metric on $\Omega$ that is defined as

$$
\tilde{\rho}\left(w_{1}, w_{2}\right)=\inf \int_{w_{1}}^{w_{2}} \frac{|d w|}{\operatorname{dist}(w, \partial \Omega)},
$$

where the infimum is over all arcs in $\Omega$ joining $w_{1}$ to $w_{2}$. 
Theorem 41 (Koebe's distortion theorem) Suppose $\varphi: \mathbb{D} \rightarrow \Omega$ is a conformal map of the disk to a simply connected domain. Then for all $z \in \mathbb{D}$,

$$
\frac{1}{4}\left|\varphi^{\prime}(z)\right|\left(1-|z|^{2}\right) \leq \operatorname{dist}(\varphi(z), \partial \Omega) \leq\left|\varphi^{\prime}(z)\right|\left(1-|z|^{2}\right) .
$$

Because of Koebe's distortion theorem, we have

$$
d \rho_{\Omega} \leq d \tilde{\rho}_{\Omega} \leq 4 d \rho_{\Omega}
$$

Lemma 42 Suppose $\Omega$ is simply connected and $z \in \Omega$ satisfies $\operatorname{dist}(z, \partial \Omega)=1$. If either $|w-z|>R$ or $\operatorname{dist}(w, \partial \Omega)<1 / R$, then $\rho_{\Omega}(z, w) \geq \frac{1}{4} \log R$.

Proof Recall that $\rho_{\Omega} \geq \frac{1}{4} \tilde{\rho}$ where $\tilde{\rho}$ denotes the quasi-hyperbolic metric on $\Omega$, defined by $d \tilde{\rho}=|d z| / \operatorname{dist}(z, \partial \Omega)$. If $\operatorname{dist}(w, \partial \Omega) \leq 1 / R$ then $\tilde{\rho}(z, w) \geq \int_{1 / R}^{1} \frac{1}{t} d t=$ $\log R$. If $|w-z| \geq R$, then $\tilde{\rho}(z, w) \geq \int_{1}^{R} \frac{1}{t} d t \geq \log R$.

Möbius transformations are the only 1-1, onto holomorphic maps of the Riemann sphere to itself. In the complex plane, we write these maps as $z \rightarrow(a z+b) /(c z+d)$. Every such map extends uniquely to be a hyperbolic isometry of the upper half-space, $\mathbb{R}_{+}^{3}$, and every orientation preserving isometry on $\mathbb{R}_{+}^{3}$ is of this form.

\section{A.4 Conformal Modulus}

Suppose $\Gamma$ is a family of locally rectifiable paths in a planar domain $\Omega$ and $\rho$ is a non-negative Borel function on $\Omega$. We say $\rho$ is admissible for $\Gamma$ if

$$
\ell(\Gamma)=\inf _{\gamma \in \Gamma} \int_{\gamma} \rho d s \geq 1
$$

and define the modulus of $\Gamma$ as

$$
\operatorname{Mod}(\Gamma)=\inf _{\rho} \int_{\Omega} \rho^{2} d x d y
$$

where the infimum is over all admissible $\rho$ for $\Gamma$. The reciprocal of the modulus is called the extremal length of the path family. These are important conformal invariants whose basic properties are discussed in many sources such [2]. A simple result we used in this paper is:

Lemma 43 Suppose $\gamma \subset \mathbb{D}$ is a Jordan arc with one endpoint on $\partial \mathbb{D}$ and has diameter $\leq \frac{1}{4}$. Then the modulus of the path family separating $\gamma$ from $B\left(0, \frac{1}{2}\right)$ in $\mathbb{D}$ is $\pi / \log (\operatorname{diam}(\gamma))+O(1)$.

A generalized quadrilateral $Q$ is a Jordan domain in the plane with four specified boundary points $x_{1}, x_{2}, x_{3}, x_{4}$ (in counterclockwise order). We define the modulus 
of $Q, M_{Q}\left(x_{1}, x_{2}, x_{3}, x_{4}\right)$ (or just $M_{Q}$ or $M(Q)$ if the points are clear from context), as the modulus of the path family in $Q$ that connects the $\operatorname{arc}\left(x_{1}, x_{2}\right)$ to the arc $\left(x_{3}, x_{4}\right)$. This is also the unique positive real number $M$ such that $Q$ can be conformally mapped to a $1 \times M$ rectangle with the arcs $\left(x_{1}, x_{2}\right),\left(x_{3}, x_{4}\right)$ mapping to the opposite sides of length 1 . Given a generalized quadrilateral $Q$ with four boundary points $x_{1}, x_{2}, x_{3}, x_{4}$, the quadrilateral $Q^{\prime}$ with vertices $x_{2}, x_{3}, x_{4}, x_{1}$ is called the reciprocal of $Q$ and it is easy to see that $\operatorname{Mod}\left(Q^{\prime}\right)=1 / \operatorname{Mod}(Q)$. We also call $\operatorname{Mod}\left(Q^{\prime}\right)$ the "extremal distance" from $\left(x_{1}, x_{2}\right)$ to the $\operatorname{arc}\left(x_{3}, x_{4}\right)$ in $Q$.

\section{A.5 Cross-Ratio}

Given four distinct points $a, b, c, d$ in the plane we define their cross-ratio as

$$
\operatorname{cr}(a, b, c, d)=\frac{(d-a)(b-c)}{(c-d)(a-b)} .
$$

Note that $\operatorname{cr}(a, b, c, z)$ is the unique Möbius transformation that sends $a$ to $0, b$ to 1 , and $c$ to $\infty$. This makes it clear that cross-ratios are invariant under Möbius transformations; that $\operatorname{cr}(a, b, c, d)$ is real valued iff the four points lie on a circle; and is negative iff in addition the points are labeled in counterclockwise order on the circle. If the four points lie on $\mathbb{T}$, then since cr and $M_{\mathbb{D}}$ are both invariant under Möbius transformations of the disk to itself, each must be a function of the other in this case. The function is explicitly given as in infinite product in Ahlfors' book [2].

\section{A.6 Quasiconformal Mappings}

Quasiconformal mappings are a generalization of conformal mappings that play an important role in modern analysis and a central role in the current paper. There are (at least) three equivalent definitions of a $K$-quasiconformal mapping between planar domains. Suppose $f: \Omega \rightarrow \Omega^{\prime}$ is a homeomorphism. We say $f$ is $K$-quasiconformal if any of the following equivalent conditions holds:

Geometric definition for any generalized quadrilateral $Q \subset \Omega, \operatorname{Mod}(Q) / K \leq$ $\operatorname{Mod}(f(Q)) \leq K \operatorname{Mod}(Q)$.

Analytic definition $f$ is absolutely continuous on almost every vertical and horizontal line and the partial derivatives of $f$ satisfy $\left|f_{\bar{z}}\right| \leq k\left|f_{z}\right|$ where $k=(K-1) /$ $(K+1)$.

Metric definition For every $x \in \Omega$

$$
\limsup _{r \rightarrow 0} \frac{\max _{y:|x-y|=r}|f(x)-f(y)|}{\min _{y:|x-y|=r}|f(x)-f(y)|} \leq K .
$$

For a proof of the equivalence of the first two, see [2]; and for a discussion of the third and a generalization to metric spaces, see [74] and its references. In Euclidean space, the equivalence of the three definitions is due to Gehring [66, 67]. A composition of a $K_{1}$-quasiconformal map with a $K_{2}$-quasiconformal map is $\left(K_{1} K_{2}\right)$-quasiconformal. Thus the distance used in Theorem 2 satisfies the triangle inequality. 
A closed curve in the plane is called a $K$-quasicircle if it is the image of a circle under a $K$-quasiconformal homeomorphism of the plane and is called a quasicircle if it is a $K$-quasicircle for some $K<\infty$. Quasicircles have a geometric characterization in terms of Ahlfors' three point condition: a curve $\gamma$ is a quasicircle iff for any two points $x, y \in \gamma$ one of the two arcs with endpoints $x, y$ has diameter $\leq M|x-y|$. Quasicircles need not be differentiable; a famous example of a non-differentiable quasicircle is the von Koch snowflake.

Recall that $\partial f=f_{z}=\frac{1}{2}\left(f_{x}-i f_{y}\right)$ and $\bar{\partial} f=f_{\bar{z}}=\frac{1}{2}\left(f_{x}+i f_{y}\right)$. For a $K$ quasiconformal map the ratio $\mu_{f}=f_{\bar{z}} / f_{z}$ is a well defined complex function almost everywhere and satisfies $\left\|\mu_{f}\right\|_{\infty} \leq k=(K-1) /(K+1)$. The function $\mu=\mu_{f}$ is called the Beltrami coefficient of $f$ and satisfies the following composition laws:

$$
\begin{aligned}
\mu_{f^{-1}} \circ f & =-\left(f_{z} / \overline{f_{z}}\right)^{2} \mu_{f}, \\
\mu_{g \circ f}(z) & =\left(f_{z}(z) / \bar{f}_{z}(z)\right) \frac{\mu_{g}(f(z))-\mu_{f}(z)}{1-\mu_{g}(f(z)) \overline{\mu_{f}(z)}} .
\end{aligned}
$$

If a conformal map of the plane to itself fixes two points, it must be the identity. A $(1+\epsilon)$-quasiconformal map of fixing two points must be close to the identity in the following sense.

Lemma 44 There is $a 0<k<1$ and $a C<\infty$ so that the following holds. Suppose that $f$ is a quasiconformal mapping of the plane to itself that preserves $\mathbb{H}$, fixing 0,1 and $\infty$ and the Beltrami coefficient of $f$ is $\mu$ with $\|\mu\|_{\infty} \leq k$. Then

$$
\left|f(w)-\left[w-\frac{1}{\pi} \int_{\mathbb{R}^{2}} \mu(z) R(z, w) d x d y\right]\right| \leq C\|\mu\|_{\infty}^{2},
$$

for all $|w| \leq 1$, where

$$
R(z, w)=\frac{1}{z-w}-\frac{w}{z-1}+\frac{w-1}{z}=\frac{w(w-1)}{z(z-1)(z-w)} .
$$

This precise statement [12, Lemma 2.6] is based on a similar result in [2, Sect. V.C]. If $|\mu|$ is bounded by $\epsilon$ then since $R$ is integrable, this says $|f(w)-w|=O(\epsilon)$ as long as $|w|$ is bounded. From this one can deduce

Lemma 45 Suppose $f: \mathbb{D} \rightarrow \mathbb{D}$ is $(1+\epsilon)$-quasiconformal and that it fixes the boundary points $1,-1, i$. Then $\sup _{x \in \mathbb{T}}|f(x)-x|=O(\epsilon)$.

This justifies our claim in the introduction that if we can approximate the prevertices in the QC-sense, then we have also approximated them in the uniform sense. We will also use the estimate:

Lemma 46 Suppose $f$ is a conformal mapping on $\mathbb{D}$ such that $f(0)=0, f^{\prime}(0)=1$ and $f$ has a $(1+\epsilon)$-quasiconformal extension to the plane fixing $\infty$. Then for $0 \leq$ $r<1, \sup _{|z| \leq r}\left|f^{\prime}(z)-1\right|=O\left(\frac{\epsilon}{1-r}\right)$. 
To prove this, we use (10) of [2, Sect. V.B], which implies that for a $(1+\epsilon)$-QC map $f$ fixing 0 and $\infty$ we have

$$
|f(z)| \leq O(\epsilon)+|z|
$$

if $|z| \leq 2$. The same estimate applied to the inverse of $f$ gives $\geq|z|-O(\epsilon)$, so we deduce $|f(z)-z| \leq O(\epsilon)$ on the closed unit disk. The Cauchy estimates then imply $\left|f^{\prime}(z)-1\right|=O(\epsilon /(1-|z|))$, which implies the lemma.

The following lemma from [14] is used in this paper to show that certain boundaries can be "flattened" with small quasiconformal distortion.

Lemma 47 Suppose $G:(x, y) \rightarrow(x, g(x, y))$ is differentiable. Let

$$
\begin{aligned}
& L_{1}(G, z)=\liminf _{w \rightarrow z} \frac{|G(z)-G(w)|}{|z-w|}, \\
& L_{2}(G, z)=\limsup _{w \rightarrow z} \frac{|G(z)-G(w)|}{|z-w|} .
\end{aligned}
$$

Then

$$
\frac{1}{2}(\sqrt{Y}-\sqrt{X})=L_{1}(G, z) \leq L_{2}(G, z)=\frac{1}{2}(\sqrt{Y}+\sqrt{X}),
$$

where

$$
X=1+\left(g_{x}\right)^{2}+\left(g_{y}\right)^{2}-2\left|g_{y}\right|, \quad Y=1+\left(g_{x}\right)^{2}+\left(g_{y}\right)^{2}+2\left|g_{y}\right| .
$$

Thus the quasiconformal dilatation of $G$ is

$$
K(z)=\limsup _{r \rightarrow 0} \frac{\max _{|z-w|=r}|G(z)-G(w)|}{\min _{|z-w|=r}|G(z)-G(w)|}=\frac{(\sqrt{Y}+\sqrt{X})}{(\sqrt{Y}-\sqrt{X})},
$$

and $\mu=\frac{K-1}{K+1}=\sqrt{X} / \sqrt{Y}$. For a map of the form $(x, y) \rightarrow(x+y+g(x, y))$, this becomes $\mu=\sqrt{g_{x}^{2}+g_{y}^{2}} / \sqrt{g_{x}^{2}+\left(2+g_{y}\right)^{2}}$.

The following, which we also use, follows immediately from the above.

Lemma 48 Suppose $W \subset \mathbb{D}$ is a domain defined by $\left\{z=r e^{i \theta}: r<g(\theta)\right\}$, where $1-\delta \leq g \leq 1$ and $\left|g^{\prime}\right| \leq \delta$ for all $\theta$. Then the map $z \rightarrow z / g(\arg (z))$ is $a(1+O(\delta))$ quasiconformal map of $W$ to the disk.

As noted earlier, any quasiconformal map has a dilatation $\mu$ so that $\|\mu\|_{\infty}<1$. Conversely, the "measurable Riemann Mapping theorem" says that given any such $\mu$, there is a $K$-quasiconformal map $f$ with $\mu=\bar{\partial} f / \partial f$. The Beltrami equation $\bar{\partial} f=$ $\mu \partial f$ can be solved using a power series in $\mu$ by setting

$$
f=P[\mu(h+1)]+z,
$$


and

$$
h=T \mu+T \mu T \mu+T \mu T \mu T \mu+\cdots,
$$

where $T$ is the Beurling transform

$$
T h(w)=\lim _{r \rightarrow 0} \frac{1}{\pi} \iint_{|z-w|>r} \frac{h(z)}{(z-w)^{2}} d x d y,
$$

and $P$ is the Cauchy integral

$$
P h(w)=-\frac{1}{\pi} \iint h(z)\left(\frac{1}{z-w}-\frac{1}{z}\right) d x d y .
$$

Formally, $\bar{\partial} P$ is the identity and $\partial P=T$. So if we choose $f$ as above, then

$$
\begin{gathered}
\bar{\partial} f=\mu(h+1), \\
\partial f=T \mu(h+1)+1=h+1 .
\end{gathered}
$$

Hence, $\bar{\partial} f / \partial f=\mu(1+h) /(1+h)=\mu$, as desired. To make the argument rigorous requires $L^{p}$ estimates on these operators as described in [2, Chap. V].

Even though they don't have to be differentiable everywhere, Mori's theorem states that every $K$-quasiconformal map is Hölder continuous of order $1 / K$, i.e.,

$$
|f(x)-f(y)| \leq C|x-y|^{1 / K} .
$$

Moreover, any quasiconformal map of $\mathbb{D}$ to itself extends continuously to the boundary. We shall discuss these boundary values in more detail below.

Numerical computation of quasiconformal maps with given dilatation is considered in [42-44].

\section{A.7 Quasi-isometries}

Quasiconformal maps are a generalization of biLipschitz maps, i.e., maps that satisfy

$$
\frac{1}{K} \leq \frac{|f(x)-f(y)|}{|x-y|} \leq K .
$$

From the metric definition, it is clear that any $K$-biLipschitz map is $K^{2}$-quasiconformal.

For $K$-quasiconformal self-maps of the disk, there is almost a converse. Although a quasiconformal map $f: \mathbb{D} \rightarrow \mathbb{D}$ need not be biLipschitz, it is a quasi-isometry of the disk with its hyperbolic metric $\rho$, i.e., there are constants $A, B$ such that

$$
\frac{1}{A} \rho(x, y)-B \leq \rho(f(x), f(y)) \leq A \rho(x, y)+B .
$$

This says $f$ is biLipschitz for the hyperbolic metric at large scales. A quasi-isometry is also called a rough isometry in some sources, e.g., [79, 133]. We will say $f$ is a quasi-isometry with constant $\epsilon$ if we can take $A=1+\epsilon$ and $B=\epsilon$. 
In [53], Epstein, Marden and Markovic show that any $K$-quasiconformal self-map of the disk is a quasi-isometry with respect to the hyperbolic metric with $A=K$ and $B=K \log 2$ if $1 \leq K \leq 2$ and $B=2.37(K-1)$ if $K>2$. Note that small circles are asymptotically the same for the two metrics, so there is no difference between "hyperbolic-quasiconformal" and "Euclidean-quasiconformal" maps. There is a difference, however, between "hyperbolic biLipschitz" and "Euclidean biLipschitz".

Theorem 49 For a map $f: \mathbb{D} \rightarrow \mathbb{D}$ we have $(1) \Rightarrow(2) \Rightarrow(3) \Rightarrow$ (4) where

(1) $f$ is biLipschitz with respect to the hyperbolic metric.

(2) $f$ is quasiconformal.

(3) $f$ is a quasi-isometry with respect to the hyperbolic metric.

(4) There is a hyperbolic biLipschitz map $g: \mathbb{D} \rightarrow \mathbb{D}$ so that $\left.g\right|_{\mathbb{T}}=\left.f\right|_{\mathbb{T}}$.

In other words, the three classes of maps (hyperbolic biLipschitz, quasiconformal, hyperbolic quasi-isometry) all have the same set of boundary values.

The boundary extension is a quasisymmetric homeomorphism, i.e., there is an $k<\infty$ (depending only on $K$ ) so that $1 / k \leq|f(I)| /|f(J)| \leq k$, whenever $I, J \subset \mathbb{T}$ are adjacent intervals of equal length. Conversely, any quasisymmetric homeomorphism of $\mathbb{T}$ can be extended to a $K$-quasiconformal self-map of the disk, where $K$ depends only on $k$.

\section{A.8 Trees-of-Intervals}

The dyadic intervals of generation $n$ in $\mathbb{R}$ are of the form $\left[2^{-n} j, 2^{-n}(j+1)\right)$ and form the vertices of a infinite binary tree (we say $I, J$ are adjacent if their lengths differ by a factor of 2 and one contains the other). This property is very useful and in this paper we make use of it through the following results.

Lemma 50 Suppose $\mathcal{D}$ is a disjoint, finite collection of dyadic intervals, ordered from left to right, and all with length $\leq L$. Let $\mathcal{C}$ be the collection of all dyadic intervals of length $\leq L$ which contain some element of $\mathcal{D}$. If $\mathcal{C}$ has $m$ elements, it can be enumerated in time $O(m)$.

Proof Start with the leftmost element $I_{1}$ of $\mathcal{D}$ and form the list $\gamma_{1}$, of nested increasing intervals until reaching size $L$. Then move the second element $I_{2}$ of $\mathcal{D}$, and form the nested, increasing list until we hit an element of $\gamma_{1}$. Form $\gamma_{2}$ by cutting $\gamma_{1}$ at this point and replacing the bottom potion by the list starting at $I_{2}$. Continue moving to the right. When we have finished, all $m$ elements of $\mathcal{C}$ has been found and only $O(m)$ work has been done.

More generally, we define a tree-of-intervals as a collection of half-open intervals $\mathcal{I}$ which contains a maximal element (an interval containing all the others) and say $I$ is a child of $J$ if $I \subset J$ and $I$ is maximal in $\mathcal{I}$ with this property. The tree has 
degree $d$ if every interval has at most $d$ children. We will say such a tree is complete if each interval is either a leaf of the tree or is the union of its children.

Lemma 51 Suppose $\mathcal{I}=\left\{I_{j}\right\}_{1}^{n}$ is a tree-of-intervals of degree $d$ and $\mathcal{J}=\left\{J_{j}\right\}_{1}^{m}$ is a complete binary tree-of-intervals. Assume the root of $\mathcal{J}$ contains the root of $\mathcal{I}$. Then for every element of $\mathcal{I}$ we can find the minimum element of $\mathcal{J}$ containing it in total time $O(d \cdot n+m)$.

Proof This is clearly true if either $n=1$ or $m=1$. Consider the case $n>1$ and $m>1$, and suppose the lemma holds for all pairs where both coordinates are strictly smaller. Let $I_{0}, J_{0}$ be the largest elements of $\mathcal{I}$ and $\mathcal{J}$, respectively. Clearly, $I_{0} \subset J_{0}$. Check the children of $J_{0}$ to see if either contains $I_{0}$. Continue this way, creating a path in the $\mathcal{J}$ tree until we either reach (1) a leaf $J$ of $\mathcal{J}$ containing $I_{0}$ or (2) an interval $J$ neither of whose children contain $I_{0}$. This takes work $C_{1} g$ if $J$ is $g$ levels below $J_{0}$, since we only have to do a bounded amount of work per level.

In case (1), we assign $J$ to every element of $\mathcal{I}$ and we are done. In case (2), we assign $J$ to $I_{0}$ and to every descendent of $I_{0}$ which contains the dividing point of $J$ (the common endpoint it's children $J_{1}, J_{2}$ ). These descendent form a path $\gamma$ in the $\mathcal{I}$ tree and if there are $p$ such descendants we can find them all in time $C_{2} d p$ since we only have to check $d$ children at each stage.

Now remove $\gamma$ from the tree $\mathcal{I}$. Each connected component of what remains has its intervals either all in $J_{1}$ or all in $J_{2}$, and thus satisfies the hypothesis of the lemma with respect to the one of the subtrees $\mathcal{J}_{1}, \mathcal{J}_{2}$ of $\mathcal{J}$ rooted at these points. By induction, the assignments can be done for each component $\mathcal{I}_{k}$ in time $C_{3} d \cdot i_{k} \cdot j_{k}$ where $i_{k}$ is the number of vertices in the $\mathcal{I}_{k}$ and $j_{k}$ is the number of vertices in the choice of $\mathcal{J}_{1}$ or $\mathcal{J}_{2}$ covering this component. Summing over all the components gives $C_{3}(d(n-p)+(m-g))$. Added to the $C_{1} g+C_{2} d p$ already done, this proves the lemma.

This seems closely related to merging heaps in computer science (a heap is a tree whose vertices are labeled by numbers so that the children's labels are less than the parents'), although here the labels are intervals and the ordering is set inclusion.

In Sect. 14, following Lemma 29 we claimed that in linear time we could find gaps or crescents that were a bounded hyperbolic distance from the image of each Whitney box. Here we give a few more details. Associate to each Whitney square, $Q_{j}$, its base $I_{j} \subset \mathbb{R}$. Then map $I_{j}$ to $\partial \Omega_{k}$ by the map $f$ and back to $\mathbb{R}$ by $\varphi_{k}$ (the iota map for $\Omega_{k}$ ). Let $W_{j}$ be the image of $Q_{j}$ under these two maps. Since $\varphi_{k}$ is a quasi-isometry, $W_{j}$ is a bounded hyperbolic distance from the Whitney box corresponding to the image $K_{j}$ of $I_{j}$. The $\left\{K_{j}\right\}$ still form a tree-of-intervals which we denote $\mathcal{K}$. Let $\mathcal{J}$ be the complete binary tree-of-intervals consisting of the bases of all the bending lamination geodesics. If $J \in \mathcal{J}$ is the minimal element containing $K \in \mathcal{K}$, then the ideal triangle (or crescent if $J$ is a leaf) bounded above by the geodesic corresponding to $J$ must hit the Whitney box corresponding to $K$ and thus is a bounded hyperbolic distance from $W_{j}$. This is what we wanted. Thus finding the claimed gaps/crescents, reduces to an application of Lemma 51. 


\section{Appendix B: Fast Power Series Manipulations (or, $O(n \log n)$ Suffices)}

In order to prove Theorems 1 or 2 with $C(\epsilon)=O\left(\log \frac{1}{\epsilon} \log \log \frac{1}{\epsilon}\right)$, we can only use operations on power series of length $p \sim \log \frac{1}{\epsilon}$ that take time at most $O(p \log p)$. In this section, we review some basic results about power series manipulations and check that all the operations we need can be carried out this quickly. If one uses naive manipulations of power series, then one simply gets Theorem 2 with a larger constant, e.g., $C(\epsilon)=O\left(\log ^{c} \frac{1}{\epsilon}\right)$ for some $c$. To conform with the references, we replace $p$ by $n$; from this point on, $n$ will refer to the number of terms in a power series (rather than the number of vertices in a polygon, as it did in earlier sections). This summary is taken mostly from $[122,128]$.

The Fourier matrix is given by

$$
F_{n}=\left(\begin{array}{ccccc}
1 & 1 & 1 & \ldots & 1 \\
1 & \omega & \omega^{2} & \ldots & \omega^{n-1} \\
1 & \omega^{2} & \omega^{4} & \ldots & \omega^{2(n-1)} \\
\vdots & \vdots & \vdots & \ddots & \vdots \\
1 & \omega^{n-1} & \omega^{2(n-1)} & \ldots & \omega^{(n-1)(n-2)}
\end{array}\right)
$$

where $\omega$ is an $n$th root of unity. The fast Fourier transform (FFT) applies $F_{n}$ to an $n$-vector in time $O(n \log n)$ [40]. $F_{n}$ is unitary (after rescaling) and its conjugate transpose, $F^{*}$, can also be applied in $O(n \log n)$ time. The discrete Fourier transform (DFT) takes a $n$-long sequence of complex numbers $\left\{a_{k}\right\}_{0}^{n-1}$ and a $n$-root of unity $\omega$ and returns the values of the polynomial $p(z)=a_{0}+a_{1} x+\cdots+a_{n-1} z^{n-1}$ at the points $z=\left\{1, \omega, \omega^{2}, \ldots, \omega^{n-1}\right\}$. Composing DFT with its adjoint returns the original sequence times $n$.

Suppose $f(z)=\sum_{k=0}^{n} a_{k} z^{k}$ and $g(z)=\sum_{k=0}^{n} b_{k} z^{k}$. How fast can we multiply, divide or compose these series? Let $M(n)$ denote the number of field operations it takes to multiply two power series of length $n$. The usual process of convolving the coefficients shows $M(n)=O\left(n^{2}\right)$. A divide and conquer method of Karatsuba and Ofman [85] improves this to $O\left(n^{\alpha}\right)$ with $\alpha=\log 3 / \log 2$, but the fastest known method uses the Fast Fourier Transform [40], which shows $M(n)=O(n \log n)$ (two power series of length $n$ can be multiplied by taking the DFT of each, multiplying the results term-by-term, taking the DFT of the result and finally dividing by $n$ ).

Other operations on power series are generally estimated in terms of $M(n)$. For example, inversion (finding the reciprocal power series, $1 / f$, given the series for $f$ ) is $O(M(n))$. Like several other operations on power series, this is most easily proven using Newton's method (applied to series rather than numbers). For example, $1 / f$ is the solution of the equation $\frac{1}{g}-f=0$. If $g_{k}$ is an approximate solution with $n>0$ terms correct, then

$$
g_{k+1}=g_{k}-\frac{\frac{1}{g_{k}}-f}{-1 / g_{k}^{2}}=g_{k}-\frac{f g_{k}-1}{z^{n}} g_{k} z^{n}
$$

has $2 n$ correct terms. The right side requires two multiplications and so the work to compute inversions is $O(M(n))+O(M(n / 2))+\cdots+O(1)=O(M(n))$. 
Given inversion, one can divide power series (multiply $f$ by $1 / g$ ) compute $\log f$ (integrate $f^{\prime} / f$ term-by-term) or $\exp (f)$ (solve $\log g=f$ by Newton's method) all in time $O(M(n))$.

Composition of power series is a little harder. Brent and Kung showed that given power series $f, g$ of order $n$ and $g_{0}=0$, the composition $f \circ g$ can be computed in time $\operatorname{Comp}(n)=O(\sqrt{n \log n} M(n))$. Using FFT multiplication, this gives $O\left(n^{3 / 2} \log ^{3 / 2} n \log \log n\right)$. They also showed that reversion (i.e., given $f$ find $g$ so $f \circ g(z)=z$ ) can be solved using Newton's method with the iteration

$$
g \rightarrow g-\frac{f \circ g}{f^{\prime} \circ g},
$$

which doubles the number of correct terms in $g$ with every step. Thus $\operatorname{Rev}(n)=$ $O(\operatorname{Comp}(n))=O(\sqrt{n \log n} M(n))$.

Fortunately, there are some special cases when composition is faster. For example, if we want to post-compose with a linear fractional transformation $\sigma(z)=(a z+$ $b) /(c z+d)$, this is the same as adding and dividing series, so is only $O(M(n))$.

Pre-composing by $\sigma$ is more complicated. A function $f$ is called algebraic if it satisfies

$$
P_{d}(z) f(z)^{d}+\cdots+P_{0}(z)=0,
$$

for some polynomials $P_{0}, \ldots, P_{d}$. Clearly, every rational function is algebraic with $d=1$. The power series of algebraic functions satisfy linear recursions and $n$ terms of the series can be computed in $O(n)$. Moreover, pre-composition by algebraic functions is fast; if $f$ has $p$ terms, $g$ has $q$ terms and is algebraic of degree $d$ then the first $n$ terms of $f \circ g$ can be computed in time $O\left(q d^{2} \frac{p(q-v)}{n} M(n+p v) \log n\right)$ where $v$ is the valuation of $P_{d}$ (the largest power of $z$ that divides $\left.P_{d}(z)\right)$ and $q$ is the maximum of the degrees of $P_{i}$, plus 1 . For linear fractional transformations, $v=0$ and $q=2$ so the time to pre-compose by such a map is $O(M(n) \log n)=O\left(n \log ^{2} n\right)$. (There is an extensive generalization of the algebraic case to fast manipulations of holonomic functions, as developed by van der Hoeven [127], although we do not need to use it here.)

This is too slow for our purposes. Fortunately, the only times we will have to precompose with a Möbius transformation correspond to various manipulations of power and Laurent series in the fast multipole method and all of these can be accomplished in $O(n \log n)$ by fast application of Toeplitz, Hankel and Pascal matrices as shown by Tang in [122] (the following discussion is based on [122]).

A matrix is called circulant if each column is a down-shift of the previous one, is called Toeplitz if it is constant on diagonals (slope -1) and called Hankel if it is constant on antidiagonals (slope 1). The general forms of these three types are:

$$
C(x)=\left(\begin{array}{ccccc}
x_{1} & x_{n} & x_{n-1} & \ldots & x_{2} \\
x_{2} & x_{1} & x_{n} & \ldots & x_{3} \\
x_{3} & x_{2} & x_{1} & \ldots & x_{4} \\
\vdots & \vdots & \vdots & \ddots & \vdots \\
x_{n} & x_{n-1} & x_{n-2} & \ldots & x_{1}
\end{array}\right),
$$




$$
\begin{gathered}
T(x)=\left(\begin{array}{ccccc}
x_{0} & x_{1} & x_{2} & \ldots & x_{n-1} \\
x_{-1} & x_{0} & x_{1} & \ldots & x_{n-2} \\
x_{-2} & x_{-1} & x_{0} & \ldots & x_{n-3} \\
\vdots & \vdots & \vdots & \ddots & \vdots \\
x_{-n+1} & x_{-n+2} & x_{-n+3} & \ldots & x_{0}
\end{array}\right), \\
H(x)=\left(\begin{array}{ccccc}
x_{-n+1} & x_{-n+2} & x_{-n+3} & \ldots & x_{0} \\
x_{-n+2} & x_{-n+3} & x_{-n+4} & \ldots & x_{1} \\
x_{-n+3} & x_{-n+4} & x_{-n+5} & \ldots & x_{2} \\
\vdots & \vdots & \vdots & \ddots & \vdots \\
x_{0} & x_{1} & x_{2} & \ldots & x_{n-1}
\end{array}\right) .
\end{gathered}
$$

A circulant matrix can be applied to a vector using three applications of FFT, i.e., because $C_{n}(x)$ applied to a vector $y$ is the same as $\operatorname{IFFT}(\operatorname{FFT}(x) \cdot \operatorname{FFT}(y))$. A Toeplitz matrix can be embedded in a circulant matrix of the form

$$
C_{2 n}=\left(\begin{array}{ll}
T_{n} & S_{n} \\
S_{n} & T_{n}
\end{array}\right)
$$

where

$$
S_{n}=\left(\begin{array}{ccccc}
0 & x_{-n+1} & x_{-n+2} & \ldots & x_{-1} \\
x_{n-1} & 0 & x_{-n+1} & \ldots & x_{-2} \\
x_{n-2} & & 0 & \ldots & x_{-3} \\
\vdots & \vdots & \vdots & \ddots & \vdots \\
x_{1} & x_{2} & x_{3} & \ldots & 0
\end{array}\right)
$$

To apply $T$ to an $n$-vector $y$, append $n$ zeros to $y$ to get a $2 n$-vector, apply $C_{n}$ and take the first $n$ coordinates of the result. This takes $O(n \log n)$ time. If $H$ is a Hankel matrix then $R \cdot H$ is a Toeplitz matrix where $R$ is the permutation matrix, that is, $1 \mathrm{~s}$ on the main anti-diagonal and 0 s elsewhere, i.e., it reverses the order of the coordinates of a vector. Thus $H=R \cdot(R \cdot H)$, is a Toeplitz matrix followed by a permutation and can clearly be applied in time $O(n \log n)$ as well.

The Pascal matrix is lower triangular with its $(j, k)$ th entry being the binomial coefficient $C_{i}^{j}=\left(\begin{array}{c}i \\ j\end{array}\right)$ :

$$
\left(\begin{array}{ccccc}
1 & 0 & 0 & \ldots & 0 \\
1 & 1 & 0 & \ldots & 0 \\
1 & 2 & 1 & \ldots & 0 \\
\vdots & \vdots & \vdots & \ddots & \vdots \\
C_{n-1}^{0} & C_{n-1}^{1} & C_{n-1}^{2} & \ldots & C_{n-1}^{n-1}
\end{array}\right)
$$

This matrix can be written as $P=\operatorname{diag}\left(v_{1}\right) \cdot T \cdot \operatorname{diag}\left(v_{2}\right)$ where

$$
v_{1}=(1,1,2 !, 3 !, \ldots,(n-1) !),
$$


$v_{2}=\frac{1}{v_{1}}$ (term-wise) and $T$ is the Toeplitz matrix

$$
T=\left(\begin{array}{ccccc}
1 & 0 & 0 & \ldots & 0 \\
1 & 1 & 0 & \ldots & 0 \\
\frac{1}{2 !} & 1 & 1 & \ldots & 0 \\
\vdots & \vdots & \vdots & \ddots & \vdots \\
\frac{1}{(n-1) !} & \frac{1}{(n-2) !} & \frac{1}{(n-3) !} & \ldots & 1
\end{array}\right)
$$

The diagonal matrices can be applied in $O(n)$ and the Toeplitz in $O(n \log n)$ and hence so can $P$. Similarly for the transpose of $P$.

Now for the applications to fast multipole translation operators. There are three types of conversions to consider. First, local-to-local translation

$$
\sum_{k=0}^{n-1} a_{k}(z-a)^{k} \rightarrow \sum_{k=0}^{n-1} b_{k}(z-b)^{k}
$$

then multipole-to-local

$$
\sum_{k=0}^{n} a_{k}(z-a)^{-k} \rightarrow \sum_{k=0}^{n} b_{k}(z-b)^{k}
$$

and finally, multipole-to-multipole,

$$
\sum_{k=0}^{n} a_{k}(z-a)^{-k} \rightarrow \sum_{k=0}^{n} b_{k}(z-b)^{-k} .
$$

Let $c=b-a$ and consider the local-to-local translation. We have

$$
\sum_{k=0}^{n-1} a_{k}(w-c)^{k}=\sum_{k=0}^{n-1} a_{k} \sum_{j=0}^{k} w^{j}(-c)^{k-j}\left(\begin{array}{l}
k \\
j
\end{array}\right),
$$

so the matrix corresponding to local translation has $k$ th column

$$
\left((-c)^{k},(-c)^{k-1}\left(\begin{array}{l}
k \\
1
\end{array}\right), \ldots,(-c)^{0}\left(\begin{array}{l}
k \\
k
\end{array}\right), 0, \ldots, 0\right)^{t}
$$

or

$$
L L=\left(\begin{array}{ccccc}
1 & -c & c^{2} & \ldots & (-c)^{n-1} \\
0 & 1 & -2 c & \ldots & (-c)^{n-2} C_{n-1}^{1} \\
0 & 0 & 1 & \ldots & (-c)^{n-3} C_{n-1}^{2} \\
\vdots & \vdots & \vdots & \ddots & \vdots \\
0 & 0 & 0 & \ldots & 1
\end{array}\right)
$$


This matrix is equal to

$$
\operatorname{diag}\left(1,-c, \ldots,(-c)^{n-1}\right) \cdot P^{\prime} \cdot \operatorname{diag}\left(1,-c^{-1}, \ldots,(-c)^{-n+1}\right),
$$

where $P^{\prime}$ is the transpose of $P$. The diagonal matrices can be applied in $O(n)$ time and $P^{\prime}$ can be applied in $O(n \log n)$. Thus local-to-local translations can be done this fast.

Similarly, the multipole-to-multipole and multipole-to-local transformations correspond to applying the matrices

$$
\begin{aligned}
& M M=\left(\begin{array}{ccccc}
1 & 0 & 0 & \ldots & 0 \\
\left(\begin{array}{l}
1 \\
1
\end{array}\right) c & 1 & 0 & \ldots & 0 \\
\left(\begin{array}{l}
2 \\
2
\end{array}\right) c^{2} & \left(\begin{array}{l}
2 \\
1
\end{array}\right) c & 1 & \ldots & \\
\vdots & \vdots & \vdots & \ddots & \vdots \\
\left(\begin{array}{c}
n-1 \\
n-1
\end{array}\right) c^{n-1} & \left(\begin{array}{c}
n-1 \\
n-2
\end{array}\right) c^{n-2} & \left(\begin{array}{c}
n-1 \\
n-3
\end{array}\right) c^{n-3} & \ldots & 1
\end{array}\right), \\
& M L=\left(\begin{array}{ccccc}
-c^{-1} & c^{-2} & c^{-3} & \ldots & c^{-n+1} \\
-c^{-2} & 2 c^{-3} & -3 c^{-4} & \ldots & \\
-c^{-3} & 3 c^{-4} & -6 c^{-5} & \ldots & \\
\vdots & \vdots & \vdots & \ddots & \vdots \\
-c^{-n+1} & (n-1) c^{-n} & -\left(\begin{array}{c}
n \\
2
\end{array}\right) c^{-n-1} & \ldots & -1^{n-1}(\underset{c}{2 p-2, p-1})^{-2 n-1}
\end{array}\right)
\end{aligned}
$$

We can rewrite these matrices as

$$
\begin{gathered}
M M=\operatorname{diag}\left(1, c, \ldots, c^{n-1}\right) \cdot P \cdot \operatorname{diag}\left(1, c^{-1}, \ldots, c^{-n+1}\right), \\
M L=\operatorname{diag}\left(1, c^{-1}, \ldots, c^{1-n}\right) \cdot P \cdot P^{\prime} \cdot \operatorname{diag}\left(-c^{-1}, c^{-2} \ldots(-c)^{-n}\right),
\end{gathered}
$$

where $P^{\prime}$ is the transpose of $P$. As with local translations, these are compositions of diagonal matrices (which can be applied in $O(n)$ ) and matrices that can be applied in $O(n \log n)$ time.

We will also use structured matrices to compute expansions around $\infty$ of functions of the form $\int \frac{d \mu(z)}{(z-w)^{k}}, k=1,2,3$. We will only consider the Cauchy transform $(k=1)$ since the others can be obtained by term-by-term differentiation of that one. Suppose $f(z)=\sum_{k=0}^{n} a_{k} z^{k}$ is a power series for an analytic function, bounded by one and defined on $\mathbb{D}$ and $\varphi(x, y)$ is a polynomial in $x$ and $y$ of uniformly bounded degree. Then the Cauchy transform

$$
F(w)=\int_{S} \frac{f(z) \varphi(x, y) d x d y}{z-w},
$$

is analytic in $w$ outside $S=\left[-\frac{1}{2}, \frac{1}{2}\right]^{2}$, so has an expansion $F(w)=\sum_{k=1}^{\infty} b_{n} w^{-n}$. Given $\left\{a_{k}\right\}_{0}^{n}$, thinking of $\varphi$ as fixed, we want to compute $\left\{b_{k}\right\}_{1}^{n}$. For each monomial of the form $z^{k} x^{a} y^{b}$, we can precompute the expansion using explicit formulas $(O(n)$ for each of $O(n)$ monomials) and then we simply apply the resulting matrix to the vector $\left\{a_{k}\right\}$. Naively, we can do this in time $O\left(n^{2}\right)$. 
Actually, we can compute the expansion in only $O(n \log n)$. Let $d \mu=x^{a} y^{b} d x d y$ restricted to $Q=[0,1]^{2}$. We want to compute the expansion at $\infty$ of

$$
\begin{aligned}
F(w)=\iint \frac{z^{n}}{w-z} d \mu(z) & =\iint z^{n} \frac{1}{w}\left(1+\frac{z}{w}+\left(\frac{z}{w}\right)^{2}+\cdots\right) d \mu(z) \\
& =\sum_{k=0}^{\infty} w^{-k-1} \iint z^{n+k} d \mu(z) \\
& =\sum_{k=1}^{\infty} a_{k, n} w^{-k},
\end{aligned}
$$

where

$$
a_{k, n}=c(n+k+1, a, b)=\iint_{Q}(x+i y)^{n+k-1} x^{a} y^{b} d x d y .
$$

Since $a_{k, n}$ only depends on $k+n, A$ is a Hankel matrix. As noted above, a $n \times n$ Hankel matrix can be applied to a $n$-vector using FFT in time $O(n \log n)$.

The individual coefficients have explicit formulas involving Euler's Beta function. Evaluations for a few small values of $a, b$ (as given by Mathematica are)

$$
\begin{gathered}
c(n, 0,0)=\frac{i-i^{n+1}+2(1+i)^{n}}{2+3 n+n^{2}}, \\
c(n, 1,0)=\frac{2 i-i^{n}+i n+2(1+i)^{n}((2-i)+n)}{(1+n)(2+n)(3+n)}, \\
c(n, 2,0)=\frac{i\left(6+2 i^{n}+5 n+n^{2}\right)+2(1+i)^{n}((4-4 i)+n((5-2 i)+n))}{(1+n)(2+n)(3+n)(4+n)}, \\
c(n, 1,1)=-\frac{1+i^{n}-2(1+i)^{n}(2+n)}{(1+n)(2+n)(4+n)}, \\
c(n, 2,1)=\frac{-3(2 i) i^{n}-n+2(1+i)^{n}(1+n)((4-i)+n)}{(1+n)(2+n)(3+n)(5+n)}, \\
c(n, 0,1)=\frac{-1-i^{n+1}(2+n)+2(1+i)^{n}((2+i)+n)}{(1+n)(2+n)(3+n)} .
\end{gathered}
$$

Thus $n$-term Laurent expansions for Beurling transforms of the appropriate degree $n$ polynomials can be computed in time $O(n \log n)$.

In Sect. 11, we claimed that $\epsilon$-representations and partial representations could be computed from each other quickly. We can now see why this is true. Given a $O(n)$ term Laurent expansion on an annulus we can clearly create $O(n)$-term power series expansions that approximate it on disks contained in the annulus in time $O(n \log n)$, just as with the multipole to local conversions discussed before. If the double of the disk is contained in the annulus where the function is bounded by 1 , then the convergence of the power series is geometric and $O(n)$ terms give accuracy of order 
$\lambda^{n}$ for some $\lambda<1$. Thus we can do the conversion from representations to partial representations.

To go the other direction, we need to compute the coefficients of a Laurent expansion for $f$ from knowing power series approximations for $f$ on disks that cover a contour $\gamma$ around the origin. The coefficients can be computed exactly as integrals of the form $\int_{\gamma} f(z) z^{k} d z$. This integral can be broken into pieces $\int_{\gamma_{n}}$ where $\gamma=\bigcup_{n} \gamma_{n}$ is a decomposition of $\gamma$ into pieces, where each piece stays inside one of the disks where we have a power series approximation for $f$. If this approximation is of the form $\sum_{j} a_{j}(z-a)^{j}$, then we wish to evaluate integrals of the form

$$
\int_{\gamma_{n}}\left(\sum_{j} a_{j}(z-a)^{j}\right) z^{k} d z .
$$

We can convert $z^{k}$ to a series with center $a$, e.g., $z^{k}=\sum_{q} b_{q, k}(z-a)^{q}$ just as before with Pascal matrices and then we have to evaluate

$$
\begin{aligned}
\int_{\gamma_{n}}\left(\sum_{j} a_{j}(z-a)^{j}\right)\left(\sum_{q} b_{q, k}(z-a)^{q}\right) d z & =\sum_{q} \sum_{j} a_{k} b_{q, k} \int_{\gamma_{n}}(z-a)^{j+q} d z \\
& =\sum_{q} I_{j, k} \sum_{j} b_{q, k} a_{k},
\end{aligned}
$$

where $b_{q, k}$ is a Pascal matrix and $I_{j, q}=\int_{\gamma_{n}}(z-a)^{j+q} d z$ is an explicit Hankel matrix. We have seen above that both types of matrix can be applied in $O(n \log n)$. So that the contour integral using the power series on each piece of the contour can be evaluated this fast. If the power series approximations agree with the Laurent series to within $\epsilon$ then resulting coefficients will be accurate up to an error of $\int_{\gamma} \epsilon|d z|=O(|\gamma| \epsilon)$. Normalizing so that $\gamma$ and its image both have length about 1, we see that the coefficients are accurate to $O(\epsilon)$ and so the reconstructed Laurent series agrees with the original to within $\sum_{k} \epsilon=O(n \epsilon)=O(\epsilon \log \epsilon)=O\left(\epsilon^{1-\beta}\right)$ with $\beta$ as close to 0 as we wish (taking a larger constant in front).

The compositions of power series considered earlier were exact computations in the sense that given two $n$-term power series, $f$ and $g$, we are computing the exact first $n$ coefficients of $f \circ g$. However, they are inexact in the sense that we are truncating up to $n^{2}-n$ terms of the full composition. We will be most interested in the case when $f$ and $g$ are conformal maps whose power series coefficients decay exponentially. Then the $n$-term truncation of $f \circ g$ equals $f \circ g$ up to an error of $e^{-c n}$. Since the truncation introduces an error anyway, we may as well tolerate an error of the same size coming from an inexact computation of the first $n$ terms that we keep. Can we approximate the first $n$ terms of $f \circ g$ faster than we can compute them exactly (i.e., faster than $\left.O\left(n^{3 / 2} \log ^{3 / 2} n \log \log n\right)\right)$ ? The answer is yes, at least in the special case that we care about.

Lemma 52 Suppose $f$ and $g$ are conformal maps of $D(0, R), R \geq 2$ such that $f$ has $a(1+\epsilon)$-quasiconformal extension to $\mathbb{R}^{2}$ that fixes $\infty$. Assume also that $f(0)=g(0)$ and $f^{\prime}(0)=g^{\prime}(0)=1$. Then given the first $n$ terms of the power series for $f$ and $g$ we can compute in time $O(n \log n)$, an $O(n)$ term power series $h$ so that $\left|h-g \circ f^{-1}\right| \leq$ $O\left(\epsilon^{2(1-\beta)}\right)$ on $D(0,1)$. 
Proof Let $\left\{\omega_{j}\right\}_{0}^{n-1}$ denote the $n$th roots of unity. Using the FFT we can compute the images of all these points under either $f$ or $g$ in time $O(n \log n)$. By Lemma 46, $\left|f^{\prime}-1\right|=O(\epsilon)$ on $D(0,2)$ and hence if $\left|z-\omega_{j}\right|=O(\epsilon)$,

$$
f(z)=f\left(\omega_{j}\right)+(1+O(\epsilon))\left(z-\omega_{j}\right)+O\left(\left|z-\omega_{j}\right|^{2}\right) .
$$

In particular, if $w=f(z)$,

$$
\begin{aligned}
f^{-1}(w) & =w+\left(\omega_{j}-f\left(\omega_{j}\right)\right)+O\left(\epsilon\left|z-\omega_{j}\right|\right)+O\left(\left|z-\omega_{j}\right|^{2}\right) \\
& =w+\left(\omega_{j}-f\left(\omega_{j}\right)\right)+O\left(\epsilon^{2}\right) .
\end{aligned}
$$

Thus taking $w=\omega_{j}$,

$$
f^{-1}\left(\omega_{j}\right)=\omega_{j}+\left(\omega_{j}-f\left(\omega_{j}\right)\right)+O\left(\epsilon^{2}\right) .
$$

Therefore, we get

$$
\begin{aligned}
g \circ f^{-1}\left(\omega_{j}\right) & =g\left(\omega_{j}\right)+g^{\prime}\left(\omega_{j}\right)\left(\omega_{j}-f\left(\omega_{j}\right)\right)+O\left(\left|\omega_{j}-f\left(\omega_{j}\right)\right|^{2}\right)+O\left(\epsilon^{2}\right) \\
& =g\left(\omega_{j}\right)+g^{\prime}\left(\omega_{j}\right)\left(\omega_{j}-f\left(\omega_{j}\right)\right)+O\left(\epsilon^{2}\right) .
\end{aligned}
$$

So define a degree $n$ polynomial by setting its values on the roots of unity using

$$
h\left(\omega_{j}\right)=g\left(\omega_{j}\right)+g^{\prime}\left(\omega_{j}\right)\left(\omega_{j}-f\left(\omega_{j}\right)\right) .
$$

Since we can compute and evaluate $g$ and $g^{\prime}$ at the roots of unity in $O(n \log n)$, we can find the series expansion for $h$ around 0 in time $O(n \log n)$ using an FFT.

Clearly, $h$ is a good approximation to $g \circ f^{-1}$ on the $n$th roots of unity (they differ by $O\left(\epsilon^{2}\right)$ on these points). What about the rest of $\mathbb{D}$ ? We shall show that the functions differ by at most $O\left(\epsilon^{2}\right)$ on $\mathbb{D}$ as well. Let

$$
\sigma(z)=\prod_{j=0}^{n-1}\left(z-\omega_{j}\right)=z^{n}-1
$$

and

$$
\psi(z)=\sum_{k=0}^{n-1} \frac{h\left(\omega_{j}\right) /\left(n \omega_{j}^{n-1}\right)}{z-\omega_{j}} .
$$

The zeros of $\sigma$ cancel the simple poles of $\psi$, so $P=\psi \cdot \sigma$ is entire and since $|\sigma| \sim$ $|z|^{n}$ and $|\psi| \sim|z|^{-1}$ near $\infty, P$ must be a polynomial of degree $n-1$. Moreover, by l'Hôpital's rule,

$$
\begin{aligned}
\frac{h\left(\omega_{j}\right)}{n \omega_{j}^{n-1}} & =\lim _{z \rightarrow \omega_{j}}\left(z-\omega_{j}\right) \psi(z) \\
& =\lim _{z \rightarrow \omega_{j}}\left(z-\omega_{j}\right) \frac{P(z)}{\sigma(z)} \\
& =\frac{1 \cdot P\left(\omega_{j}\right)+\left(\omega_{j}-\omega_{j}\right) P^{\prime}\left(\omega_{j}\right)}{\sigma^{\prime}\left(\omega_{j}\right)}=\frac{P\left(\omega_{j}\right)}{\sigma^{\prime}\left(\omega_{j}\right)},
\end{aligned}
$$


which implies $P\left(\omega_{j}\right)=h\left(\omega_{j}\right)$ for all $j=0, \ldots, n-1$, and this means $P=h$ as polynomials. Thus

$$
\begin{aligned}
|h(z)| & =|P(z)| \leq|\psi(z)| \cdot|\sigma(z)| \\
& \leq\left(\max _{j}\left|h\left(\omega_{j}\right)\right|\right) \sum_{j=0}^{n-1} \frac{1 / n}{\left|z-\omega_{j}\right|} \cdot\left(\left|z^{n}\right|+1\right) \\
& \leq 4 M,
\end{aligned}
$$

if $|z| \leq 1 / 2$ and $M=\max _{j}\left|h\left(\omega_{j}\right)\right|$.

Suppose $1<r<R / 2$. The map $g \circ f^{-1}$ is conformal on $D(0, R / 2)$ (if $\epsilon$ is small enough), so its power series at 0 has terms that decay like $(2 / R)^{k}$. If $H$ is the $m$ term truncation of this power series and we choose $m \sim n$ large enough, then $\mid H-$ $g \circ f^{-1} \mid \leq O\left((2 / R)^{m}\right)=O\left(\epsilon^{2}\right)$ on $\mathbb{D}$. Since $H$ is a degree $n-1$ polynomial that interpolates its own values on the roots of unity, $H-h$ is a degree $n-1$ polynomial with values $=O\left((2 / R)^{n}\right)$ on the $n$th roots of unity and hence, by our calculation above applied to $H-h$, is bounded by $O\left(\epsilon^{2}\right)$ on $\mathbb{D}$. By Lemma 21 , this means that a truncation $h_{t}$ of $h$ satisfies

$$
\left|h_{t}(z)-g \circ f^{-1}(z)\right|=O\left(\epsilon^{2(1-\beta)}\right),
$$

on $D(0, r)$ where $r=R^{\beta}$.

Exact evaluation of a degree $n$ polynomial at $m$ points takes $O((n+m)$ $\left.\log ^{2}(n+m)\right)$ [3] and recovering a degree $n-1$ polynomial from its values at $n$ points takes $O\left(n \log ^{2} n\right)$, so our approximate method is faster for the cases we consider. Multipole methods can also be used to give faster approximate evaluation and interpolation algorithms; see [51, 107]. These are $O\left(n \log \frac{1}{\epsilon}\right)$ where $n$ is the degree and $\epsilon$ is the desired accuracy. In our case, however, $n \sim \log \frac{1}{\epsilon}$, so this is not faster than exact calculation.

\section{References}

1. Aggarwal, A., Guibas, L.J., Saxe, J., Shor, P.W.: A linear-time algorithm for computing the Voronor diagram of a convex polygon. Discrete Comput. Geom. 4(6), 591-604 (1989)

2. Ahlfors, L.V.: Lectures on Quasiconformal Mappings. The Wadsworth \& Brooks/Cole Mathematics Series. Wadsworth \& Brooks/Cole Advanced Books \& Software, Monterey (1987). With the assistance of Clifford J. Earle, Jr., reprint of the 1966 original

3. Aho, A.V., Steiglitz, K., Ullman, J.D.: Evaluating polynomials at fixed sets of points. SIAM J. Comput. 4(4), 533-539 (1975)

4. Aurenhammer, F.: Voronoi diagrams-a survey of a fundamental geometric data structure. ACM Comput. Surv. 23, 345-405 (1991)

5. Aurenhammer, F., Klein, R.: Voronoi Diagrams. In: Handbook of Computational Geometry, pp. 201-290. North-Holland, Amsterdam (2000)

6. Banchoff, T.F., Giblin, P.J.: Global theorems for symmetry sets of smooth curves and polygons in the plane. Proc. R. Soc. Edinb. A 106(3-4), 221-231 (1987)

7. Banjai, L., Trefethen, L.N.: A multipole method for Schwarz-Christoffel mapping of polygons with thousands of sides. SIAM J. Sci. Comput. 25(3), 1042-1065 (2003) (electronic) 
8. Bern, M., Eppstein, D.: Mesh generation and optimal triangulation. In: Computing in Euclidean Geometry. Lecture Notes Ser. Comput, vol. 1, pp. 23-90. World Scientific, River Edge (1992)

9. Beurling, A., Ahlfors, L.: The boundary correspondence under quasiconformal mappings. Acta Math. 96, 125-142 (1956)

10. Binder, I., Braverman, M., Yampolsky, M.: On the computational complexity of the Riemann mapping. Ark. Mat. 45(2), 221-239 (2007)

11. Bishop, C.J.: Divergence groups have the Bowen property. Ann. Math. (2) 154(1), 205-217 (2001)

12. Bishop, C.J.: BiLipschitz approximations of quasiconformal maps. Ann. Acad. Sci. Fenn. Math. 27(1), 97-108 (2002)

13. Bishop, C.J.: Quasiconformal Lipschitz maps, Sullivan's convex hull theorem and Brennan's conjecture. Ark. Mat. 40(1), 1-26 (2002)

14. Bishop, C.J.: An explicit constant for Sullivan's convex hull theorem. In: In the Tradition of Ahlfors and Bers, III. Contemp. Math., vol. 355, pp. 41-69. Am. Math. Soc., Providence (2004)

15. Bishop, C.J., Hakobyan, H.: A central set of dimension 2. Proc. Am. Math. Soc. 136(7), 2453-2461 (2008)

16. Bishop, C.J.: Bounds for the CRDT conformal mapping algorithm. Comput. Methods Funct. Theory 10(1), 325-366 (2010)

17. Bishop, C.J.: A fast QC-mapping theorem for polygons. Preprint (2009)

18. Bishop, E., Bridges, D.: Constructive Analysis. Grundlehren der Mathematischen Wissenschaften [Fundamental Principles of Mathematical Sciences], vol. 279. Springer, Berlin (1985)

19. Blum, H.: A transformation for extracting new descriptors of shape. In: Dunn, W.W. (ed.) Proc. Sympos. Models for the Perception of Speech and Visual Form, pp. 362-380. MIT Press, Cambridge (1967)

20. Blum, H.: Biological shape and visual science. J. Theoret. Biol. 38, 205-287 (1973)

21. Blum, H., Nagel, R.N.: Shape descriptors using weighted symmetric axis functions. Pattern Recogn. 10(3), 167-180 (1978)

22. Bridgeman, M.: Average bending of convex pleated planes in hyperbolic three-space. Invent. Math. 132(2), 381-391 (1998)

23. Bridgeman, M.: Average curvature of convex curves in $H^{2}$. Proc. Am. Math. Soc. 126(1), 221-224 (1998)

24. Bridgeman, M., Canary, R.D.: From the boundary of the convex core to the conformal boundary. Geom. Dedicata 96, 211-240 (2003)

25. Bruce, J.W., Giblin, P.J., Gibson, C.G.: Symmetry sets. Proc. R. Soc. Edinb. A 101(1-2), 163-186 (1985)

26. Canary, R.D.: The conformal boundary and the boundary of the convex core. Duke Math. J. 106(1), 193-207 (2001)

27. Carleson, L.: Interpolations by bounded analytic functions and the corona problem. Ann. Math. (2) 76, 547-559 (1962)

28. Chazai, F., Lieutier, A.: Stability and homotopy of a subset of the medial axis. In: Proc. 9th ACM Sympos. Solid Modeling Appl. (2004)

29. Chazal, F., Soufflet, R.: Stability and finiteness properties of medial axis and skeleton. J. Dynam. Control Syst. 10(2), 149-170 (2004)

30. Chazelle, B.: Triangulating a simple polygon in linear time. Discrete Comput. Geom. 6(5), 485-524 (1991)

31. Cheng, H.: A constructive Riemann mapping theorem. Pac. J. Math. 44, 435-454 (1973)

32. Chiang, C.-S., Hoffmann, C.M.: The medial axis transform for 2 d regions. ACM Transactions on graphics (1982)

33. Chin, F., Snoeyink, J., Wang, C.A.: Finding the medial axis of a simple polygon in linear time. Discrete Comput. Geom. 21(3), 405-420 (1999)

34. Choi, H.I., Choi, S.W., Moon, H.P.: Mathematical theory of medial axis transform. Pac. J. Math. 181(1), 57-88 (1997)

35. Choi, S.W., Seidel, H.-P.: Hyperbolic Hausdorff distance for medial axis transformation. Graph. Models 63, 369-384 (2001)

36. Choi, S.W., Seidel, H.-P.: Linear one-sided stability of MAT for weakly injective domain. J. Math. Imaging Vis. 17(3), 237-247 (2002)

37. Choi, S.W., Lee, S.-W.: Stability analysis of medial axis transform. In Proc. 15th ICPR Barcelona, Spain, vol. 3, pp. 139-142 (2000)

38. Christoffle, E.B.: Sul problema della tempurature stazonaire e la rappresetazione di una data superficie. Ann. Mat. Pura Appl. Ser. II, pp. 89-103 (1867) 
39. Cipra, B.: The best of the 20th century: Editors name top 10 algorithms. SIAM News 33(4), 1 (2000)

40. Cooley, J.W., Tukey, J.W.: An algorithm for the machine calculation of complex Fourier series. Math. Comput. 19, 297-301 (1965)

41. Culver, T., Keyser, J., Manocha, D.: Accurate computation of the medial axis of a polyhedron. In: Proceedings of the Fifth ACM Symposium on Solid Modeling and Applications, June 8-11, 1999, Ann Arbor, MI, USA, pp. 179-190 (1999)

42. Daripa, P.: A fast algorithm to solve nonhomogeneous Cauchy-Riemann equations in the complex plane. SIAM J. Sci. Stat. Comput. 13(6), 1418-1432 (1992)

43. Daripa, P.: A fast algorithm to solve the Beltrami equation with applications to quasiconformal mappings. J. Comput. Phys. 106(2), 355-365 (1993)

44. Daripa, P., Mashat, D.: An efficient and novel numerical method for quasiconformal mappings of doubly connected domains. Numer. Algorithms 18(2), 159-175 (1998)

45. Davis, R.T.: Numerical methods for coordinate generation based on Schwarz-Christoffel transformations. In: 4th AIAA Comput. Fluid Dynamics Conf., Williamsburg, VA, pp. 1-15 (1979)

46. DeLillo, T.K.: The accuracy of numerical conformal mapping methods: a survey of examples and results. SIAM J. Numer. Anal. 31(3), 788-812 (1994)

47. Dirichlet, G.L.: Über die Reduktion der positiven quadratischen Formen mit drei unbestimmten ganzen Zahlen. J. Reine Angew. Math. 40, 209-227 (1850)

48. Driscoll, T.A., Trefethen, L.N.: Schwarz-Christoffel Mapping. Cambridge Monographs on Applied and Computational Mathematics, vol. 8. Cambridge University Press, Cambridge (2002)

49. Driscoll, T.A., Vavasis, S.A.: Numerical conformal mapping using cross-ratios and Delaunay triangulation. SIAM J. Sci. Comput. 19(6), 1783-1803 (1998) (electronic),

50. Duan, H.B., Rees, E.: The existence of bitangent spheres. Proc. R. Soc. Edinb. A 111(1-2), 85-87 (1989)

51. Dutt, A., Gu, M., Rokhlin, V.: Fast algorithms for polynomial interpolation, integration, and differentiation. SIAM J. Numer. Anal. 33(5), 1689-1711 (1996)

52. Epstein, D.B.A., Marden, A.: Convex hulls in hyperbolic space, a theorem of Sullivan, and measured pleated surfaces. In: Analytical and Geometric Aspects of Hyperbolic Space, Coventry/Durham, 1984. London Math. Soc. Lecture Note Ser., vol. 111, pp. 113-253. Cambridge Univ. Press, Cambridge (1987)

53. Epstein, D.B.A., Marden, A., Markovic, V.: Quasiconformal homeomorphisms and the convex hull boundary. Ann. Math. (2) 159(1), 305-336 (2004)

54. Epstein, D.B.A., Marden, A., Markovic, V.: Complex earthquakes and deformations of the unit disk. J. Differ. Geom. 73(1), 119-166 (2006)

55. Epstein, D.B.A., Marden, A., Markovic, V.: Convex regions in the plane and their domes. Proc. Lond. Math. Soc. (3) 92(3), 624-654 (2006)

56. Epstein, D.B.A., Markovic, V.: The logarithmic spiral: a counterexample to the $K=2$ conjecture. Ann. Math. (2) 161(2), 925-957 (2005)

57. Erdős, P.: On the Hausdorff dimension of some sets in Euclidean space. Bull. Am. Math. Soc. 52, 107-109 (1946)

58. Evans, G., Middleditch, A., Miles, N.: Stable computation of the $2 \mathrm{D}$ medial axis transform. Int. J. Comput. Geom. Appl. 8(5-6), 577-598 (1998)

59. Evans, W.D., Harris, D.J.: Sobolev embeddings for generalized ridged domains. Proc. Lond. Math. Soc. (3) 54(1), 141-175 (1987)

60. Fortune, S.: Voronor̆ diagrams and Delaunay triangulations. In: Computing in Euclidean Geometry. Lecture Notes Ser. Comput, vol. 1, pp. 193-233. World Scientific, River Edge (1992)

61. Fortune, S.: Voronoi diagrams and Delaunay triangulations. In: Handbook of Discrete and Computational Geometry. CRC Press Ser. Discrete Math. Appl., pp. 377-388. CRC, Boca Raton (1997)

62. Fremlin, D.H.: Skeletons and central sets. Proc. Lond. Math. Soc. (3) 74(3), 701-720 (1997)

63. Gaier, D.: Konstruktive Methoden der konformen Abbildung. Springer Tracts in Natural Philosophy, vol. 3. Springer, Berlin (1964)

64. Garnett, J.B.: Bounded Analytic Functions. Pure and Applied Mathematics, vol. 96. Academic Press [Harcourt Brace Jovanovich Publishers], New York (1981)

65. Gaudeau, C., Boiron, M., Thouvenot, J.: Squelettisation et anamorphose dans l'étude de la dynamique des déformations des structures: application à l'analyse de la motricité gastrique. In: Recognition of Shapes and Artificial Intelligence (Second AFCET-IRIA Cong., Toulouse, 1979), vol. III, pp. 57-63. IRIA, Rocquencourt (1979) (in French)

66. Gehring, F.W.: The definitions and exceptional sets for quasiconformal mappings. Ann. Acad. Sci. Fenn. Ser. A I 281, 28 (1960) 
67. Gehring, F.W.: Symmetrization of rings in space. Trans. Am. Math. Soc. 101, 499-519 (1961)

68. Giblin, P.: Symmetry sets and medial axes in two and three dimensions. In: The Mathematics of Surfaces, IX, Cambridge, 2000, pp. 306-321. Springer, London (2000)

69. Giblin, P.J., O'Shea, D.B.: The bitangent sphere problem. Am. Math. Mon. 97(1), 5-23 (1990)

70. Greengard, L., Rokhlin, V.: A fast algorithm for particle simulations. J. Comput. Phys. 73(2), 325348 (1987)

71. Gursoy, H.N., Patrikalakis, N.M.: Automated interrogation and adaptive subdivision of shape using medial axis transform. Adv. Eng. Softw. Workstations 13(5/6), 287-302 (1991)

72. He, Z.-X., Schramm, O.: On the convergence of circle packings to the Riemann map. Invent. Math. 125(2), 285-305 (1996)

73. He, Z.-X., Schramm, O.: The $C^{\infty}$-convergence of hexagonal disk packings to the Riemann map. Acta Math. 180(2), 219-245 (1998)

74. Heinonen, J., Koskela, P.: Quasiconformal maps in metric spaces with controlled geometry. Acta Math. 181(1), 1-61 (1998)

75. Henrici, P.: Applied and Computational Complex Analysis. Pure and Applied Mathematics, vol. 3. Wiley, New York (1986). Discrete Fourier analysis-Cauchy integrals-construction of conformal maps - univalent functions, A Wiley-Interscience Publication

76. Hertling, P.: An effective Riemann mapping theorem. Theoret. Comput. Sci. 219(1-2), 225-265 (1999). Computability and complexity in analysis (Castle Dagstuhl, 1997)

77. Hoffmann, C.M.: Computer vision, descriptive geometry and classical mechanics. In: Computer Graphics and Mathematics. Eurographics Series, pp. 229-244. Springer, Berlin (1992)

78. Hoffmann, C.M., Dutta, D.: On the skeleton of simple CSG objects. Trans. ASME 115, 87-94 (1993)

79. Holopainen, I.: Rough isometries and $p$-harmonic functions with finite Dirichlet integral. Rev. Mat. Iberoam. 10(1), 143-176 (1994)

80. Hörmander, L.: The Analysis of Linear Partial Differential Operators. I. Grundlehren der Mathematischen Wissenschaften [Fundamental Principles of Mathematical Sciences], vol. 256, 2nd edn. Springer, Berlin (1990). Distribution theory and Fourier analysis

81. Howell, L.H.: Numerical conformal mapping of circular arc polygons. J. Comput. Appl. Math. 46(1-2), 7-28 (1993)

82. Hu, C.: Algorithm 785: a software package for computing Schwarz-Christoffel conformal transformation for doubly connected polygonal regions. ACM Trans. Math. Softw. 24(3), 317-333 (1998)

83. Ivanov, V.I., Trubetskov, M.K.: Handbook of Conformal Mapping with Computer-Aided Visualization. CRC Press, Boca Raton (1995). With 1 IBM-PC floppy disk (5.25 inch; HD)

84. Jinkerson, R.A., Abrams, S.L., Bardis, L., Chryssostomidis, C., Clement, A., Patrikalakis, N.M., Wolter, F.E.: Inspection and feature extraction of marine propellers. J. Ship Product. 9(2), 88-106 (1993)

85. Karatsuba, A., Ofman, Yu.: Multiplication of many-digital numbers by automatic computers. Dokl. Akad. Nauk SSSR 145, 293-294 (1962)

86. Klein, R., Lingas, A.: A linear-time randomized algorithm for the bounded Voronoi diagram of a simple polygon. Int. J. Comput. Geom. Appl. 6(3), 263-278 (1996). ACM Symposium on Computational Geometry (San Diego, CA, 1993)

87. Kythe, P.K.: Computational Conformal Mapping. Birkhäuser, Boston (1998)

88. Lee, D.-T.: The medial axis transform of a planar shape. IEEE Trans. Pattern Anal. Mach. Intell. 4(4), 363-369 (1982)

89. Lee, D.T., Drysdale, R.L. III: Generalization of Voronoŭ diagrams in the plane. SIAM J. Comput. 10(1), 73-87 (1981)

90. Lee, Y.-H., Horng, S.-J.: The equivalence of the chessboard distance transform and the medial axis transform. Int. J. Comput. Math. 65(3-4), 165-177 (1997)

91. Maekawa, T., Patrikalakis, N.M.: Computation of singularities and intersections of offsets of planar curves. Comput. Aided Geom. Design 10(5), 407-429 (1993)

92. Maekawa, T., Patrikalakis, N.M.: Interrogation of differential geometry properties for design and manufacture. Vis. Comput. 10(4), 216-237 (1994)

93. Marshall, D.E., Rohde, S.: Convergence of a variant of the zipper algorithm for conformal mapping. SIAM J. Numer. Anal. 45(6), 2577-2609 (2007) (electronic)

94. Milman, D.: The central function of the boundary of a domain and its differentiable properties. J. Geom. 14(2), 182-202 (1980)

95. Milman, D., Waksman, Z.: On topological properties of the central set of a bounded domain in $\mathbf{R}^{m}$. J. Geom. 15(1), 1-7 (1981) 
96. Mohar, B.: A polynomial time circle packing algorithm. Discrete Math. 117(1-3), 257-263 (1993)

97. Mohar, B.: Circle packings of maps in polynomial time. Eur. J. Combin. 18(7), 785-805 (1997)

98. Nehari, Z.: Conformal Mapping. Dover, New York (1975). Reprinting of the 1952 edition

99. O’Donnell, S.T., Rokhlin, V.: A fast algorithm for the numerical evaluation of conformal mappings. SIAM J. Sci. Stat. Comput. 10(3), 475-487 (1989)

100. O'Rourke, J.: Computational Geometry in C, 2nd edn. Cambridge University Press, Cambridge (1998)

101. Papamichael, N., Saff, E.B. (eds.): Computational complex analysis. J. Comput. Appl. Math. 46(1-2) (1993)

102. Patrikalakis, N.M., Maekawa, T.: Shape Interrogation for Computer Aided Design and Manufacturing. Springer, Berlin (2002)

103. Pottmann, H., Wallner, J.: Computational Line Geometry. Mathematics+Visualization. Springer, Berlin (2001)

104. Preparata, F.P.: The medial axis of a simple polygon. In: Mathematical Foundations of Computer Science, Proc. Sixth Sympos., Tatranská Lomnica, 1977. Lecture Notes in Comput. Sci., vol. 53, pp. 443-450. Springer, Berlin (1977)

105. Preparata, F.P., Shamos, M.I.: Computational Geometry. Texts and Monographs in Computer Science. Springer, New York (1985). An introduction

106. Rajan, V.T.: Optimality of the Delaunay triangulation in $\mathbf{R}^{d}$. Discrete Comput. Geom. 12(2), 189202 (1994)

107. Reif, J.H.: Approximate complex polynomial evaluation in near constant work per point. SIAM J. Comput. 28(6), 2059-2089 (1999) (electronic)

108. Rodin, B., Sullivan, D.: The convergence of circle packings to the Riemann mapping. J. Differ. Geom. 26(2), 349-360 (1987)

109. Schwarz, H.A.: Conforme Abbildung der Oberfläche eines Tetraeders auf die Oberfläche einer Kugel. J. Reine Ange. Math., pp. 121-136, 1869. Also in collected works, Gesammelte Mathematische Abhandlungen, pp. 84-101. Springer, Berlin (1890)

110. Schwarz, H.A.: Gesammelte Mathematische Abhandlungen. Springer, Berlin (1890)

111. Shamos, M.I., Hoey, D.: Closest-point problems. In: 16th Annual Symposium on Foundations of Computer Science (Berkeley, CA, 1975), pp. 151-162. IEEE Computer Society, Long Beach (1975)

112. Sherbrooke, E.C., Patrikalakis, N.M., Brisson, E.: Computation of the medial axis transform of 3-d. In: Symposium on Solid Modeling and Applications, pp. 187-200 (1995)

113. Sherbrooke, E.C., Patrikalakis, N.M., Brisson, E.: An algorithm for the medial axis transform of $3 \mathrm{~d}$ polyhedral solids. IEEE Trans. Vis. Comput. Graph. 2(1), 44-61 (1996)

114. Sherbrooke, E.C., Patrikalakis, N.M., Wolter, F.-E.: Differential and topological properties of medial axis transforms. CVGIP: Graph. Model Image Process. 58(6), 574-592 (1996)

115. Smith, W.D.: Accurate circle configurations and numerical conformal mapping in polynomial time. Unpublished technical memorandum, NEC Research Institute, Princeton, NJ (1991)

116. Stephenson, K.: Circlepack. Software available from http://www.math.utk.edu/ kens/

117. Stephenson, K.: The approximation of conformal structures via circle packing. In: Computational Methods and Function Theory 1997 (Nicosia). Ser. Approx. Decompos., vol. 11, pp. 551-582. World Scientific, River Edge (1999)

118. Stephenson, K.: Circle packing and discrete analytic function theory. In: Handbook of Complex Analysis: Geometric Function Theory, vol. 1, pp. 333-370. North-Holland, Amsterdam (2002)

119. Stephenson, K.: Circle packing: a mathematical tale. Not. Am. Math. Soc. 50(11), 1376-1388 (2003)

120. Strebel, K.: On the existence of extremal Teichmueller mappings. J. Anal. Math. 30, 464-480 (1976)

121. Sullivan, D.: Travaux de Thurston sur les groupes quasi-fuchsiens et les variétés hyperboliques de dimension 3 fibrées sur $S^{1}$. In: Bourbaki Seminar, vol. 1979/80, pp. 196-214. Springer, Berlin (1981)

122. Tang, Z.: Fast Transformations Based on Structured Matrices with Applications to the Fast Multipole Method. PhD thesis, University of Maryland, College Park, Maryland (2004)

123. Thom, R.: Sur le cut-locus d'une variété plongée. J. Differ. Geom. 6, 577-586 (1972). Collection of articles dedicated to S.S. Chern and D.C. Spencer on their sixtieth birthdays

124. Thurston, W.P.: The Geometry and Topology of 3-Manifolds. The Geometry Center, University of Minnesota (1979)

125. Trefethen, L.N. (ed.): Numerical Conformal Mapping. North-Holland, Amsterdam (1986). Reprint of J. Comput. Appl. Math. 14(1-2) (1986)

126. Trefethen, L.N., Driscoll, T.A.: Schwarz-Christoffel mapping in the computer era. In: Proceedings of the International Congress of Mathematicians, vol. III, pp. 533-542 Berlin (1998) (electronic) 
127. van der Hoeven, J.: Fast evaluation of holonomic functions. Theoret. Comput. Sci. 210(1), 199-215 (1999)

128. van der Hoeven, J.: Relax, but don't be too lazy. J. Symb. Comput. 34(6), 479-542 (2002)

129. von Koppenfels, W., Stallmann, F.: Praxis der konformen Abbildung. Die Grundlehren der mathematischen Wissenschaften, vol. 100. Springer, Berlin (1959)

130. Voronoi, G.M.: Nouvelles applications des paramètres continus à la théorie des formes quadratiques. recherches sur les parallélloèdres primitifs. J. Reine Angew. Math. 134, 198-287 (1908)

131. Wang, J.: Medial axis and optimal locations for min-max sphere packing. J. Comput. Optim. 4(4), 487-503 (2000)

132. Wegmann, R.: Methods for numerical conformal mapping. In: Handbook of Complex Analysis: Geometric Function Theory, vol. 2, pp. 351-477. Elsevier, Amsterdam (2005)

133. Woess, W.: Random Walks on Infinite Graphs and Groups. Cambridge Tracts in Mathematics, vol. 138. Cambridge University Press, Cambridge (2000)

134. Wolter, E.-F.: Cut locus and the medial axis in global shape interrogation and representation. MIT, Dept. of Ocean Engineering, Design Laboratory Memorandum 92-2 (1993)

135. Wu, Q.J.: Sphere packing using morphological analysis. In: Discrete Mathematical Problems with Medical Applications, New Brunswick, NJ, 1999. DIMACS Ser. Discrete Math. Theoret. Comput. Sci., vol. 55, pp. 45-54. Am. Math. Soc., Providence (2000)

136. Yao, C., Rokne, J.G.: A straightforward algorithm for computing the medial axis of a simple polygon. Int. J. Comput. Math 39, 51-60 (1991)

137. Yap, C.-K.: An $O(n \log n)$ algorithm for the Voronor diagram of a set of simple curve segments. Discrete Comput. Geom. 2(4), 365-393 (1987)

138. Zhou, Q.: Computable real-valued functions on recursive open and closed subsets of Euclidean space. Math. Logic Q. 42(3), 379-409 (1996) 